PHOSPHATE AS AN INDICATOR OF OCCUPATIONAL INTENSITY 


\title{
PHOSPHATE AS AN INDICATOR OF OCCUPATIONAL INTENSITY AT SHELL MIDDEN SITES ON THE CENTRAL COAST OF BRITISH COLUMBIA
}

By KARI J. CARTER, B.A.

\author{
A Thesis \\ Submitted to the Department of Anthropology \\ and the School of Graduate Studies \\ in Partial Fulfillment of the Requirements \\ for the Degree of \\ Master of Arts
}

McMaster University

(C) Copyright by Kari Jane Carter, September 2016 
McMaster University MASTER OF ARTS (2016) Hamilton, Ontario (Anthropology)

TITLE:

Phosphate as an indicator of occupational intensity at shell midden sites on the central coast of British Columbia

AUTHOR:

Kari J. Carter

SUPERVISOR:

Dr. Aubrey Cannon

NUMBER OF PAGES: vii, 107 


\begin{abstract}
This thesis explores phosphate as an indicator of occupational intensity (i.e. as a function of scale and length of occupation) at shell midden sites on the central coast of British Columbia. Despite the prevalence of shell middens in coastal environments world-wide and the long history of elemental analysis in archaeology, shell middens are not routinely investigated for their chemical content. Ongoing research on the British Columbia central coast has shown clear associations between fish bone densities (NISP/L) and site area $\left(\mathrm{m}^{2}\right)$, which have proven useful for characterizing variability among settlements in the region. This provided the opportunity and essential basis for investigating phosphate. Due to its general abundance, persistence, and established association with human activity, phosphate was expected to reflect previously inferred patterns in occupational intensity, which were based on fish bone density and site area data. Results show clear relationships between phosphate values, fish bone densities, and site area, which speaks to the utility of phosphate as an independent indicator of the relative intensity of residential activity among sites.
\end{abstract}




\section{ACKNOWLEDGEMENTS}

I would like to express my thanks first to my supervisor, Dr. Aubrey Cannon, for constantly challenging me. His patience, kindness, and encouragement go above and beyond. I also wish to thank Dr. Andy Roddick for being on my committee and providing critical, helpful, and thoughtful feedback at many points throughout this process. Thanks also to Dr. Ed Reinhardt for being my third examiner, for providing helpful comments on this thesis, and guiding me through XRF analysis. I am grateful to have had the opportunity to learn from all of you.

I also owe my sincere thanks to Dr. Paul Prince for his continued mentorship and for setting me off on the right track.

I am also grateful to Dr. Andrew Martindale for permission to use the Prince Rupert Harbour samples.

Thanks to my dear friend Mary Lynn Tobiasz, for making the beautiful maps that are included in this thesis, but also for her constant support and friendship. I am so glad that moving to Hamilton to get my master's also meant meeting you. Thanks also to Daiana Rivas-Tello, who (among many other things) read multiple drafts of my writing - what would I have done without you? Thank you for being such an amazing support system and a great friend.

Thanks also to Kandace for her advice, friendship, and for trips to the RBG!

Thank you to everyone in the anthropology department at McMaster who are all amazing people and made this experience a fantastic and well-rounded one. I am happy to have been able to spend the last couple of years playing trivia, doing yoga, hiking to waterfalls, playing soccer, going to beaches, attending musicals, picking berries, writing, studying, and eating chocolate with all of you. To those back home in Alberta (and B.C.), thank you for your long-distance support. It means that world to me that you all remain my friends.

I wish especially to thank my family for supporting me on this most recent adventure and all others. Mom, Dad and Jordan, your love and care mean more to me than I can express. Thanks for encouraging me to gallivant across the country even though you'd rather I stay home.

I feel fortunate to have received financial support through a Canadian Graduate Scholarship (CGS-M) and an Ontario Graduate Fellowship, and from the Department of Anthropology and the School of Graduate Studies at McMaster. This research was also supported by a research grant to Aubrey Cannon from the Social Sciences and Humanities Research Council of Canada (SSHRC).

My last bit of thanks goes to David - there are no words. Your support means more to me than you could possibly know. 


\section{Table of Contents}

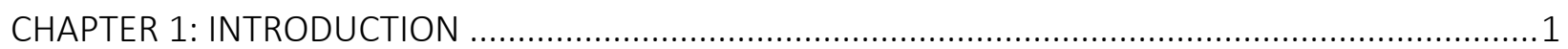

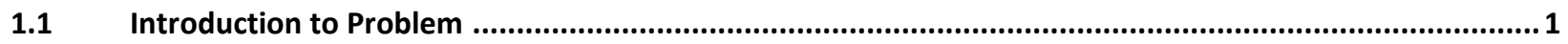

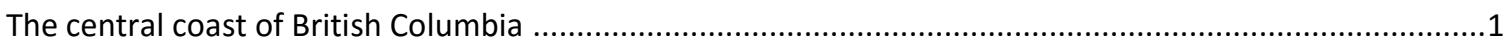

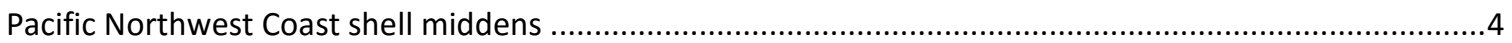

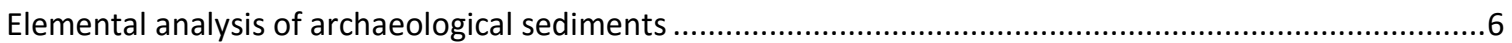

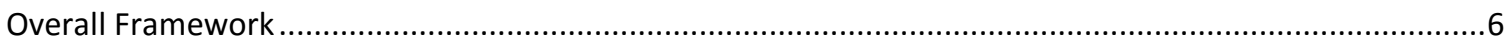

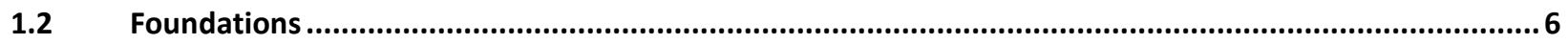

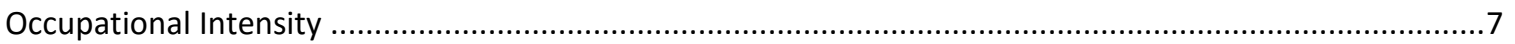

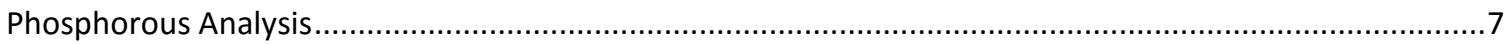

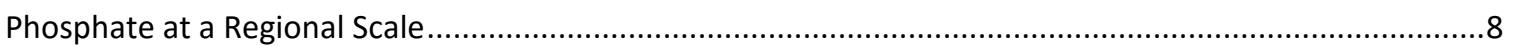

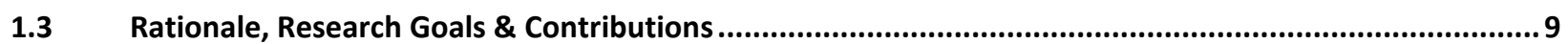

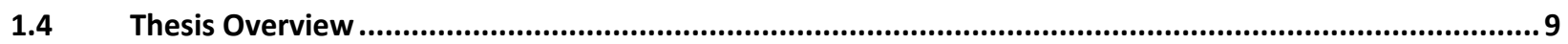

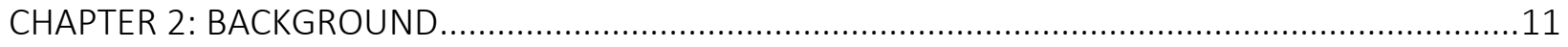

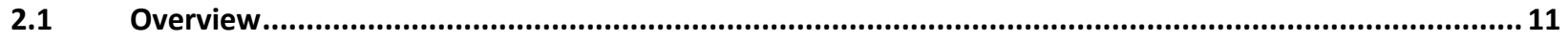

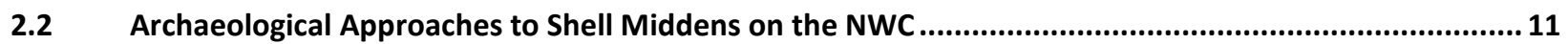

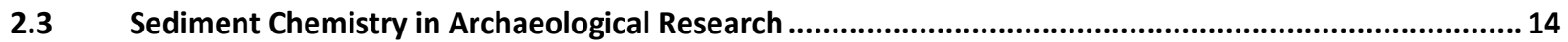

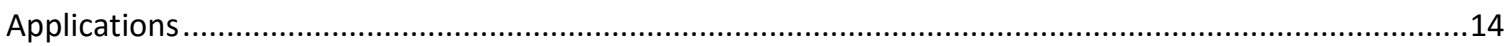

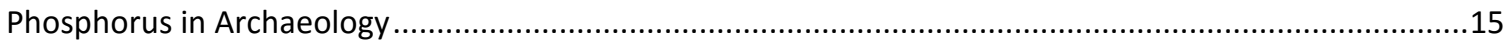

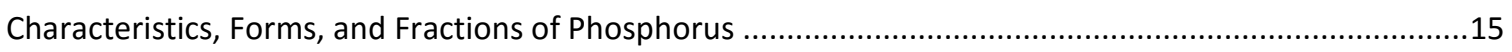

Measuring Phosphorus in Archaeological Sediments ..........................................................................19

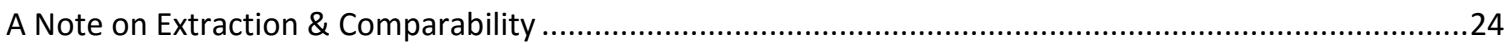

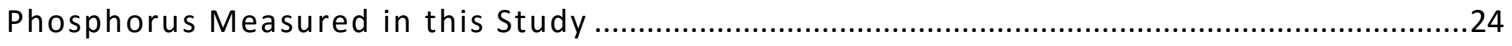

2.4 Factors Influencing the Chemical Composition of Archaeological Sediments .........................................26

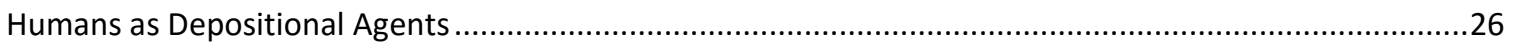

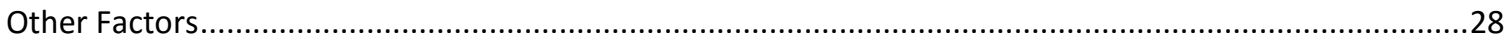

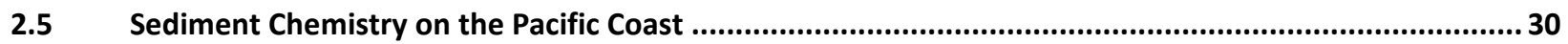

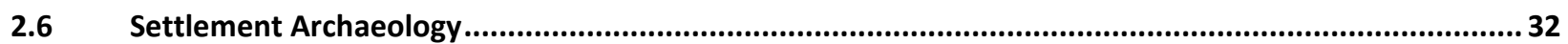

Some Themes

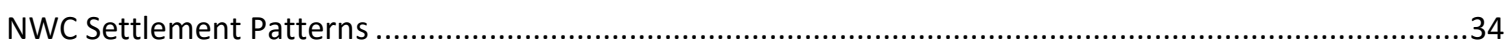

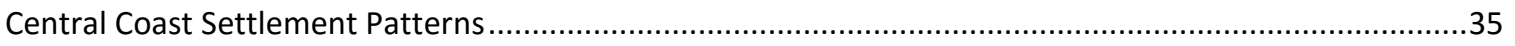

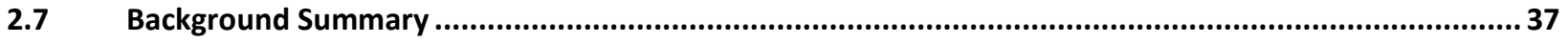

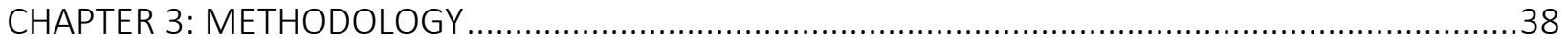

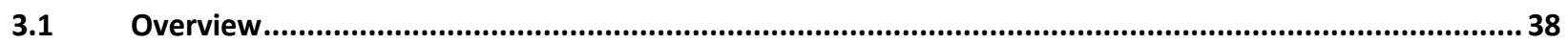

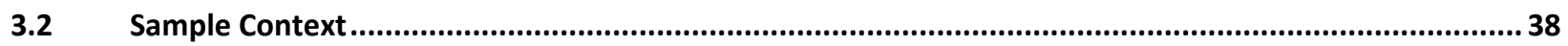

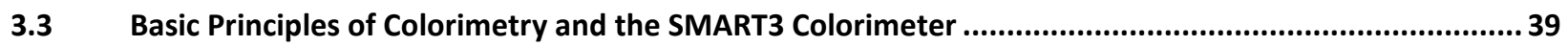

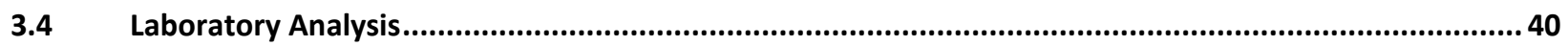

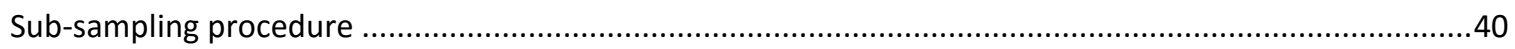

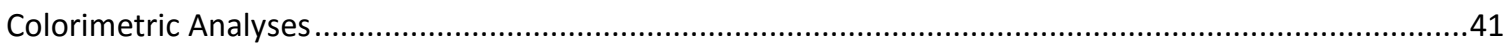

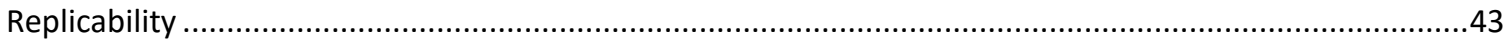

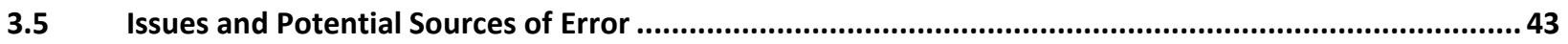

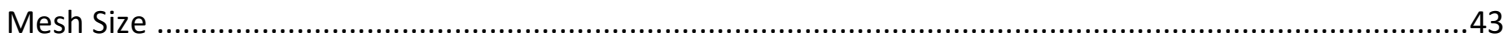

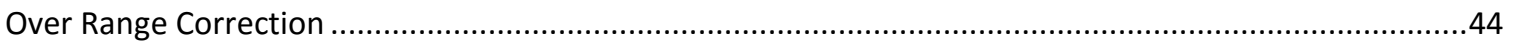

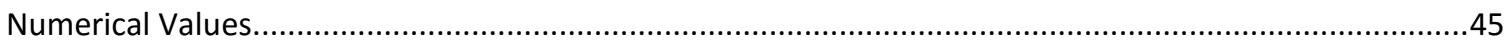




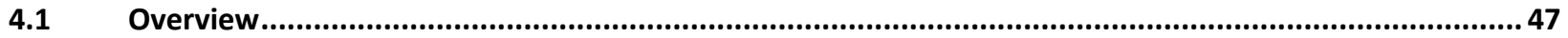

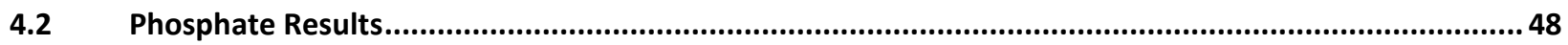

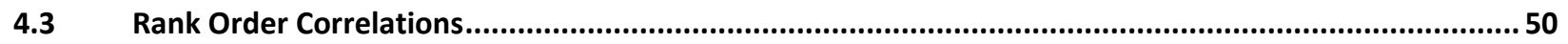

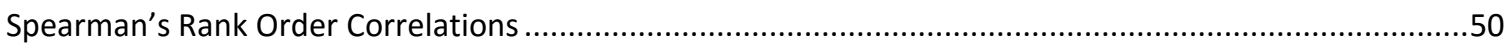

4.4 Inferences \& Some Meaningful Comparisons ...........................................................................53

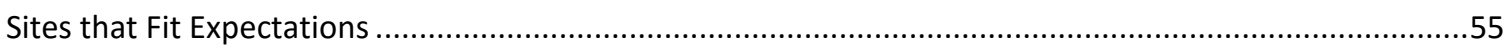

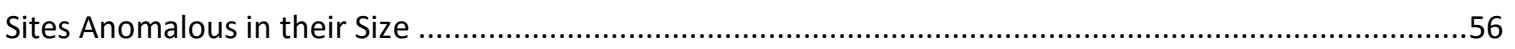

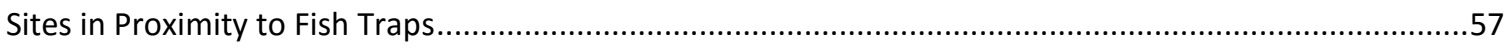

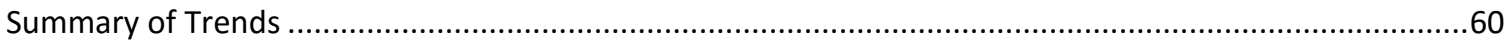

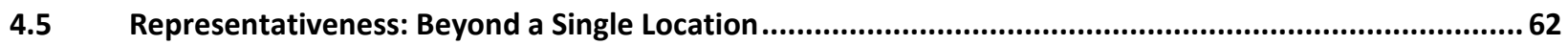

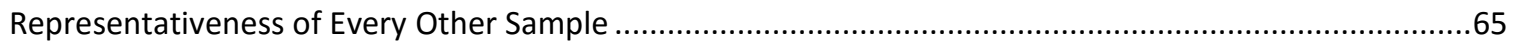

4.6 Generalizability: Beyond British Columbia's Central Coast ..........................................................65

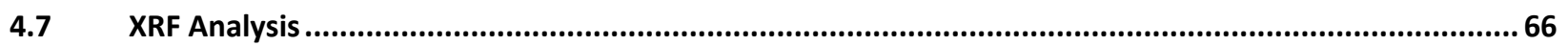

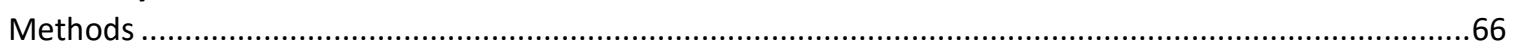

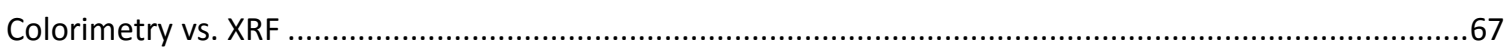

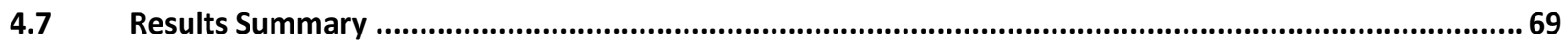

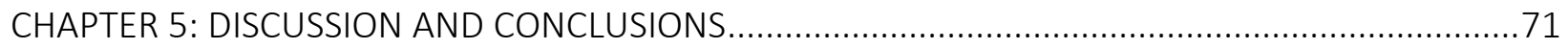

5.1 Within a Settlement Narrative for the British Columbian Central Coast .............................................. 71

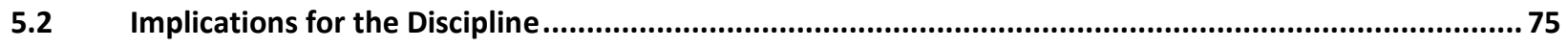

What Phosphate means for Shell Midden Archaeology.........................................................................75

What Phosphate Analysis of Shell Midden Sediments means for Archaeological Soil and Sediment

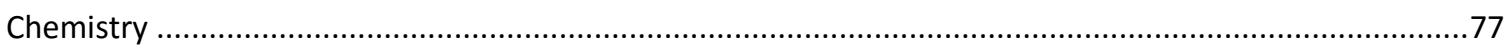

How Phosphate speaks to Long-term Histories and Regional Archaeologies ...........................................78

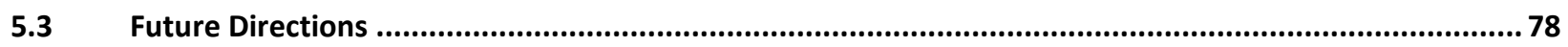

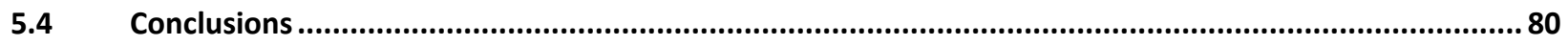

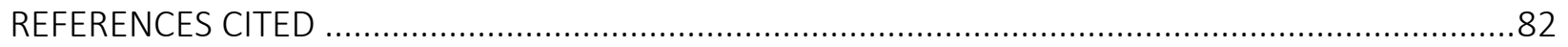

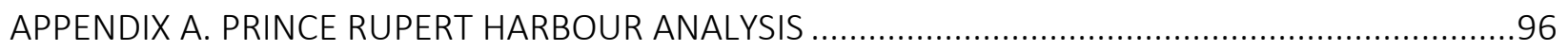

APPENDIX B. LAMOTTE SMART3 COLORIMETERS PUT TO THE TEST ........................................98

APPENDIX C. MAJOR SHELL MIDDEN CONSTITUENTS: HOW MUCH PHOSPHATE? .....................99

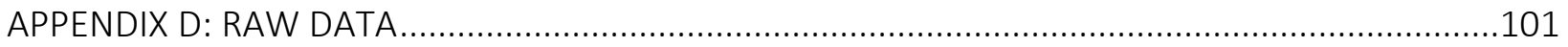




\title{
List of Figures
}

FIGURE 1.1. Location of sites tested in this study within the context of all sites investigated by Cannon ..................2

FIGURE 1.2. Exposed stratigraphy at EjSw-1 (Cockmi) as an example of complex shell midden deposition .................

FIGURE 2.1. Pathways and interactions of the different forms and fractions of soil $P$..........................................18

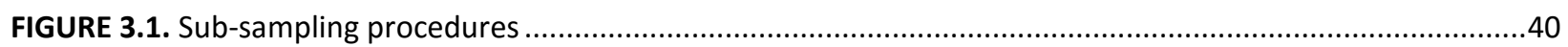

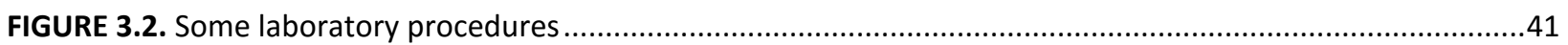

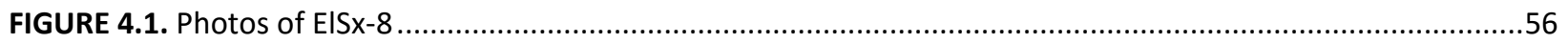

FIGURE 4.2. Showing variability in shell content at twelve central coast sites ....................................................59

FIGURE 4.3. Showing the somewhat linear relationship between phosphate, fish bone density and site area, and how the anomalous sites fall out of the pattern in particular ways. ............................................................6

FIGURE 4.4. Map showing location of the PRH in relation to Namu and the central coast .....................................66

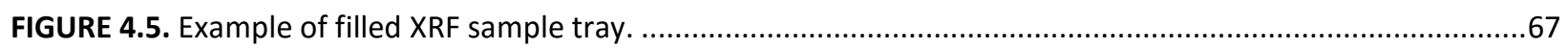

FIGURE 4.6. Showing comparisons between fish bone density, phosphate (colorimeter) and phosphorus (XRF) at

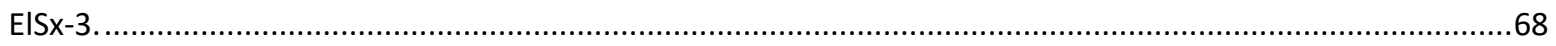

\section{List of Tables}

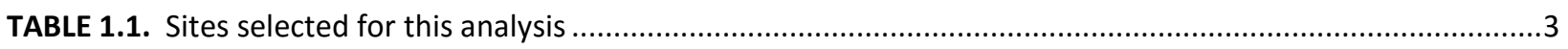

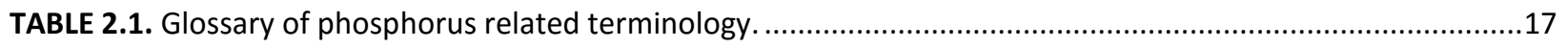

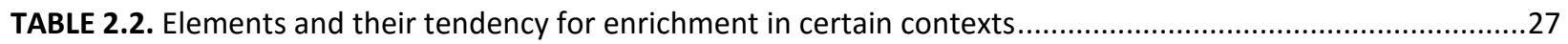

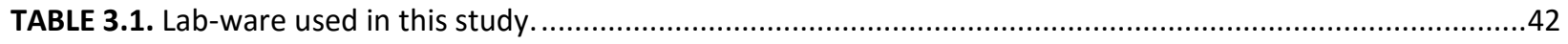

TABLE 4.1. List of all sites tested in this study from highest to lowest phosphate content ....................................48

TABLE 4.2. Differences between phosphate and fish bone density rankings ........................................................51

TABLE 4.3. Differences between phosphate and site area rankings .......................................................................52

TABLE 4.4. Sites tested in this study grouped based on FBD categories defined by Cannon ...................................54

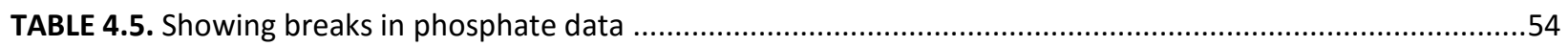

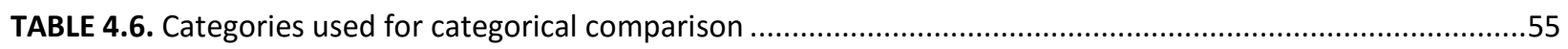

TABLE 4.7. Rank orderings for phosphate, fish bone density, and site area, and their sums. Showing sites on a scale

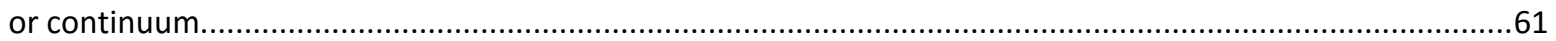

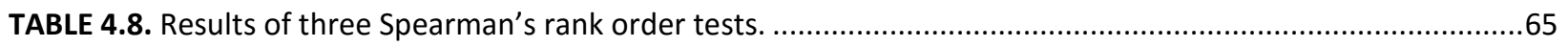

\section{List of Abbreviations}

\author{
FBD - fish bone density \\ NISP - number of identified specimens \\ NWC - Northwest Coast \\ PRH - Prince Rupert Harbour
}




\section{M.A. Thesis - K. Carter; McMaster University - Anthropology}

\section{Chapter 1: Introduction}

\subsection{Introduction to Problem}

This thesis explores the efficacy of phosphate analysis for indicating variability in occupational intensity (i.e. as a function of scale and length of occupation) at shell midden sites located on the central coast of British Columbia, within the traditional territories of the Heiltsuk and Wuikinuxv Nations. To explore this application, I compared phosphate values obtained from fine-fraction matrix with fish bone densities (NISP/L). Fish bone densities, primarily in combination with site area, but also a number of other archaeological data, have been linked to varying intensities of occupation among settlements in the region (Cannon 2013a). My primary goal was to assess the value of phosphate as an independent indicator of these previously inferred patterns.

\section{The central coast of British Columbia}

This thesis research contributes the perspective of phosphate analysis to archaeological investigations of shell middens and their formation, and more specifically to a large-scale research project that integrates the 11,000-year-old archaeological village at Namu within a broader regional context (Cannon 2000a, 2000b, 2002, 2013a). Past archaeological investigations have documented the history and focus of the Namu site, and highlighted long-term marine subsistence strategies (Cannon 1991, 1998a; Carlson 1979, 1996). Cannon's on-going project has developed an additional understanding of the history of settlement organization in the region through a field strategy based on coring and auger sampling of multiple sites, rather than fullscale excavation of single sites (Cannon 1998a, 1997, 2000a, 2000b, 2013b).

Cannon tested a number of sites over the course of six field seasons in the vicinity of Namu and in nearby Rivers Inlet (Cannon 1997, 1998b, 2013b) (see Figure 1.1). These sites were chosen with the goal of sampling a range of site types in different locations, including the exposed outer coast and the relatively protected inner coast. Sites in Rivers Inlet were sampled in order to gain a better understanding of that area's settlement history, especially compared with the range of variability evident around Namu and the outer coast (Cannon 2013b). So far, thirty sites have been investigated. 


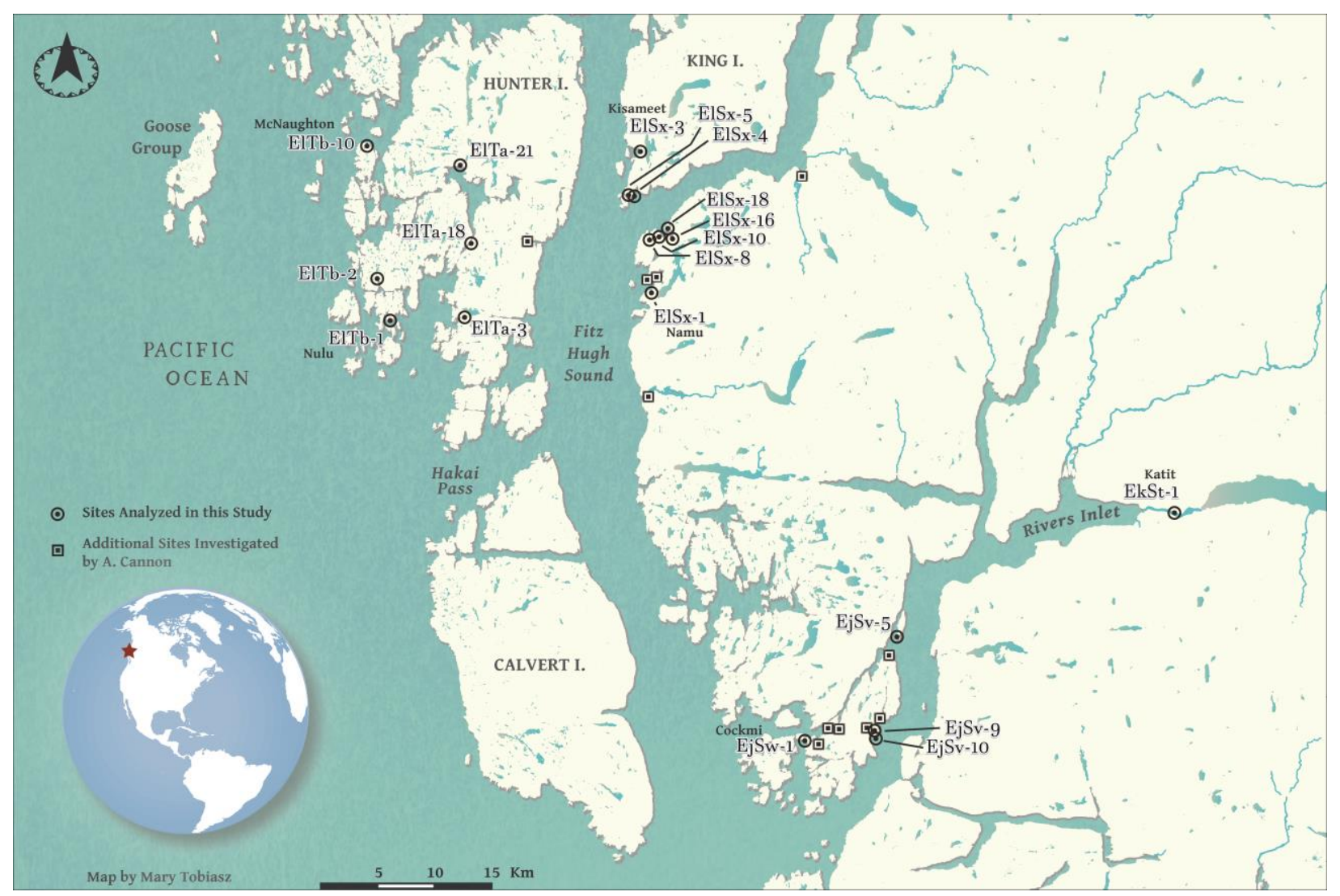

FIGURE 1.1. Location of sites tested in this study within the context of all sites investigated by Cannon (2000a, 2000b, 2002, 2013a). Map by Mary Lynn Tobiasz.

Intensive analysis of small samples from these sites - which has included assessments of shell fragmentation, characterization of red ochre, and identification of faunal material, parasite eggs, and seeds introduced by humans - has addressed archaeological questions surrounding health (Bathurst 2005a), distribution of red ochre (MacDonald 2008; MacDonald et al. 2011), patterns of shellfish harvesting and site seasonality (Burchell et al. 2013a, 2013b; Cannon and Burchell 2009, 2016), and fisheries (Cannon 2000a). Taken together, these analyses show wide variation in site content. This, coupled with differences in site area, has been attributed to differences in the intensity of occupation and activity foci at particular places (Cannon 2013a). Specifically, densities of fish bone (NISP standardized per litre of fine fraction $[<2 \mathrm{~mm}]$ matrix) combined with the overall surface area of shell midden sites has shown patterns interpreted as variations in the nature and intensity of site occupation. "Villages occupied for longer periods by a greater number of people exhibited the greater density and variety of fish remains, while sites thought to have been occupied less frequently or for shorter periods by smaller groups exhibited much less density and variety of fish" (Cannon 2013a:25). A range of relatively intense 


\section{M.A. Thesis - K. Carter; McMaster University - Anthropology}

occupations, from sites identified as short-term camps to those considered longer-term residential locations, has been discerned through these relationships, a pattern that Cannon (2013a:25) suggests is a continuum.

This is the pattern I set out to independently evaluate using phosphates. Of the thirty sites investigated by Cannon, I analyzed nineteen (see Table 1.1 and Figure 1.1). These sites represent the range of occupational variability evident on the central coast.

TABLE 1.1. Sites selected for this analysis. Information adapted from Cannon (2013a:24). Fish bone densities were calculated per litre of fine fraction $(<2 \mathrm{~mm})$ matrix.

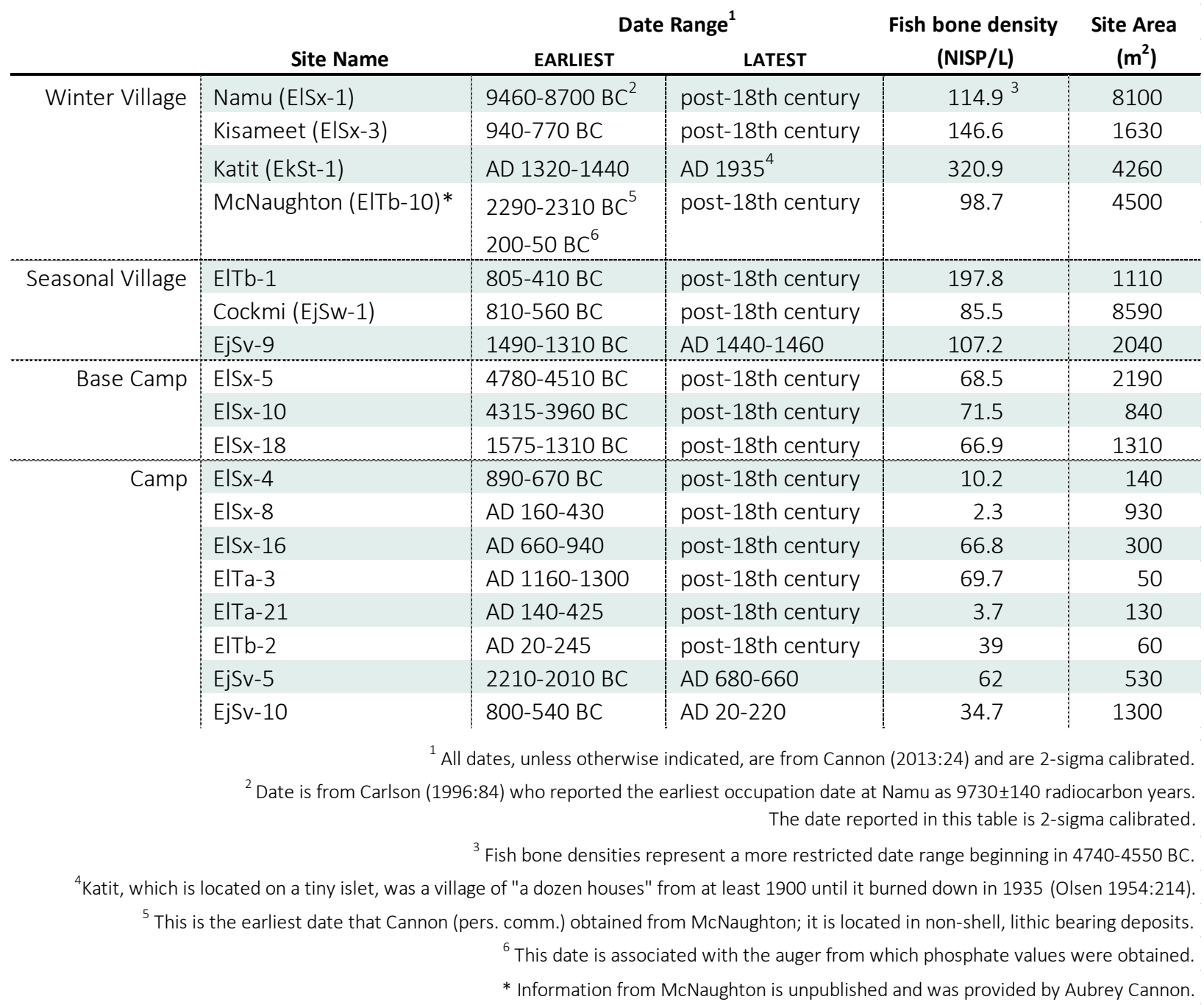


Pacific Northwest Coast shell middens

Cannon's auger sampling strategy and regional program is one approach to the stratigraphically complex structure of Northwest Coast shell middens, which have challenged traditional archaeological methods and prompted the development of innovative strategies to help understand their accumulation (Stein et al. 2003:298). Shell middens on the Pacific Northwest Coast (NWC) consist primarily of cultural materials with a proportion of matrix (i.e. shell and rock) that is much greater than artifact content (Stein et al. 2003; Stein 2008). Both horizontal and vertical discontinuity is prevalent at these sites (see Figure 1.2) (Waselkov 1987:116). Archaeologists must excavate large amounts of material in broad stratigraphic levels or layers to construct chronologies using traditional artifact associations (Hester and Conover 1970:138; Lyman 1991; Stein 2008), which is a situation unique to shell middens. The character of individual middens is also variable in size ${ }^{1}$, form, and distribution

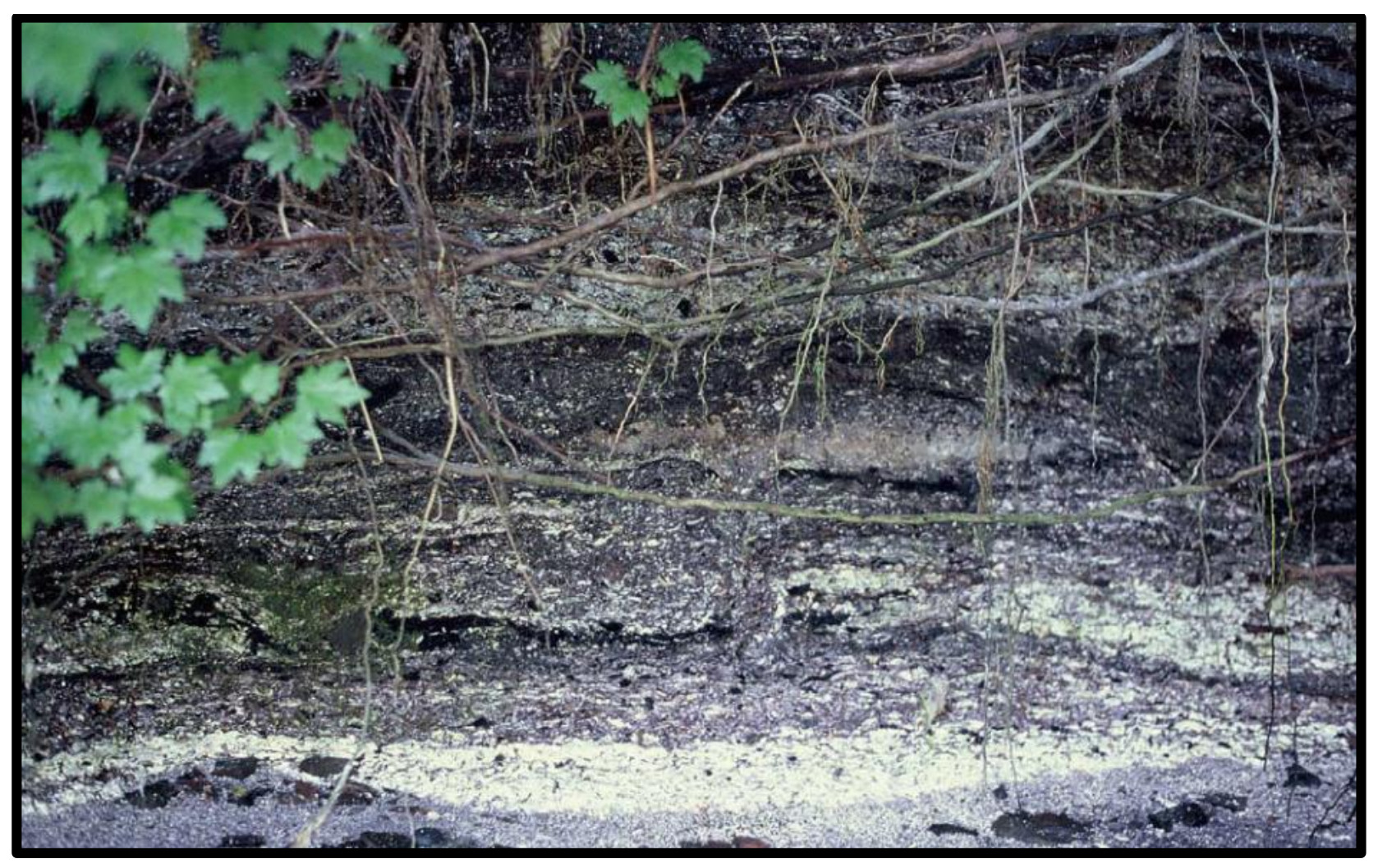

FIGURE 1.2. Exposed stratigraphy at EjSw-1 (Cockmi) as an example of complex shell midden deposition. From Cannon (2013b:11).

\footnotetext{
${ }^{1}$ See Tobiasz (2015:55) for an illustration that shows differences between some small and large shell middens.
} 


\section{M.A. Thesis - K. Carter; McMaster University - Anthropology}

Shell middens are further complicated in their rates of accumulation (Stein et al. 2003). In addition to the complexities that can be physically observed in matrix stratigraphy (see Figure 1.2), sites that seem similar in their overall structures may have been deposited over different amounts of time and at different rates. One very deep shell midden may be the result of longterm, continual occupations, while another very deep shell midden may be the result of multiple, short-term and infrequent occupations. In other words, fast rates of deposition over short amounts of time can produce a shell midden structurally similar to one that was produced very slowly over a long period. Discontinuities resulting from hiatuses in occupation are not discernable through stratigraphic analyses, and even rigorous radiocarbon dating regimes (including 82 radiometric dates for just six sites in one study by Stein and colleagues) are not sufficient for highlighting clear patterns of deposition over time (Stein et al. 2003:301).

Due to the spatially discontinuous nature of shell midden accumulation, it is also inappropriate to assume contemporaneity of deposits at the same level on or below the surface. In contrast to sites where house floors and architectural structures and features are clearly resolved, shell midden deposits on the same level could be relatively contemporaneous (i.e. the result of deposition within the same week, year, or decade), or they could be separated in time by hundreds or thousands of years.

Archaeologists have approached these complexities differently. Some have preferred full excavation of shell middens in arbitrary stratigraphic levels to obtain the information necessary to construct culture-historical sequences (e.g. Percy 1974; Trace 1981). Others have focussed their efforts on detailed analysis of the matrix itself and its components through, for example, intensive full-scale excavation (e.g. Ham 1982), surface exploration (e.g. Dalan et al. 1992), and strategic small-scale, sub-surface investigation (e.g. Acheson 1995; Cannon 2000a). Small-scale sampling in particular allows for regional investigations that would be prohibitively costly and time consuming using traditional methods of site excavation (Cannon 2013a:22). Although archaeologists integrating multiple proxies and conducting detailed matrix analyses endeavour to address different kinds of questions, they all share an interest in using these time-averaged deposits to their fullest potential. My thesis aligns with this history of interest in shell midden content and their depositional complexities, and contributes to the argument in favour of small- 


\section{M.A. Thesis - K. Carter; McMaster University - Anthropology}

scale sampling and intensive analysis (Brewster and Martindale 2011; Cook and Treganza 1947, 1948; Hester and Conover 1970; McKechnie 2013; Stein 1986).

\section{Elemental analysis of archaeological sediments}

Archaeologists employ sediment chemistry to explore on-site spatial patterning, address post-depositional site alterations, determine site extent, detect and analyze activities that may have only left a chemical signature, and distinguish cultural from non-cultural deposition (e.g. Conway 1983; Cook and Heizer 1965; Eidt 1977; Entwistle et al. 2000; Griffith 1980; Knudson and Frink 2010b; Manzanilla and Barba 1990; Middleton 2004; Middleton and Price 1996; Middleton et al. 2010; Moss 1984; Oonk et al. 2009; Parnell et al. 2002; Schlezinger and Howes 2000; Terry et al. 2000). Despite its use for investigating archaeological questions, sediment chemistry has rarely been employed in shell midden contexts.

\section{Overall Framework}

In sum, the framework of this thesis rests essentially on (1) the variability in shell midden site history, form and content evident from Cannon's investigations on the central coast of British Columbia, (2) the depositional and stratigraphic complexities of Pacific Northwest Coast shell middens, which demand alternative approaches to traditional excavation for their regional investigation, and (3) the widely documented human contribution to the elemental composition of archaeological sediments.

The remainder of this chapter presents the conceptual foundations for this thesis, including a working definition of occupational intensity. Following this, I present the specific rationale for this study, and outline the format of the remaining chapters.

\subsection{Foundations}

In this thesis I explore whether phosphate is a good indicator of occupational intensity at shell midden sites by seeing if it reflects patterns previously inferred using other indicators -

namely fish bone densities and site area. This section answers three foundational questions: What is occupational intensity? Why phosphate? And how is phosphate, which is traditionally used to address archaeological questions at a smaller spatial scale, suitable for a regional analysis? 


\section{M.A. Thesis - K. Carter; McMaster University - Anthropology}

\section{Occupational Intensity}

Occupational intensity can be defined as a variable combination of scale (or number of people), duration (or length of time), and intermittency (or frequency) of occupations at a site (Cannon 2013a:22). There is no individual measure for each of these variables. Different numbers of people built up shell middens, but it is impossible to know such demographics. Overall site chronologies can be reconstructed based on radiocarbon dates, but the complexities of shell midden deposits inhibit archaeologists' ability to define the duration of individual occupations that make up a whole site. In a similar way, intermittencies in occupation are largely indistinguishable, so it is impossible to know how often people returned to places. These issues preclude individual measures of these three variables.

Instead, scale, duration, and frequency of occupation come together in variable combinations at different places. These combinations position sites on a continuum of occupational intensity. Although we do not know the exact contributions of each variable to this measure, it can be used to compare the relative intensity of residential activities among sites. A site, such as a small campsite, that was occupied by few people, for a short period, and relatively infrequently would be at the low end of an occupational intensity continuum. In contrast, a site, such as a village, that was occupied by many people, for a long period, and relatively frequently would be on the high end of the scale. Any combination of these three variables can exist in between. Occupational intensity, then, is multi-faceted and can be understood at different scales depending on the resolution of archaeological data. Different combinations of variables result in different intensities of occupation.

\section{Phosphorous Analysis}

Phosphorous is a physically abundant and persistent element that has a long history of use in archaeology (Holliday and Gartner 2007:301). Its nature, cycling and deposition are complicated, but archaeologists have established that many human activities can deposit phosphorus $^{2}$. In particular, hearths (or ash from fires), plant materials, human and animal excreta, bone, kiln areas, fish processing areas, and kitchen areas have been cited as contributors of and areas high in phosphorus (Cook and Heizer 1965; Holliday and Gartner 2007; Knudson and

\footnotetext{
${ }^{2}$ I elaborate on this in Chapter 3: Methods
} 


\section{M.A. Thesis - K. Carter; McMaster University - Anthropology}

Frink 2010b; Misarti et al. 2011; Moss 1984). Phosphorus is therefore a ubiquitously distributed trace of human activity, which is well suited to unbiased recovery in small-scale auger samples.

\section{Phosphate at a Regional Scale}

Archaeological sediment chemistry is largely employed in the horizontal spatial analysis of activity areas (usually on house floors), but I conducted phosphate analysis on the finefraction matrix from auger samples of depositionally complex shell middens. The results are directed toward characterization of site locations in relation to one another. Application of phosphate at this coarse resolution in effect views sites within regions in the same way that activity areas might be viewed within the context of sites or house areas within sites, the scale at which phosphate analysis is more typically applied. In theory, patterns at a site-level that represent the average of multiple accumulated daily activities over a long period could be interpreted as intensively occupied. This is similar to how repeated use of an area for cooking would accumulate and average out as what archaeologists would interpret as a "cooking area". Considered within the spatial context of a house, such use areas provide insight into the way daily life played out in a particular place, usually the home. Considered within the spatial context of a broad geographic and temporal landscape, site-level averages speak to coarser, regional patterns. We get an idea about the intensity and activity foci at particular 'concentrated use areas' that we call sites.

Time perspectivism - which is in part the idea that different scales of analysis provide insights into different kinds of events and processes (e.g. Bailey 2008:13) - has influenced my thinking about phosphates in this way and at this scale of analysis. By considering that "shortlived phenomena require highly resolved measures of time for their observation and study, while larger and more extensive phenomena require and permit a coarser scale of measurement" (Bailey 2007:201), phosphate analysis of complex deposits can be understood in relation to the more extensive phenomenon of regional settlement patterns accumulated or averaged over time, rather than the intra-site activity areas in more temporally constrained archaeological contexts, where it is typically employed. In this way, the chemical analysis of sediments is easily translatable to a regional scale, and has an informative place in NWC shell midden archaeology. 


\section{M.A. Thesis - K. Carter; McMaster University - Anthropology}

\subsection{Rationale, Research Goals \& Contributions}

Despite their prevalence in coastal environments world-wide, archaeologists rarely investigate shell middens for their elemental content. The well-established context that scholars have developed for the central coast of British Columbia provided an opportunity to assess the feasibility and value of investigating chemical signatures in shell midden contexts, while simultaneously adding to an understanding of the area's history using a method that had not yet been applied there.

I was interested in applying the general premise of archaeological sediment chemistry (i.e. that humans chemically alter the soil or sediment) to extend its application more broadly to shell midden sites. Specifically, I was curious about the relationship between elemental signatures and relative occupational intensity, and between elemental signatures and regional activity foci. It has also been pointed out that archaeologists tend to use sediment chemistry to "support conclusions already drawn" (Bethell and Máté 1989:14; Parnell et al. 2001:856). I was further interested in the degree to which an elemental application could provide either additional details that could not be known otherwise, or refine techniques and strategies, following from Parnell et al.'s (2001:856) similar motivations.

The results of phosphate analysis generally conforms to patterns in site use intensity, established on the basis of fish bone density and site area. They also point to some readily explainable anomalous sites, which I turn to in following chapters. This demonstrates the broader utility of this method for investigating shell middens.

\subsection{Thesis Overview}

In Chapter 2, I provide a brief background on the history of archaeological investigations in this region. I give more detail on studies that have focussed on the analysis of shell midden sediments and matrix. I also discuss archaeological investigations of regional settlement patterns more broadly. I discuss methodologies employed in this study in Chapter 3. This includes a discussion of site investigations conducted by Cannon, as well as the sub-sampling and colorimetry procedures I followed in the lab. In Chapter 4, I present the results of this study, highlighting the relationship between phosphate and fish bone densities, and between phosphate and site area. I highlight the trends that are evident among the nineteen sites tested, and discuss 
M.A. Thesis - K. Carter; McMaster University - Anthropology

anomalous results. In Chapter 5, I contextualize my findings within a broader context. It further ties together overarching themes and restates conclusions. 


\section{M.A. Thesis - K. Carter; McMaster University - Anthropology}

\section{Chapter 2: Background}

\subsection{Overview}

In this chapter, I align my research with archaeological investigations of Pacific Northwest Coast shell middens that have focussed on matrix. I then provide background on sediment chemistry in archaeological research broadly before moving into a more detailed discussion of the analysis and interpretation of phosphorus within archaeology. Following this, I highlight pertinent factors that influence soil or sediment chemistry. I focus here on the premise that humans as depositional agents alter soil/sediment chemistry. I highlight archaeological studies that have employed sediment chemistry on the Pacific coast of North America and end with a broader discussion of settlement pattern studies in archaeology.

\subsection{Archaeological Approaches to Shell Middens on the NWC}

Early work on the Pacific Northwest Coast focussed on the detailed analysis of shell midden composition and deposition. The California School of midden analysis, a group of scholars known for their dedication to using midden constituents to understand site accumulation (Waselkov 1987:141), was particularly influential. Researchers associated with the California School stressed the value of constituents like fish remains, shell, rock, ash, and residue, which had been largely undervalued prior to their work (e.g. Cook and Treganza 1947; Gifford 1916). They addressed questions surrounding past populations, including ecology and trade (Cook and Treganza 1947) while maintaining an explicit awareness of site formation processes. Scholars also debated whether weighing or counting midden constituents was best for dating shell deposits. A major concern was the impact mechanical crushing of shell would have on volumetric measurements, a testament to the consistent engagement with midden formation processes in this early research (Cook 1946). Cook and Treganza (1947) even questioned the chemical composition of shell mounds and found each one they tested to be chemically distinct. They argued for the designation of "chemical archaeology" as a new sub-field of study ${ }^{3}$ (Cook

\footnotetext{
${ }^{3}$ Although some of the earliest applications of sediment chemistry in archaeological research were conducted at shell midden (or mound) sites, chemical analysis of shell midden sediments is rare relative in these compared to other contexts, and is certainly not routine.
} 


\section{M.A. Thesis - K. Carter; McMaster University - Anthropology}

and Treganza 1947:140). This early work inspired much of the historical and continued interest in shell midden formation.

A similar approach is seen much later in Ham's (1982) work at the Crescent Beach site, which included extensive in-field investigations geared toward obtaining information about subsistence strategies and stratigraphy. Like the California School, Ham was interested in the processes of shell midden deposition. He isolated specific layers and drew inferences about seasons of occupation and the consistency of activities over time. His attention to depositional details allowed him to address questions (i.e. of seasonality and subsistence) that could not have been evaluated through artifact analysis alone.

Conover's $(1972,1978)$ work on the central coast also emphasized the value of shell midden constituents, specifically 'non-artifactual debris', for informing archaeological problems. Her work at Namu (ElSx-1), Kisameet (ElSx-3) and other smaller shell middens near Bella Bella (FbSx-6, FbTc-1 and EkSx-1) was conducted as part of a larger research program run by the University of Colorado. The Bella Bella Prehistory Project had many aims, one of which was to develop methodologies for investigating shell middens (Hester 1978:6). Conover's contribution and argument was for the integrated, systematic recovery and analysis of materials like shell, rock, ash, charcoal, bone, soil, and plant remains (Conover 1972:1, 1978:67), drawing from earlier work by Gifford (1916) (Conover 1972:29, 35). From these integrated data, Conover demonstrated long-term, continuing settlement trends over 9000 years, while highlighting variability in individual site depositional histories. Conover (1972:284) viewed non-artifactual deposits as physically patterned residues of similarly patterned behaviour.

Comparatively recent work on Pacific coast shell middens has focussed on the intensive analysis of midden components through excavation and regional testing programs. Stein (1992a) and colleagues produced abundant datasets at the British Camp site on San Juan Island. They reconstructed pre-depositional topography (Whittaker and Stein 1992) to understand the site's relationship to paleoshorelines, employing techniques like geophysical exploration, which is useful for investigating sub-surface deposits with minimal disturbance (Dalan et al. 1992). Stein et al. (1992) also employed detailed stratigraphic analyses at British Camp, using multiple analytical, stratigraphic units, including 'ethnostratigraphic units' drawn based on artifact content, and 'lithostratigraphic units' drawn based on in-field descriptions of overall content, 


\section{M.A. Thesis - K. Carter; McMaster University - Anthropology}

compared at different scales. The result was multiple working understandings of British Camp stratigraphy that could be compared regionally and within different areas of the site. Stein (1992b) also addressed two-toned stratigraphy through sediment analysis at British Camp, arguing that the difference between darker and lighter layers resulted from groundwater infiltration. Nelson (1992) further explored the archaeobotanical record from British Camp to determine whether distinctions between darker and lighter layers evident in other archaeological data were also evident in plant remains. Grain size distributions of shell (Ford 1992) and the effects of post-depositional alterations and recovery techniques on wood and charcoal assemblages (Greenlee 1992) have also been explored. Stein's work demonstrates the value of integrating multiple constituent datasets and employing innovative strategies to understand the complexities of shell midden formation.

Acheson (1995) conducted a three-year survey project on southern Haida Gwaii that investigated $245 \mathrm{~km}$ of coastline and identified 99 habitation sites, eighteen of which were selected for test excavations. The data gleaned from these test samples, in conjunction with primary archival sources, allowed Acheson to consider settlement patterning in light of the situation of sites on the landscape, including their proximity to salmon streams and relationship to sea-level, among others. Through his integrative approach, Acheson challenged the ethnographic model of Northwest Coast settlement that was widely accepted by archaeologists ${ }^{4}$ (Acheson 1995:273), arguing that small, nucleated settlements that existed prior to the fur trade eventually re-emerged as multi-lineage settlements as the result of complex processes, rather than as the result of a single event. His approach demonstrates the value of multi-site research with minimal sub-surface investigation.

Where Stein (1992a) employed the intensive analysis of materials from full-scale excavation and Acheson (1995) emphasized regional analysis using small-scale testing, Cannon (2000a, 2000b, 2013a) has intensively analyzed small amounts of material for regional investigation. I have outlined much of Cannon's work in Chapter 1 and Chapter 3, and later in this chapter. His research combines multiple archaeological indicators to discuss regional patterns in settlement histories and fisheries on the central coast. By using combined auger and core sampling, Cannon's research shows the value of integrating multiple constituents for

\footnotetext{
${ }^{4}$ See "NWC Settlement Patterns" section below for more detail.
} 


\section{M.A. Thesis - K. Carter; McMaster University - Anthropology}

highlighting variability in light of the complexities of shell midden deposition on a regional scale.

Early emphasis on site composition and the analytical potential of shell midden constituents stemmed from researchers questioning variation in site deposition processes (e.g. Cook and Treganza 1947; Gifford 1916). This work by the California School set the stage for subsequent intensive investigations aimed at understanding site formation and deposition (Ham 1982) and projects that integrated multiple constituents with the goal of informing variation and continuity in settlement patterning (Conover 1972, 1978). Recently archaeologists expanding from this trajectory have employed intensive, innovative techniques using full-scale excavation (Stein 1992a), and small-scale investigations at multiple sites to understand regional variability (Acheson 1995; Cannon 2000a, 2000b, 2013a). All share an interest in using complex shell midden deposits to their fullest archaeological potential.

My thesis fits with these types of research by exploring phosphate as a ubiquitously distributed residue of human activity. I am addressing questions at a regional scale of analysis by considering the phosphate content at multiple sites. My goal is to contribute to Cannon's ongoing research by integrating these data with existing midden constituent data, namely fish bones. My thesis in many ways shares this historical interest in shell midden constituents and supports the argument in favour of using non-artifactual materials. It also aligns with the broader literature surrounding sediment chemistry in archaeological research, to which I turn next.

\subsection{Sediment Chemistry in Archaeological Research}

\section{Applications}

Elemental analysis of archaeological soil and sediment is an established technique. Archaeologists have used it to distinguish cultural from non-cultural deposition, detect and analyze activity areas, explore spatial patterning, and address post-depositional site alterations (e.g. Entwistle et al. 2000; Griffith 1980; Middleton and Price 1996; Moss 1984; Schlezinger and Howes 2000; Stein 1987). This archaeological application rests on the premise that humans as depositional agents alter the chemistry of soil or sediment. The relative enrichment or depletion of elemental concentrations, usually compared to off-site samples, is indicative generally of human presence or absence, and combined multi-element approaches have been useful for suggesting particular activities and activity areas. 


\section{M.A. Thesis - K. Carter; McMaster University - Anthropology}

In addition to the range of techniques available for elemental analysis in archaeology, there is also a range of elements that archaeologists tend to investigate for different reasons ${ }^{5}$. Phosphorus (P) is one element that is widely investigated. It has a tendency to fix in soils and is therefore a uniquely persistent element and indicator of human activity. Variability in phosphorus concentrations can be the result of a number of different daily activities and serves as a good general indicator of human occupation (Holliday 2004:307).

\section{Phosphorus in Archaeology}

The earliest application of archaeological phosphorus analysis emerged in the late 1920s with pioneering work in Sweden by Arrhenius $(1934,1963)$ who recognized a correlation between heightened phosphate and archaeological sites, documenting the utility of the application for site prospection and delineation. In Germany, Lorch (e.g. 1940) extended this research in the late 1930s and early 1940s by distinguishing settlement types through patterns in phosphate signatures, although Bethell and Máté (1989:2) note that he was aware of the limitations of his work. These researchers adopted techniques from agricultural sciences ${ }^{6}$, originally developed to determine the amount of the nutrient available for plants to use (Holliday and Gartner 2007:313). This initial research led to the establishment of phosphorus analysis as a valuable technique in archaeology, particularly for site survey and for detecting and analyzing activity areas (e.g. Eidt 1977; Provan 1971; Holliday and Gartner 2007; Sanchez et al. 1996:152). More recently, archaeologists have also used phosphorus in conjunction with multielement analyses towards similar goals (e.g. Abrahams et al. 2010; Eberl et al. 2012; Kanthilatha et al. 2014; Lubos et al. 2016; Milek and Roberts 2013; Misarti et al. 2011; Oonk et al. 2009; Vyncke et al. 2011).

\section{Characteristics, Forms, and Fractions of Phosphorus}

Archaeological interest in phosphorus, in conjunction with research by soil chemists, has led to "a bewildering array of terms for referring to soil P" (Holliday and Gartner 2007:303). Its chemistry is also complex and not fully understood. To discuss relevant concepts and maintain clarity, I highlight the parts of the phosphorus cycle that seem to be primarily important for

\footnotetext{
${ }^{5}$ Refer to section 2.4 under heading "Humans as Depositional Agents" for more information on particular elements of interest.

${ }^{6}$ Arrhenius was a Swedish agronomist. His work is also credited as being the first systematic attempt at archaeological phosphate analysis (e.g. Bethell \& Máté [1989:1]; Sanchez et al. [1996:152]).
} 


\section{M.A. Thesis - K. Carter; McMaster University - Anthropology}

archaeologists. Such an understanding is key to interpreting P signatures in archaeological contexts (Holliday and Gartner 2007:303).

Phosphorus terminology reflects the make-up of the element (e.g. organic P, inorganic P, and total P) and its distribution in the biogeochemical environment (e.g. available P) (Bethell and Máté 1989:7; Holliday 2004:306; Holliday and Gartner 2007:303). Phosphorus cycles naturally through its availability to plants and their subsequent deposition, either through decomposition or their consumption and re-deposition by animals. Some amount of phosphorus, depending on a web of environmental factors, is also subject to leaching. Most archaeological research on phosphorus content deals with the losses and gains (depletion or enrichment) caused by human deposition that disrupts the natural cycling of soil phosphorus.

Phosphorus is either organic or inorganic. It exists in soils primarily as phosphates ${ }^{7}$, which additionally exist in different forms throughout a cycle that has multiple components and many processes (see Figure 2.1). The interplay among components of the phosphorus cycle is variable and largely dependent on local biological and chemical conditions, such as texture, moisture, and $\mathrm{pH}$ (Holliday 2004:354). All of these influence the degree to which different forms of phosphorus are mobilized or stabilized; $\mathrm{pH}$ plays a particularly large role. Phosphorus that is added to the soil/sediment bonds with iron (Fe) and aluminum (Al) ions in acidic conditions, and with calcium (Ca) ions in basic conditions (Bethell and Máté 1989:6; Dietz 1957:405).

Archaeologists talk about inorganic $\mathrm{P}$, organic $\mathrm{P}$, total $\mathrm{P}$ and available $\mathrm{P}$, which all consist of variably fixed compounds. Inorganic P can exist as an orthophosphate ion that has been occluded within particles or adsorped to them ${ }^{8}$. It can also exist as a chemical precipitate, or in soil solution (Holliday and Gartner 2007:303). Organic P exists adsorped to particles or in solution. Total $\mathrm{P}$ refers the sum of inorganic $\mathrm{P}$ and organic $\mathrm{P}$.

Available $\mathrm{P}$ describes the amount of phosphorus available for plants to use. It refers to $\mathrm{P}$ in a particular place in its cycle. Holliday and Gartner (2007:304) characterize both soluble inorganic and organic $\mathrm{P}$, and weakly adsorped inorganic $\mathrm{P}$ as part of the "available $\mathrm{P}$ pool"

\footnotetext{
${ }^{7}$ More specifically, as either a primary or secondary orthophosphate ion, but phosphate is the term used generally (Bethell and Máté 1989:5).

${ }^{8}$ Sorption is used to describe surface associations between solid soil particles and chemicals; if phosphorus is said to be occluded, it has become incorporated with/within particles physically or chemically (Holliday and Gartner 2007:303).
} 


\section{M.A. Thesis - K. Carter; McMaster University - Anthropology}

because they are mobile and susceptible to processes of dissolution, transformation, and desorption, which would render them part of the labile - or easily changed - P 'pool'. This reservoir replenishes $\mathrm{P}$ in solution as phosphorus cycles, maintaining an equilibrium. Unlike solution $\mathrm{P}$, which is readily available to plants, the labile $\mathrm{P}$ pool is potentially available ${ }^{9}$ (Bethell and Máté 1989:6).

Measurements for available $\mathrm{P}$, then, are designed to get at both the readily available solution $\mathrm{P}$ and the easily extractable labile $\mathrm{P}$ (which would give an accurate idea of the amount of the nutrient available for plants to use) (Holliday 2004:345). They may also get at the readily transformable, weakly adsorped P. As such, measurements of available P do not represent a particular form of naturally existing phosphorus (Holliday and Gartner 2007:313). Instead, determinations are only roughly indicative of the phosphorus content of a soil or sediment (Bethell and Máté 1989:5). Measurements of total P are designed to get at both mobile and relatively stable components, including $\mathrm{P}$ that is strongly adsorped or immobilized. I will discuss methods employed for measuring available $\mathrm{P}$ and total $\mathrm{P}$ in more detail in the following section.

I have included a glossary of the terms discussed above in Table 2.1. Following suggestions from Bethell and Máté (1989:5), which are further echoed by Holliday (2004:304), I use the term 'phosphorus' when referring to total P measurements because some compounds of organic P (a component of total P) are not phosphates. I use the term 'phosphate' otherwise. The fraction of $\mathrm{P}$ that archaeologists test for varies from study to study, as does the analytical method employed.

TABLE 2.1. Glossary of phosphorus related terminology.

\begin{tabular}{|c|c|}
\hline Available P & $\begin{array}{l}\text { All of the } \mathrm{P} \text { available for plants to take up; does not represent any true form of } \mathrm{P} \text { that exists in } \\
\text { nature, as it is a measurement of both solution } \mathrm{P} \text { (readily available) and labile } \mathrm{P} \text { (potentially } \\
\text { available) in a soil or sediment, which are made up of inorganic and organic } \mathrm{P} \text { compounds. }\end{array}$ \\
\hline Extractable $\mathbf{P}$ & Refers to easily extractable P. Holliday (2004:349) groups it with available P. \\
\hline Inorganic P & Inorganic compounds of phosphorus. \\
\hline Labile P & An easily changed reservoir that maintains an equilibrium with solution $\mathrm{P}$ as phosphorus cycles. \\
\hline Non-labile $\mathbf{P}$ & A stable pool that is not available for plants. \\
\hline Organic $\mathbf{P}$ & Organic compounds of phosphorus. \\
\hline Phosphate & $\begin{array}{l}\text { lonic form of phosphorus; the primary form in which P exists in soils, either as a primary or } \\
\text { secondary orthophosphate ion. }\end{array}$ \\
\hline Phosphorus & $\begin{array}{l}\text { Elemental phosphorus. Also used to talk about total P measurements, as some compounds of } \\
\text { organic } \mathrm{P} \text { are not phosphates; also used to discuss the element generally. }\end{array}$ \\
\hline Solution P & $P$ that is in solution and readily available for plants to take up. \\
\hline Total P & Organic $P+$ inorganic $P$ \\
\hline
\end{tabular}

\footnotetext{
${ }^{9}$ Because labile $\mathrm{P}$ is moveable and can become solution $\mathrm{P}$, available $\mathrm{P}$ and labile $\mathrm{P}$ are nearly synonymous.
} 


\section{M.A. Thesis - K. Carter; McMaster University - Anthropology}

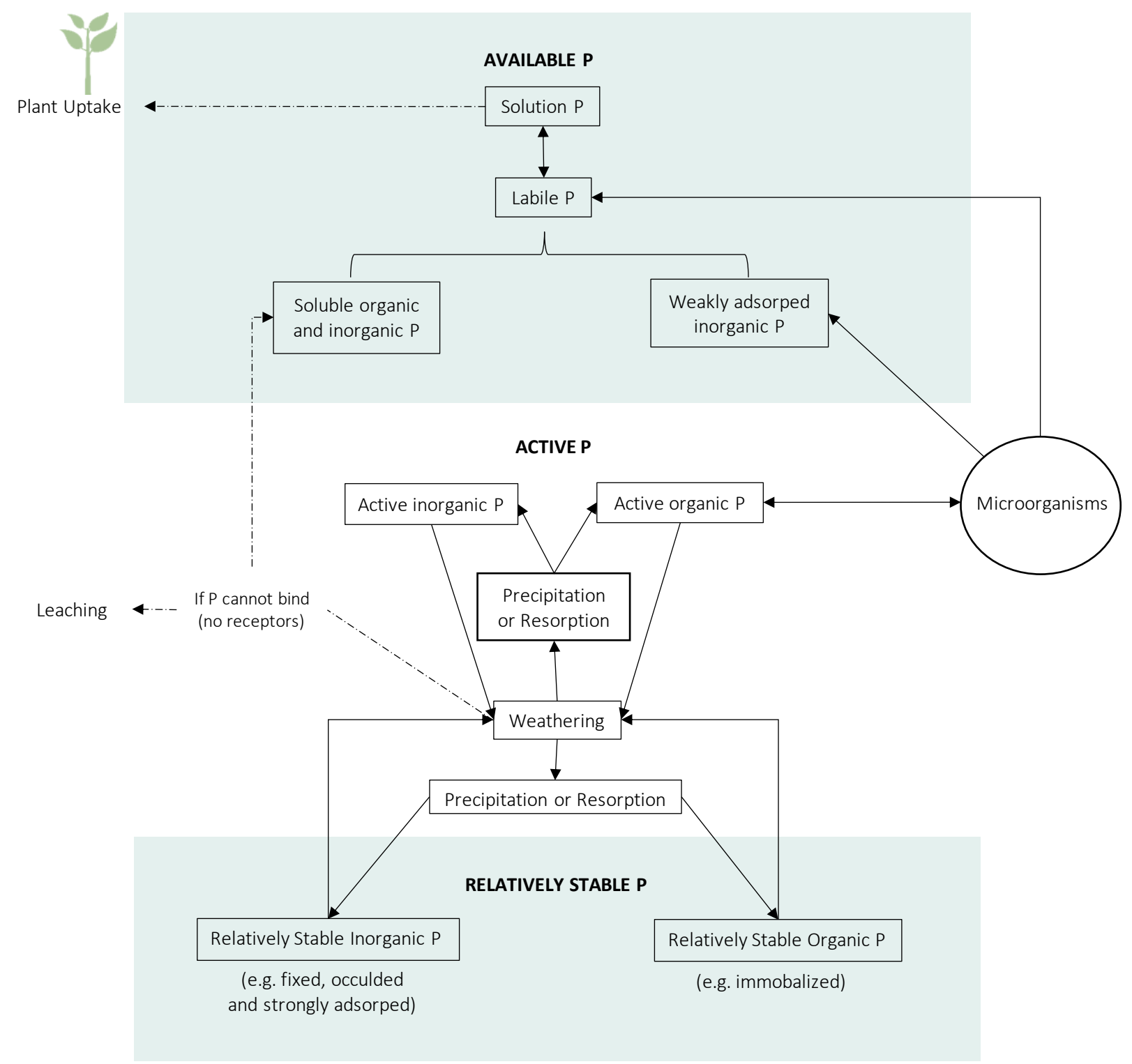

FIGURE 2.1. Pathways and interactions of the different forms and fractions of soil P. This is a simplified version of Holliday and Gartner's (2007:304) figure. All information on pathways is taken directly from that figure, and more detailed information can be found there. 


\section{M.A. Thesis - K. Carter; McMaster University - Anthropology}

\section{Measuring Phosphorus in Archaeological Sediments}

There is a variety of methods available to archaeologists for phosphorus analysis. Nearly all ${ }^{10}$ of them involve (1) extracting $\mathrm{P}$ from the soil/sediment and (2) measuring $\mathrm{P}$ in the extractant $^{11}$ (Holliday and Gartner 2007:309). Methods of analysis do this in different ways. My purpose here is to highlight the types of phosphorus archaeologists test for, why, and how, as a basis within which to situate my choices and methodological framework. I have focused this review on the geochemical and archaeological aspects that are most pertinent to my research. It is by no means exhaustive. Holliday and Gartner (2007) is a compact resource for information and references to more inclusive reviews of methods, applications, and elemental qualities of phosphorus. Holliday's (2004:346) Table A2.1 is a comprehensive overview of the available methods for extracting and measuring different fractions of phosphorus and their archaeological applications. This table provides references and lists specific chemicals used to digest and extract phosphorus in different ways, citing over two dozen methods.

I have organized my presentation generally around the analysis of available $\mathrm{P}$ and total $\mathrm{P}$ as two prominent archaeological applications. I then compare the value of assessing both forms before justifying my choices.

\section{$\underline{\text { Available P }}$}

The earliest applications of phosphorus analysis measured available or extractable P (e.g. Arrhenius 1934, 1963; Lorch 1940). Some scholars have successfully observed patterns in available P (e.g. Parnell et al. 2002) and others have questioned its utility for archaeologists (e.g. Bethell and Máté 1989).

The most central issue seems to be that available $\mathrm{P}$ does not represent a naturally existing form of phosphorus (Holliday and Gartner 2007:313). Other cited concerns include the fact that different extracts will get at different amounts of available P; "A simple water wash will get at most easily extractable $\mathrm{P}$, and progressively more vigorous techniques will yield progressively more P” (Holliday and Gartner 2007:313). Measurements also depend on a number of external factors such as water content, texture, structure and chemistry of the soil (Holliday 2004;

\footnotetext{
${ }^{10}$ Except X-ray fluorescence (XRF), which does not require extraction of phosphorus.

11 "The basic idea for extracting P from the sample is to break the bonds between P molecules and their hosts with one or more reagents" (Holliday and Gartner 2007:309).
} 


\section{M.A. Thesis - K. Carter; McMaster University - Anthropology}

Holliday and Gartner 2007). This can affect the direct comparability of different available P methods. Available P measures also cannot be used in fractionation studies ${ }^{12}$, which isolate different forms of phosphorus to address particular archaeological questions (Holliday and Gartner 2007:305).

Despite some reservations surrounding the available $\mathrm{P}$ measure, it has a continued use in archaeological research and is generally indicative of human presence (Holliday 2004:307). Terry et al. (2000) employed an easy, cost-effective, in-field method for measuring extractable P, extending from earlier, more subjective techniques, like the popular spot-test, a well-known field method for phosphate analysis used to guide excavations (e.g. Eidt 1973, 1977). The goal of Terry et al.'s study was to prospect for sites and to identify and distinguish important features. Their results showed that the Mehlich II extraction method ${ }^{13}$ worked at a sufficient resolution to detect middens.

Parnell et al. (2002) had similar success in employing this technique and are explicitly in favour of extractable elemental analysis. They compared total and extractable elemental techniques at a site in Piedras Negras, Guatemala using Mehlich II extraction with a colorimeter (Terry et al. 2000). They found that both measured forms produced important information about activity areas within the site, noting high phosphate levels within the structure they were investigating and low levels on the peripheries (Parnell et al. 2002:386). They observed a more detailed pattern between peripheries and concentrated use areas in the extractable $\mathrm{P}$ data. Based on the similarities in their results, Parnell et al. (2002:379) argue for the extractable P method due to the "resolution obtained, convenience, economy, and safety" of these methods.

\footnotetext{
${ }^{12}$ In this approach, total $\mathrm{P}$ and organic $\mathrm{P}$ measures are taken, from which inorganic $\mathrm{P}$ measures are extrapolated.

${ }^{13}$ The procedures employed by Terry et al. (2000) are very similar to the ones I used; however, the extraction methods are different. The Mehlich II 'dilute acid' procedure extracts available $\mathrm{P}$ using acetic acid $\left(\mathrm{CH}_{3} \mathrm{COOH}\right)+$ ammonium fluoride $\left(\mathrm{NH}_{4} \mathrm{~F}\right)+$ ammonium chloride $\left(\mathrm{NH}_{4} \mathrm{Cl}\right)+$ hydrochloric acid $(\mathrm{HCl})$. LaMotte's procedure, which I detail in Chapter 3, closely resembles the Mehlich I 'double acid' extraction. It uses sulfuric acid $\left(\mathrm{H}_{2} \mathrm{SO}_{4}\right)+\mathrm{HCl}$ to extract available P. In the same way that I used a testing kit made by LaMotte, Terry et al. used a kit made by Hach, which included a battery-powered colorimeter. The only advantage the LaMotte test kit seems to have over the Hach model Terry et al. used is that the LaMotte colorimeter displays a numerical value instantly. Terry et al. had to calculate their values based on percent transmittance and a standard curve. Terry et al. (2000) employed a hand-held, battery-powered colorimeter and corresponding reagents to assess available $\mathrm{P}$.
} 


\section{M.A. Thesis - K. Carter; McMaster University - Anthropology}

Although they used ICP/AES techniques, Middleton and Price (1996) preferred a mild extraction that likely produces some form of extractable or available P (Holliday 2004:353). Results from their study showed that combined multi-element analysis is useful for distinguishing archaeological features and activity areas from natural soils, from prehistoric ground surfaces, and from each other (Middleton and Price 1996:673).

Others have implemented integrated archaeological approaches using extractable elemental techniques (e.g. Birch 2016; Sullivan and Kealhofer 2004). Sullivan and Kealhofer (2004) combined phytolith and chemical analysis of archaeological site floors to distinguish patterns in horizontal activity areas. Combined with phytolith distributions at the site of Rich Neck Plantation, Williamsburg, Virginia, readily available $\mathrm{P}$ and $\mathrm{Ca}$ data were sufficient to render use-area patterns visible.

\section{$\underline{\text { Total P }}$}

As mentioned, available $\mathrm{P}$ techniques were the advent of soil phosphate analysis for archaeologists. Methods for measuring total P seem to have developed out of the need to extend the applicability of chemical analysis to instances where available P may be less appropriate (e.g. Hamond [1983], who argues that available P methods are not universally applicable to all archaeological sites) and in conjunction with technological developments - like ICP methods that allow for the rapid quantification of multiple elements, including total P (Holliday 2004:355, $357)$.

Because available P methods extract phosphates that are less fixed than those extracted by total P methods, Provan (1971:40) sought a technique that would extract a greater amount of "relatively insoluble phosphates". Holliday (2004:347) characterizes the method employed by Provan (1971) as one that may or may not extract all phosphorus in a sediment, and Provan (1971:40-41) implies that it does not. Although his results may not reflect total P, they do seem derived from methods that extract more phosphorus than available $\mathrm{P}$ methods, demonstrating a desire to measure more than available P. Provan (1971:37) found that these results supported archaeological evidence and provided supplementary information. 


\section{M.A. Thesis - K. Carter; McMaster University - Anthropology}

Provan (1971:38) also talks about a study by Johnson $(1956)^{14}$, in which a Scottish chambered cairn was analyzed for total $\mathrm{P}$ and available $\mathrm{P}$. Through these analyses, Johnson was able to discern a 'body stain' in the centre of the cairn using total P data, despite the lack of remains. In contrast, Johnson's results showed no distinction in the available P data. Provan's (1971) writings exemplify Hamond's (1983) later sentiment that available P methods are not necessarily applicable to all archaeological sites.

Until relatively recently, total $\mathrm{P}$ measures required the use of dangerous and tedious chemical procedures (Holliday 2004:354). Conway (1983), for example, employed perchloric acid digestion to assess total phosphorus at a Roman period farmstead in North Wales. He also measured extractable phosphorus to compare with total P. Conway analyzed floor deposits from three separate structures in this way. Although he consistently found that extractable $\mathrm{P}$ values showed the same pattern as total P, Conway (1983:126) was dissatisfied with the characterization of some aspects of floor deposits based on extractable P. He explicitly argued for the analysis of total $\mathrm{P}$ as a better preserved measure of original differences.

The advent of ICP techniques, and more recently X-ray fluorescence (XRF), reduced the dangers associated with total $\mathrm{P}$ analysis. Many more recent archaeological studies investigating total $\mathrm{P}$ also look at multiple additional elements potentially linked with human occupation (e.g. Entwistle et al. 2000; Misarti et al. 2011; Oonk et al. 2009)(see section on 'Humans as Depositional Agents'). Oonk et al. (2009) conducted multi-element analysis using XRF for total elemental concentrations and $\mathrm{HCl}$ extraction for easily extractable fractions. They used "total elemental concentrations (XRF data) [to evaluate] general trends in on-site element distributions, while levels of easily extractable elements (ICP-OES data) were used to highlight anthropogenic trends." (Oonk et al. 2009:1219). One aim of their study was to assess the value of each method against local lithology. They found that extractable elements were unreliable indicators of human activity in particular environments, namely calcareous clayey soils (Oonk et al. 2009:1219).

\footnotetext{
${ }^{14}$ Some authors cite this study as Johnson (1954). It is an appendix to a paper published in the Proceedings for the Society of Antiquaries of Scotland. This date for this publication is actually a range, from 1954 to 1956 , and specific dates for each paper in the volume are not specified. I followed Provan (1971) and cite Johnson (1956).
} 


\section{M.A. Thesis - K. Carter; McMaster University - Anthropology}

\section{$\underline{\text { Summary and Comparison }}$}

Available P methods in archaeology have been met with some skepticism, particularly surrounding the fact that available or extractable P measures only roughly indicate the amount of $\mathrm{P}$ in a soil or sediment and do not reflect a particular fraction of soil phosphorus found in nature. Despite this, available $\mathrm{P}$ is generally indicative of human presence. It has effectively informed archaeological problems, particularly in the horizontal analysis of use areas at single component sites, and in site prospection and delineation. Methods employed for assessing available $\mathrm{P}$ are additionally valuable in that they are often cost-effective, safe, and otherwise economically viable options for quantifying the $\mathrm{P}$ content of a soil or sediment.

Although more expensive and sometimes more dangerous, archaeologists have turned to total P measures, recognizing that not all archaeological problems can be appropriately addressed using available P. The development of more rapid, less dangerous techniques has also facilitated total P analysis, often in conjunction with multiple other elements (Holliday 2004:310).

Both available and total $\mathrm{P}$ data are appropriate for archaeology depending on the questions asked. Neither measurement seems to be inherently valuable for archaeologists. Those interested in multi-element analysis tend to investigate total P. The methods they employ (e.g. $\mathrm{XRF}$ ) will readily quantify total $\mathrm{P}$ in addition to the other elements of interest. Some analyses require a more precise dataset than do others (Johnson [1956], as cited in Provan [1971]). As such, available P methods are not necessarily appropriate for all archaeological sites. The same is true of total $\mathrm{P}$ measures, which may not be suitable for addressing all archaeological questions (e.g. Parnell et al. 2002).

Proponents of the total $\mathrm{P}$ measure are indeed asking questions that they can best answer through an understanding of that fraction; proponents of the available $\mathrm{P}$ measure are addressing questions at a different scale of analysis. The decision to test for total $\mathrm{P}$ or available $\mathrm{P}$ should be an educated choice based on resolution and cost-effectiveness. A researcher may otherwise risk applying an expensive method that generates data at such a high-resolution that patterns are unclear, or an inexpensive method that generates data that are too low-resolution to show variability. The dangers of working with certain chemicals must also be weighed. My goal was to strike a balance between obtaining meaningful results and doing so in a cost-effective, lowrisk way. 


\section{M.A. Thesis - K. Carter; McMaster University - Anthropology}

\section{A Note on Extraction \& Comparability}

As mentioned, one key component in archaeological $\mathrm{P}$ analysis is the extraction procedure, which liberates $\mathrm{P}$ from sediments so that it can be measured using either ICP techniques or through colorimetry (Holliday and Gartner 2007:309). Different reagents extract different amounts and forms of phosphorus depending on their strength and the amount of reagent used (Holliday and Gartner 2007:309). For this reason, not all methods should necessarily be expected to produce the same results. Holliday and Gartner (2007:316) completed a comparative analysis of different methods at three separate sites: Lubbock Lake in Texas, Hulburt Creek in Wisconsin, and British Camp in Washington ${ }^{15}$. They tested four methods at each site. Two methods extracted for total P and two extracted for extractable P. Some trials used a colorimeter and some used ICP. They found that different amounts of P were extracted depending on the test. At the British Camp shell midden site, some measurements were orders of magnitude higher than were others, and some strata also showed higher values depending on the extraction method (i.e. strata that were highest in P using one method were not always highest in $\mathrm{P}$ using another), though some strata exhibited the same pattern across methods (Holliday and Gartner 2007:323).

The extraction method employed in archaeological $\mathrm{P}$ analysis seems to play a particularly large role in the comparability of results. Even if two studies measure the same fraction (e.g. total P) their results are not necessarily comparable; it is important to take both extraction and measurement procedures into consideration when results are compared across studies ${ }^{16}$. Holliday and Gartner (2007:309) stress that archaeologists must "clearly spell out and reference the procedures used in order to understand what fraction is extracted and for comparison with the work of others".

\section{Phosphorus Measured in this Study}

I used an ascorbic acid reduction method that measures available phosphate. Preliminary analysis for phosphorus was also conducted on some of the same sediments tested here using $\mathrm{XRF}$, which is a non-destructive way to measure total $\mathrm{P}$ (see section 4.7).

\footnotetext{
15 This is the same study originally reported by Holliday et al. (2004).

${ }^{16}$ It is also important to take into account sources of potential error, which I discuss in Chapter 3. Chapter 3 also discusses the methods I used in detail.
} 


\section{M.A. Thesis - K. Carter; McMaster University - Anthropology}

The ease with which multiple samples could be tested in a replicable way is primarily why I chose reagent colorimetry for this research (see section 3.3 for definition and details on the tools used). Because phosphate analysis had not yet been applied at shell middens, it made more sense to test this initial application with an easy to follow, replicable, quick and inexpensive method. Also, because the colorimeter gives a single value per sample, the quantity of data produced is small. Existing patterns are likely more readily apparent than they would be with a higher resolution method, which would have produced abundant data, but also much more noise. Because I aimed to address questions at the site-level, higher-resolution measures seemed no more valuable. Terry et al. (2000:153) also make the point that, although extractable $\mathrm{P}$ values are not necessarily proportional to the total amount of $\mathrm{P}$ in a soil, "the spatial patterns of phosphate levels are important, rather than the absolute concentration".

Middleton and Price (1996:675) present an argument in favour of available P measures

for archaeologists that is worth echoing. Although they used ICP/AES techniques, Middleton and Price (1996:675) preferenced a weak extraction using dilute $\mathrm{HCl}$ over stronger treatments. They argue that "the properties of greatest interest in anthropogenic (and natural) soils are the concentrations of mobile elements.... A stronger treatment can obscure these concentrations by releasing elements more tightly bound within mineral lattices" (Middleton and Price 1996:675, emphasis added). Oonk et al. (2009:1219) and Misarti et al. (2011:1444) also reference this point. Although Holliday and Gartner (2007:313) note that available P does not necessarily measure anthropogenic inputs of $\mathrm{P}$, the point by Middleton and Price (1996) is worth restating.

In sum, the colorimetric analysis employed here is cost-effective, replicable, and quick. It measures available $\mathrm{P}$, which is broadly indicative of human activity, and may better reflect human additions of phosphate to a sediment than do total P measures (Middleton and Price 1996:675). In any case, the comparison of available phosphate is useful (Terry et al. 2000:153). Based on the cost-effectiveness of colorimetry, the resolution of this study, and its nature as a pilot project, I considered available $\mathrm{P}$ measures sufficient for this application at shell midden sites. Results demonstrate this applicability. 


\section{M.A. Thesis - K. Carter; McMaster University - Anthropology}

\subsection{Factors Influencing the Chemical Composition of Archaeological Sediments}

The goal of this section is to review our current archaeological understanding of the ways soils and sediments are chemically enriched, depleted, or transformed. I have structured this review around (1) humans and (2) other factors, including local considerations and postdepositional alterations. Where I can, I focus on shell middens. It is impossible to quantify and list all diagenetic and local (including human) effects. I present some potential contributions to particular chemical concentrations. I separate 'Humans as Depositional Agents' from 'Other Factors' merely for ease of discussion. The idea that humans are depositional agents is a premise behind the elemental analysis of archaeological sediments; post-depositional alterations are underlying processes, regardless of elemental inputs. I intend for this discussion to highlight some of the factors that may influence shell midden chemistry.

\section{Humans as Depositional Agents}

The basic premise underlying soil/sediment chemistry for archaeologists is that humans deposit things that influence chemical concentrations. Human activity is a local chemical input. Archaeologists have repeatedly observed patterns in the relative abundance of certain elements in particular activity areas compared to off-site samples. These areas (and by extension, certain activities) show tendencies towards distinct chemical signatures. Hearth features, for example, tend to be higher in potassium and magnesium than surrounding sediments (Entwistle et al. 2000).

Archaeologists have established relationships between specific inputs (i.e. activities) and specific elements by association. It is possible to derive expectations regarding which elements are likely to be enriched or depleted in particular scenarios through a review of the literature, even though the component contributions are impossible to segregate and quantify (see Table 2.2). Table 2.2 further highlights multiple activities and contexts to which phosphorus relates. This table shows that phosphorus is linked to many different kinds of activities, observed both archaeologically and ethnoarchaeologically. 


\section{M.A. Thesis - K. Carter; McMaster University - Anthropology}

TABLE 2.2. Elements and their tendency for enrichment in certain contexts. The ' $a$ ' table is adapted from Misarti et al. (2011:1442). They referenced multiple sources to construct this table. I refer the reader to their table for more information. The ' $b$ ' table is based on select ethnoarchaeological studies to highlight some more specific potential differences among elements. $\mathrm{P}$ is bolded throughout to highlight the variety of potential activities and contexts to which it relates.

a)

\begin{tabular}{|cl|}
\hline Elements & \multicolumn{1}{c|}{ Associated activity, context or material } \\
\hline $\mathbf{P}$ and $\mathrm{N}$ & Bone, organic waste, manure \\
$\mathrm{N}$ and $\mathrm{Ca}$ & Bone \\
$\mathrm{Mg}$ & Fish and bird bone, wood ash, heat treatment of rocks \\
$\mathrm{K}$ & Waste, wood ash \\
$\mathrm{Ca}$ and $\mathrm{Sr}$ & Prehistoric house floors, corn processing, food preparation \\
$\mathbf{P}, \mathrm{K}, \mathrm{Al}, \mathrm{Mg}$, and $\mathrm{Ti}$ & Hearths \\
$\mathrm{Ca}$ and $\mathbf{P}$ & Marine shell \\
$\mathbf{P}, \mathrm{Ca}, \mathrm{K}, \mathrm{Mg}$ & Fish processing areas, Kiln areas \\
$\mathrm{Fe}, \mathrm{Al}$, and $\mathrm{K}$ & Burial soils \\
$\mathrm{Mn}, \mathbf{P}, \mathrm{Mg}, \mathrm{Al}, \mathrm{Fe}$ and $\mathrm{K}$ & Fish processing areas \\
$\mathrm{Fe}, \mathrm{Ti}$, and $\mathrm{Al}$ & Natural soils, lithic production areas \\
$\mathrm{Na}, \mathrm{K}, \mathbf{P}, \mathrm{Ca}$ and $\mathrm{Mg}$ & Kitchen areas \\
$\mathrm{Ba}, \mathrm{Fe}$ and $\mathrm{Na}$ & Ovens \\
\hline
\end{tabular}

\section{b)}

\begin{tabular}{|c|c|}
\hline \multicolumn{2}{|r|}{ SELECT ETHNOARCHAEOLOGICAL STUDIES } \\
\hline \multicolumn{2}{|l|}{ Middleton and Price (1996) } \\
\hline $\mathrm{K}, \mathrm{Mg}, \mathbf{P}$ & Hearths (presumably also ash dumps) \\
\hline $\mathrm{Ca}, \mathrm{Na}, \mathrm{Sr}$ & Interior areas \\
\hline $\mathrm{Ca}, \mathrm{Sr}$ & food preparation (in this case masa), enclosed spaces \\
\hline $\mathrm{Na}$ & biological bi-products \\
\hline $\mathbf{P}$ & excrement, plant tissue, bone \\
\hline $\mathbf{P}$ and $\mathrm{K}$ & good indicators of burning \\
\hline \multicolumn{2}{|l|}{ Fernandez et al. (2002) } \\
\hline $\mathbf{P}, \mathrm{K}, \mathrm{Mg}$ & kitchen areas \\
\hline $\mathbf{P}$ & food preparation and consumption, waste disposal \\
\hline $\mathrm{K}, \mathrm{Mg}$ & hearths \\
\hline $\mathrm{Mg}$ & kitchen and refuse areas \\
\hline \multicolumn{2}{|l|}{ Frink, Knudson et al. ${ }^{*}$} \\
\hline $\mathrm{Ba}, \mathrm{Ca}, \mathrm{Mg}, \mathrm{K}, \mathbf{P}, \mathrm{Na}, \mathrm{Sr}$ & fish processing (samples from beneath drying racks) \\
\hline $\mathrm{Na}, \mathrm{Mg}, \mathbf{P}, \mathrm{Mn}$ & fish processing area features \\
\hline
\end{tabular}




\section{M.A. Thesis - K. Carter; McMaster University - Anthropology}

\section{Other Factors}

The purpose of this section is to highlight some of the other local factors and depositional alterations that might influence the chemistry of sediment, beginning with local soil and bedrock conditions. There are many post-depositional processes acting on a site and I will not attempt cover them all here. I present on three specific processes: (1) compaction, (2) groundwater infiltration and (3) leaching because they have been discussed in direct relation to shell middens and seem to affect the distribution of chemical concentrations most prominently. My assumption is that these effects are acting equally on most parts of the site and in cases where effects are unequal, they should also be recognizable in the sediment chemistry.

\section{$\underline{\text { Local Soil Formation and Bedrock Geology }}$}

Archaeologists typically use off-site samples as a baseline comparison to local soil conditions. Understanding the bedrock geology and local soil environment is usually key in determining the presence/absence of chemical signatures related to human activity. Shell middens are unique in that they consist primarily of cultural deposits. There is no expected relationship between these primarily shell deposits and the underlying bedrock (with the exception of some local additions, like fire cracked rock, or sediments incidentally brought onto and incorporated into the midden). People lived on existing shell midden deposits. They concentrated and redistributed phosphate within the midden itself, not an underlying ground. In this way, a shell midden is its own local geology.

\section{Groundwater Infiltration}

Groundwater infiltration is a post-depositional alteration known to affect the composition of shell midden sediments ${ }^{17}$ (Stein 2008). Fluctuations in groundwater also degrade organics and obscure stratigraphy, which has implications for preservation at archaeological sites (Holliday 2004:269). Groundwater can also influence soil/sediment chemistry by bringing elements onto a site and adding to the signature (Bailey 2006).

Groundwater infiltration has received special attention for its role in generating particular stratigraphy at shell midden sites, whereby lighter sediments overlie distinctly dark layers. This pattern has been observed at many NWC sites and around the world (Stein 2008). Groundwater

\footnotetext{
${ }^{17}$ In cases where infiltration actually does occur.
} 


\section{M.A. Thesis - K. Carter; McMaster University - Anthropology}

can infiltrate sites that were already located in low-lying coastal areas or those later affected by sea-level change (Stein 2008). Stein (2008) found that weathering of carbonate materials, facilitated by groundwater infiltration, was responsible for the light/dark stratigraphic boundary at the British Camp shell midden. Carbonate, as a mobile chemical compound, was removed from the lower layers. Stein's (2008) findings indicate that mobile elements may be removed from the base of shell middens that exhibit the light/dark stratigraphic boundary seen at British Camp.

The basal deposits at two sites in this study, ElSx-1 and ElSx-16, have been subjected to the effects of groundwater. Although I used site-level averages in this study, each average was generated from a number of samples from a single auger location. Because of this, I was able to assess potential differences in available phosphate within a vertical profile from both sites. XRF analysis was also conducted on the same sediments from these sites. This is a measure of the total phosphorus in a sample. The phosphate (colorimetry) profile for ElSx-16 shows that phosphate decreases in the basal deposits; the phosphorus (XRF) profile for ElSx-16 does not show the same pattern, although the overall trend is that phosphate decreases gradually toward the base. This could suggest that groundwater infiltration is influencing the colorimetry results at this site, but not the XRF results. This is likely because the XRF measurement includes relatively stable fractions (see Figure 2.1), where colorimetry does not. The phosphate (colorimetry) and phosphorus (XRF) profiles at ElSx-1 also seem to suggest that total phosphorus is better preserved than available phosphate, although the differences here are even less dramatic that at ElSx-16.

The decline in the phosphate profiles at both ElSx-16 and ElSx-1 is subtle and it is difficult to know if it has resulted from the effects of groundwater. There is no clear break as would be expected if groundwater played a major role. In any case, it seems that only the bottom three samples at both sites may have been affected, and they still show the presence of phosphate. Further, although only a potential effect at two shell middens tested here, any impacts of groundwater infiltration are averaged into single, site-level values in this study. For the purposes of this thesis, potential distinctions in the sediment chemistry due to groundwater infiltration do not significantly affect overall values and therefore the comparability of site-level measures among sites is not impacted. Based on the subtle differences between profiles at these 


\section{M.A. Thesis - K. Carter; McMaster University - Anthropology}

two sites, implications for these effects may arise in a study of vertical elemental distribution in shell midden sites using colorimetry. XRF values do not seem to be affected.

\section{Leaching}

Groundwater infiltration can evidently cause leaching of certain elements from shell midden sediments. Leaching in theory could also occur throughout the depth of the midden over time with subsequent rains. This could cause redistribution of certain mobile elements. In places with high rainfall, mobile elements may leach. The results in this thesis are interpreted on the basis that all sites in this study are located in the same biogeoclimatic zone and experience similar annual rainfall, and the focus of analysis and interpretation is on site-level averages.

Phosphorus is relatively immobile. Leaching likely has a greater impact on elements other than phosphorus and would be important to consider in multi-element analyses. That said, phosphate does have the potential to leach in some conditions. $\mathrm{P}$ binds in acidic and basic conditions; it is most susceptible to mobilization in environments with a neutral $\mathrm{pH}$ (Holliday and Gartner 2007:306). Shell midden sediments tend to be neutral to slightly basic and the sediments tested here are largely neutral (Bathurst 2005b:330-337). However, the mobilization of immobile $\mathrm{P}$ fractions does not necessarily mean that leaching has occurred on a large scale. It could mean that in neutral $\mathrm{pH}$ conditions, less $\mathrm{P}$ remains fixed and more $\mathrm{P}$ moves into the available P pool (see Figure 2.1). Also, Holliday and Gartner (2007:302-303) note "in many relatively undisturbed settings, people can add so much $\mathrm{P}$ to occupation surfaces that [soil $\mathrm{P}$ transformations and leaching] may not compromise P analysis". Results from this thesis show good preservation of available $\mathrm{P}$ in shell midden sediment. It is unlikely that leaching plays a significant role in the overall variability seen in phosphate results, but might contribute to the distribution of other elements.

\subsection{Sediment Chemistry on the Pacific Coast}

Here I review studies to date that have conducted sediment chemistry on the Pacific Coast of North America. They range in application from the analysis of midden content (Cook and Treganza 1947; Holliday et al. 2004) to site detection (Moss 1984), to ethnoarchaeological studies (Frink and Knudson 2010; Knudson and Frink 2010b) and studies of site spatial organization (Misarti et al. 2011). 


\section{M.A. Thesis - K. Carter; McMaster University - Anthropology}

Holliday et al. (2004) analyzed British Camp sediments to compare different methods for testing phosphorus ${ }^{18}$. Their work shows that the extraction method employed greatly contributes to the comparability of results. More recently, Trant et al. (2016) investigated calcium and phosphorus concentrations at and around shell midden sites on the central coast of British Columbia to assess their impact on forest productivity. As far as I am aware, these two studies and the early work by Cook and Treganza (1947) represent the only applications of chemical analysis at Pacific coast shell midden sites.

Moss (1984) conducted phosphate analysis at multiple sites on Admiralty Island in Alaska. Her goal was to identify ethnographically recorded sites through phosphate in the absence of other archaeological evidence. By comparing the phosphate chemistry of known sites and non-sites with the chemistry of suspected sites, Moss observed differences. She notes that although intra-site comparisons were limited, clear patterns emerged when similar types of sites were considered together; "distinct types of sites show similar trends" (Moss 1984:97). Moss concluded that phosphate analysis provided evidence that was useful for better understanding otherwise ambiguous sites. Her work shows the value of comparing phosphate values among different types of sites within a regional context.

Frink and Knudson have explored the value of chemical analysis for assessing patterns associated with contemporary Native fisheries on the Alaskan coast (Frink and Knudson 2010; Knudson and Frink 2010b; Knudson et al. 2004). They analyzed soil samples from salmon processing and herring processing camps for multiple elements. Their initial research compared two salmon fishing camps, which varied in length of occupation, but were used by the same extended family over decades. Knudson et al. (2004:453) found that the "length of camp occupation is associated with the strength of and variability in anthropogenic soil signatures," although they suggest more work needs to be done to elaborate on this relationship. In subsequent work, Frink and Knudson (2010) compared salmon and herring fishing camps and found that chemical analysis cannot distinguish fish species. They also conducted spatial analysis of use areas through sediment chemistry to distinguish which elements are elevated in fish processing features (Knudson and Frink 2010b).

\footnotetext{
${ }^{18}$ Holliday and Gartner (2007) discuss this same study. See above for more detailed information on their findings.
} 


\section{M.A. Thesis - K. Carter; McMaster University - Anthropology}

Misarti et al. (2011) take a unique approach to sediment chemistry analysis in the eastern Aleutian Islands, Alaska. Their goal was to investigate the value of distinguishing areas within sites in lieu of excavation. They collected and analysed multiple auger samples, using weak-acid extraction for a number of elements. They drew from correlations between activity areas and chemical signatures documented in the archaeological literature and sampled known surface features (like berms and house pits) to reconstruct activity areas. They also showed that the intensity of site occupation influences soil chemistry.

These studies represent a mix of purposes and contexts. They range in application from method evaluation and site prospection, to the identification of activity area and intensity of site occupation. Their applications also range from non-shell midden to shell midden deposits. For this thesis, I focus on known shell-midden and shell-bearing sites in order to assess the relative intensity of site occupation at particular locations.

\subsection{Settlement Archaeology}

My research investigates phosphate as an indicator of occupational intensity at shell midden sites and contributes to an understanding of regional settlement patterns on the central coast. In this section, I reference the types of questions archaeologists aim to answer when they investigate regional settlement patterns. This includes a brief historical review of settlement archaeology more broadly, but with a focus on North American approaches and interests. I turn specifically to the NWC, citing settlement patterns that archaeologists have assumed for the region, followed by a discussion of specific patterning for British Columbia's central coast based largely on Cannon and colleagues' research. In doing this, I situate my work within the framework of regional settlement pattern studies.

\section{Some Themes}

In a 1972 review, Parsons (1972) highlighted the historical trajectory of archaeological settlement pattern research. He notes that early work on settlement patterns in North America ${ }^{19}$ included dwellings, the arrangement of dwellings, and the nature of other types of buildings (Parsons [1972:128-129], citing Willey [1953]). Settlement pattern research dealt largely with architecture (Parsons 1972:128). In the mid-1950s, interests started to extend beyond

\footnotetext{
${ }^{19}$ He talks about an American and English tradition of settlement archaeology. I am restricting my brief reiteration to North America.
} 


\section{M.A. Thesis - K. Carter; McMaster University - Anthropology}

architectural features to ideas of 'community patterns', including population expansion based on subsistence. This expanded approach was more theoretical than previous work, yet Parsons (1972:129) notes that it was limited in practical application. These ideas did spark discussions surrounding the scope of settlement archaeology, stemming largely from Willey's (1956) later publication Prehistoric Settlement Patterns in the New World. Here we see the suggested introduction of regional settlement studies to the broader settlement patterns agenda (Parsons 1972:130). Initially, those interested in regional settlement patterns used direct ethnographic analogy (Parsons 1972:130). These concepts were most prolific in the American Southwest (Parsons 1972:130). By the late 1960s, three primary units of study had been established for settlement archaeology: (1) individual structures, (2) local settlements, and (3) regional settlement distributions (Parsons 1972:137; Trigger 1967:152).

For the most part, settlement archaeology has maintained, but expanded upon, these same study goals. 'Settlement' exists either as a site/house/domestic practice or as a regional pattern/action (Brück and Goodman 1999). Although under the broader umbrella of settlement archaeology, each reflects specialized interests: the former coincides with household archaeology, the latter with regional settlement pattern studies. Both theoretically engage with the idea of a 'locus' of daily activity, whether that be the household, or the broader 'site', an area of concentrated use on a landscape. Brück and Goodman note that earlier work - stemming from Willey (1953) - was biased towards the production and reproduction of site-distribution maps, which perpetuated Cartesian, capitalist ideas of space as a "series of snapshots" (Brück and Goodman 1999:7). These studies were also problematic in their use of direct ethnographic analogy. Brück and Goodman (1999) argue instead that regional settlement pattern studies are tied intrinsically to concepts of landscape.

Kowalewski (2008) teases out themes in regional settlement pattern studies, placing them into multiple groups, in a paper that summarizes works from 1996 to $2008^{20}$. In one section, Kowalewski (2008:232) notes that "one of the more productive recent research areas has been regional hunter-gatherer subsistence and settlement". He suggests three main questions or interests that archaeologists have pursued in this regard: (1) movement based on lithic raw

\footnotetext{
${ }^{20}$ Kowalewski includes an extensive works cited list and bibliography that details vast amounts of literature, for additional reference.
} 


\section{M.A. Thesis - K. Carter; McMaster University - Anthropology}

material procurement, (2) "ties between logistical strategies and social complexity", and (3) moving beyond logistical foraging concepts towards a more holistic understanding of multiple routines. He also suggests that there is promising interest in the "intersection of the mobility models employed by hunter-gatherer scholars and the models of classical regional analysis developed for permanent settlements" (Kowalewski 2008:233). In another section, Kowalewski (2008:243) talks about 'villages and communities in regions' where he notes that some regional surveys and settlement pattern analyses have explicitly aimed at contextualizing "some rather famous sites" by situating them within a broader regional context. This is the case with Cannon's (2000a, 2000b, 2013a) work in and around Namu. Kowalewski (2008:254) also notes that systematic regional survey is the primary method for gathering information that allows archaeologists to investigate patterns and generate an understanding of 'macroregional phenomena' and for providing necessary context for more local work.

Overall, settlement pattern studies - historically and more recently (Kowalewski 2008; Parsons 1972) - share an interest in both localized questions surrounding the relationship between features at specific sites, and in regional settlement patterning, within the context of specific thematic interests, such as hunter-gatherer subsistence and broader contextualization. Brück and Goodman's (1999) work exemplifies a further interest in theoretically situating settlement pattern studies necessarily alongside landscape archaeological literature, while maintaining a unique place for 'settlements' or 'sites' themselves. My thesis, then, extends and builds on these themes by contributing to ongoing research by that integrates the archaeological village at Namu into a broader regional context (Cannon 2000a, 2000b, 2002, 2013a).

\section{NWC Settlement Patterns}

In this section, I highlight settlement frameworks that have been assumed for the NWC more broadly and those that are evident archaeologically for the central coast. My discussion of NWC settlement patterns is brief, and I refer the reader to Tobiasz (2015) for a more detailed discussion and further references.

Archaeologists have typically viewed Northwest Coast settlement within an assumed 'seasonal round' (e.g. Ames 1981) - a pattern of annual, seasonal movements of people between larger and smaller locations. Large groups gathered during the ceremonial winter season and dispersed into smaller groups during the spring, summer and fall (Fladmark 1975:91). This type 


\section{M.A. Thesis - K. Carter; McMaster University - Anthropology}

of movement presumably translates archaeologically into long-term residential sites and shortterm occupation sites, respectively. Large 'winter' villages could be occupied year-round by fluctuating numbers of people, whereas short-term sites would have been occupied by small groups annually, at around the same time each year (Burchell 2013:iv). This framework is based largely on the ethnographic record.

Archaeologists have challenged the uncritical application of ethnography to past settlement patterns (e.g. Acheson 1995; Ford 1989), and research has also shown that there is much more variability among sites in terms of their subsistence and seasonal movements than is suggested by the 'typical' model (e.g. Burchell et al. 2013; Mitchell and Donald 1988). Considering locally specific differences along with shared continuities allows for a more holistic understanding of regional settlement systems.

\section{Central Coast Settlement Patterns}

Cannon's on-going work in the Namu vicinity and adjacent Rivers Inlet has both provided context for Namu, and documented variability in the focus of activities and the relative intensity of occupation at a range of sites in the region (Cannon 2013a). The goal of Cannon's initial investigation near Namu was to understand a history of local fisheries and to relate that understanding to a regional settlement pattern. He found that indicators of marine subsistence like shell and fish bone - showed considerable variation among different locations in the area (Cannon 2000a; Cannon et al. 2008). Investigations at Rivers Inlet were undertaken to compare patterns documented in the local Namu fisheries with a different region. Rivers Inlet is comparatively rich in sockeye and contains areas that were important sources for eulachon (Cannon 2013a:25) - a widely traded, oil-rich fish. Essentially, Rivers Inlet consists of shell midden sites that are different in their composition and form, yet similar in their fish bone content, which showed evidence for similarly productive salmon and herring fisheries (Cannon 2013a:25; Cannon et al. 2011). Through investigations at both of these regions, Cannon (2013a) demonstrated that fish bone density and site area, which showed variable trends among fisheries, are good indicators of occupational intensity.

A settlement pattern that has emerged for the central British Columbia coast through this research includes a regional tendency for localized fishing oriented largely towards salmon. This includes a range of occupations of different intensities, including small settlements that match 


\section{M.A. Thesis - K. Carter; McMaster University - Anthropology}

the resources of specific locations, and sustained villages, that speak to a degree of long-term continuity (Cannon 2002:324; Cannon et al. 2011:71). Individual sites show a specific history of development that resulted from contingent circumstances, some of which are readily understood based on the ubiquity of some midden contents (Cannon 2002). In this case, fish bones can speak specifically to trends in both local and regional fisheries.

The ubiquity of fish bones also means that they can speak to general trends, as Cannon (2013a) has shown. Their deposition results from fishing, but as densities standardized by litre of fine fraction matrix, they provide evidence of how intensively a place was occupied. Especially when combined with site area, they indicate a range of variably intense occupations from shortterm camps to long-term residential sites.

Phosphate can be compared against fish bone densities to speak to these trends. Comparing elemental concentrations against densities of materials known to relate to human occupation has been a useful application at some archaeological sites. For example, Parnell et al. (2001) demonstrated a positive correlation between phosphate concentration and ceramic densities (as an indicator of residential occupation) across a site in Guatemala. They defined residential areas through their comparisons, which were aimed specifically to identify middens and target excavations. Beyond their intended aims, their research shows that variability in the spatial distribution of ceramic densities corresponded with phosphate and was useful for determining areas of occupation. They note that variability in phosphate concentrations at different areas of the site, which are positively correlated with high ceramic densities, could be due to either the intensity or length of occupation (Parnell et al. 2001:863). Another example exists in the work by Schlezinger and Howes (2000), who used elemental ratios and lithic debitage densities to address intra-site variation in the intensity of use. They used debitage as an indicator of occupation, and significant correlations between densities of debitage and C:OP (carbon:organic phosphorus) ratios, showing that the relative enrichment of $\mathrm{C}$ compared to OP may be useful indicators of occupation. Their study focussed on intra-site variability in vertical profiles, but the correlations drawn between elemental indicators and lithic debitage densities still addressed variation.

Building from the work of scholars like Parnell et al. (2001) and Schlezinger and Howes (2000), this thesis contributes the perspective of soil chemical analysis to the understanding of 


\section{M.A. Thesis - K. Carter; McMaster University - Anthropology}

central coast settlement patterns by comparing phosphate against densities of fish bone as a means to indicate the intensity of residential occupation. Frink and Knudson (2010) have specifically used elemental analysis to address Arctic fisheries, and have shown correlations between elemental signatures and fish processing locations (Knudson and Frink 2010b). Their work provides additional basis for the assumption that this technique is appropriate for addressing questions in fisheries settlement research.

\subsection{Background Summary}

To summarize, my thesis builds from and adds to existing studies of:

1) Northwest Coast shell middens, particularly their composition;

2) Sediment chemistry in archaeological research, by investigating available phosphate;

3) Sediment chemistry on the Pacific Coast of North America, and British Columbia's central coast specifically, where such analyses are not routine; and

4) The study of regional settlement patterns. 


\section{Chapter 3: Methodology}

\subsection{Overview}

My report on methods here is four-fold. I start by describing how the samples I analyzed were obtained and processed prior to my work with them. Next, I outline the basic premises of colorimetry and the specifics of the colorimeter. I describe my laboratory procedures, from subsampling and sifting to elemental analysis through colorimetry, and end by discussing how I managed the challenges I encountered.

\subsection{Sample Context}

The matrix I analyzed was obtained by Cannon (1997, 1998b, 2000a, 2000b, 2013b), who employed a program of bucket-auger and core sampling at thirty sites over the course of six field seasons as part of a regional scale project investigating variability in the form and intensity of settlements in the region $^{21}$. I accessed auger samples from nineteen of these sites for my analysis $^{22}$. These represent the known range of variability evident in site size, content and distribution on the central British Columbian coast.

Cannon collected auger samples using a hand-operated bucket-auger seven centimeters in diameter. Auger samples are taken by repeatedly hand drilling a bucket-auger into the same location until the furthest depth of the archaeological deposits is reached. This produces multiple samples representing approximately 7 to $15 \mathrm{~cm}$ arbitrary levels (Cannon 2000a:729). I analyzed each of these 7 to $15 \mathrm{~cm}$ samples for phosphate and averaged them to obtain one value per site. Each sample was bagged in the field. Initial processing involved washing the matrix in the lab through $2 \mathrm{~mm}$ mesh. The washed residual sediment was collected and left to dry ${ }^{23}$ (Cannon 2000a). I analyzed sub-samples from this previously disaggregated and processed $<2 \mathrm{~mm}$ auger matrix.

The fish bone densities used in this thesis are those previously calculated by Cannon and presented in much of his published work on the central coast of British Columbia. Cannon

\footnotetext{
${ }^{21}$ See Chapters 1 and 2.

${ }^{22}$ ElSx-1 (Namu), ElSx-3 (Kisameet), ElSx-4, ElSx-5, ElSx-8,ElSx-10, ElSx-16, ElTa-3, ElTa-18, ElTb-1, ElTb-2, ElTb-10 (McNaughton), EkSt-1 (Katit), EjSw-1 (Cockmi), EjSv-5, and EjSv-10.

${ }^{23}$ It is possible for very easily extractable $\mathrm{P}$ to be removed using a simple water wash (Eddy and Dregne 1964), but since all the samples were treated the same, any effect should be equal.
} 


\section{M.A. Thesis - K. Carter; McMaster University - Anthropology}

calculated fish bone densities as number of identified specimens (NISP) per litre of fine fraction matrix $(<2 \mathrm{~mm})$. Fine fraction matrix was used to avoid the effects of full or coarsely broken shell, which contribute disproportionately to the overall volume (Cannon 2000a:731). Cannon's (2000a) publication shows that fish bone densities and auger sampling can be used as a way to assess trends in fisheries, showing further that "auger sampling is an effective alternative to fullscale excavation for monitoring trends" (Cannon 2000a:732) in this context. I refer the reader to Cannon (2000a) for more detailed information on fish bone density calculation and auger sampling, and to numerous other publications (e.g. Cannon 2002, 2013a; Cannon and Burchell 2016; Cannon and Yang 2009; Cannon et al. 2011) for a history of auger sampling applications at shell middens.

\subsection{Basic Principles of Colorimetry and the SMART3 Colorimeter}

The fact that we perceive different wavelengths of light as colour is the basis for colorimetric analyses. Reagents are used to generate a particular colour or shade upon reaction that is indicative of the amount of a specific element in a sample. Elemental concentrations can be quantified by comparing the colours of reacted samples against colour chips or charts, or by using a colorimeter. Colorimeters are designed to read light wavelengths and can be calibrated to report results back in terms that reflect that calibration (e.g. in parts per billion of an element). Some colorimeters require the user to design and input calibrations, and others come precalibrated and only require occasional standardization.

I chose a pre-calibrated colorimeter (LaMotte's SMART3) because it was user friendly, reasonably priced, and had the capacity to test for phosphate, in addition to other potential elements. All the required reagents also came pre-packaged, which was useful. Each reagent kit or system came with the chemical reagents required for use with the SMART3 colorimeter's predesigned calibration. This meant that I did not need to order individual chemicals or design a corresponding calibration. This particular colorimeter is also hand-held and portable ${ }^{24}$.

\footnotetext{
${ }^{24}$ Although not the goal of my research, this could have implications for in-field application.
} 


\subsection{Laboratory Analysis}

Laboratory analysis was undertaken in two stages: sub-sampling and elemental analysis. Each procedure is outlined below and includes measures taken to avoid cross-contamination of samples at each stage.

\section{Sub-sampling procedure}

Each $<2$ mm bulk sample represents a single, vertical sediment collection (i.e. one 7-15 $\mathrm{cm}$ interval). I first divided these samples into more manageable volumes using a sample splitter, which divides sediment poured through the splitter equally in two (see Figure 3.1). The amount of fine fraction material available for splitting varied from sample to sample. The number of times a sample was split depended on the original volume (more sediment led to more splitting). Generally, the subsampled volume is about $1 / 8$ the original volume of $<2 \mathrm{~mm}$ sediment. After splitting, I dry-screened subsamples through $0.6 \mathrm{~mm}\left(600 \mu \mathrm{m}, 0.025\right.$ ”) $\mathrm{mesh}^{25}$. This produced two fractions of sediment. The $<0.6 \mathrm{~mm}$ fraction was retained for elemental analysis. The other fraction (which contains 0.6-2.0 mm sized particles) was kept separate, catalogued and returned to storage.

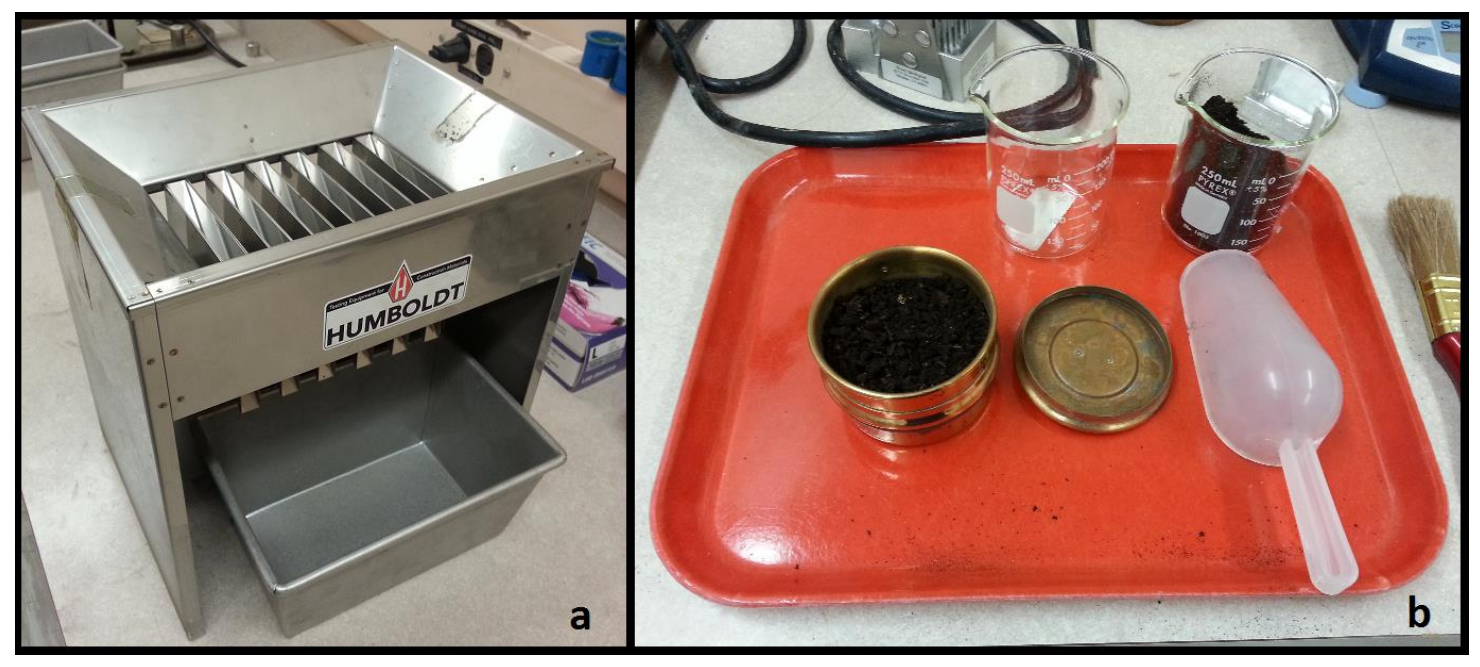

FIGURE 3.1. Sub-sampling procedures: a) Humboldt sample splitter, b) sifting through $0.6 \mathrm{~mm}$ mesh.

Sample splitting and subsequent sifting were completed in sequence for each sample (i.e. I split a sample and then immediately sifted it). This allowed for efficient processing of the samples. It also helped to maintain consistent cleaning in the lab. After each sample was

\footnotetext{
${ }^{25}$ See "Issues and Potential Sources of Error - Mesh Size" for why I chose this screen size.
} 
processed, I dusted and prepared all equipment for use with the next sample. I also processed samples in the order that they would have been collected in the field (i.e. from top to bottom) as an extra caution to avoid mixing.

\section{Colorimetric Analyses}

I used an ascorbic acid reduction method that is applied to a sediment extract. The calibration for this reagent system comes preprogrammed in the SMART3 Colorimeter. The procedures detailed below follow those provided by LaMotte in their SMART3 Soil Operator's Manual.

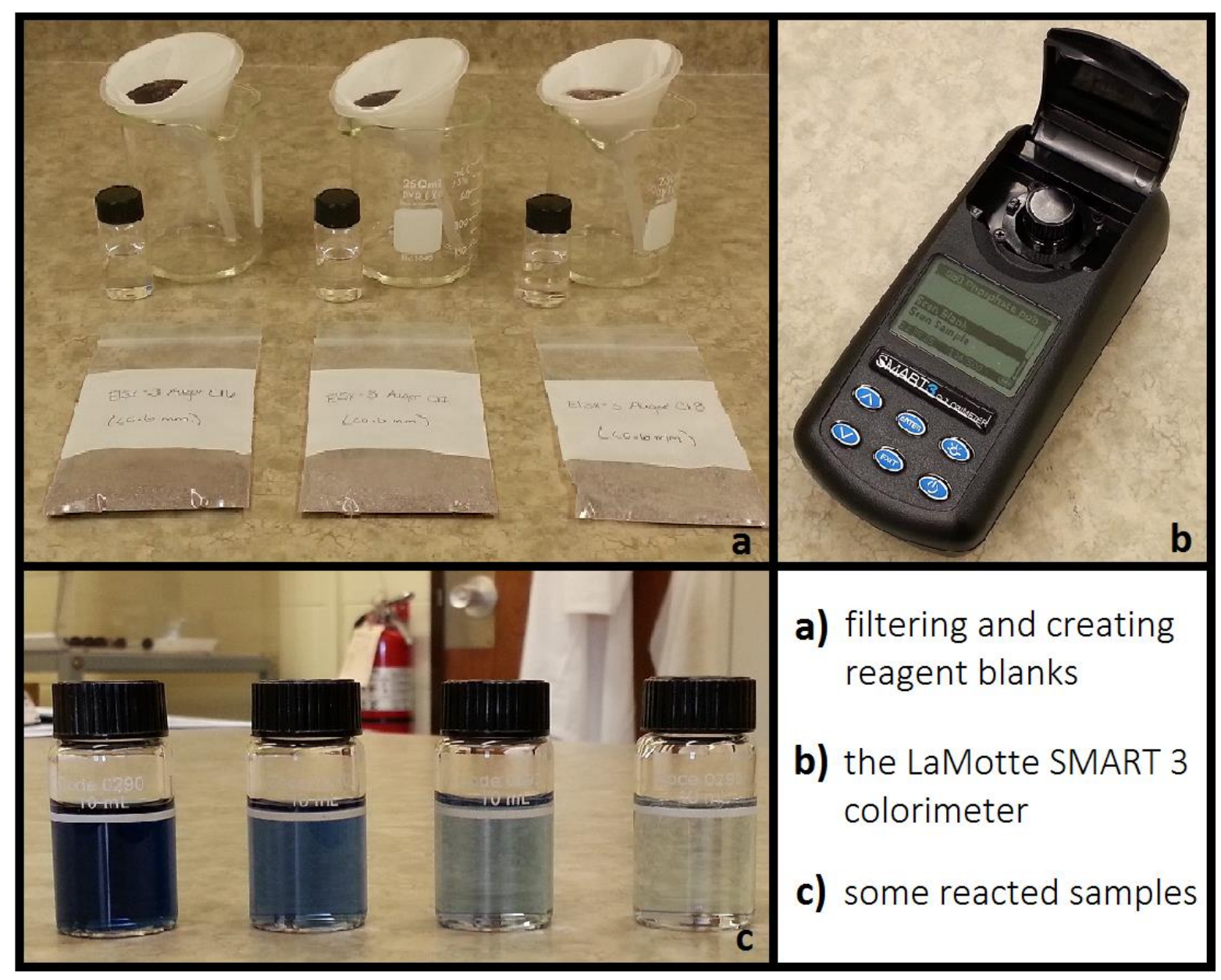

FIGURE 3.2. Some laboratory procedures.

The extraction procedure used an 'acid extracting solution' made of $<3 \%$ hydrochloric acid and $<2 \%$ sulfuric acid (with water to $100 \%$ volume). To create the extract, $1 \mathrm{~mL}$ of this extracting solution, made to a $15 \mathrm{~mL}$ volume with distilled water, is added to a vial along with 


\section{M.A. Thesis - K. Carter; McMaster University - Anthropology}

roughly $3 \mathrm{~g}$ of sediment ${ }^{26}$. The mixture is shaken for five minutes and then filtered through filter paper $^{27}$, resulting in a clear extract for analysis. One $\mathrm{mL}$ of this extract is added to a second vial, along with distilled water to $10 \mathrm{~mL}$ to produce the reagent blank, which is scanned by the colorimeter to create a baseline. After the blank is scanned, $1 \mathrm{~mL}$ of a phosphate acid reagent $(<$ $0.05 \%$ antimony potassium tartrate, $1 \%$ ammonium molybdate tetrahydrate, $5 \%$ sulfuric acid, and water to $100 \%$ ) is added to the blank and the vial is shaken. Then $0.1 \mathrm{~g}$ of a powdered phosphate reducing reagent (10\% isoascorbic acid and $90 \%$ sucrose) is added to the vial. At this stage, the vial is shaken until the reducing agent is dissolved. The vial is left to stand for five minutes to allow for full colour formation (see Figure 3.2). This reacted sample is then scanned using the colorimeter, which is calibrated to report results back in parts per billion (ppb). All glassware was washed with a clean, non-abrasive sponge and water, rinsed thoroughly again with distilled water, and left to dry between tests.

The reagent kits come with a $0.1 \mathrm{~g}$ plastic spoon for the reducing agent and a $1 \mathrm{~mL}$ plastic pipette for the phosphate acid reagent. The SMART3 colorimeter comes with multiple 10 $\mathrm{mL}$ glass vials that fit into the window of the colorimeter (see Figure 3.1). LaMotte also provides spoons, vials, and filters that can be purchased additionally through their suppliers. Their labware is numerically coded to correspond with the Soil Operator's Manual (e.g. the Manual may state "Use the $1 \mathrm{~mL}$ pipet (0354)..."). This is an efficient system for straightforward user instructions. The lab I worked in was already stocked with $1 \mathrm{~mL}$ glass pipets, $1 \mathrm{~g}$ spoons, and $125 \mathrm{~mm}$ diameter filter paper, so instead of purchasing the coded spoons, vials, and papers from LaMotte, I used what was already available. Table 3.1 lists and describes all the materials I used.

TABLE 3.1. Lab-ware used in this study.

\begin{tabular}{|c|c|c|c|}
\hline Material & Size & Brand Information & Other Info. \\
\hline Plastic spoons & $1 \mathrm{~g}$ & $\begin{array}{c}\text { Sterileware (from Bel-Art } \\
\text { Products) }\end{array}$ & Used to measure three equal parts sediment \\
\hline Glass pipettes & $1 \mathrm{~mL}$ & Kimble & Used to measure acid extracting solution and sample extract* \\
\hline Glass pipettes & $10 \mathrm{~mL}$ & Pyrex & Only used to run standards, never used with samples. \\
\hline Plastic vials & $15 \mathrm{~mL}$ & Falcon & Used to mix sediment with acid extracting solution \\
\hline Filter papers & $125 \mathrm{~mm}$ & Whatman & Used to create clear extract for analysis \\
\hline Plastic pipettes & $1 \mathrm{~mL}$ & LaMotte & Used to measure phosphate reducing reagent \\
\hline Glass vials & $10 \mathrm{~mL}$ & LaMotte & Fits into colorimeter; vial houses reagent blank and subsequent reaction \\
\hline
\end{tabular}

\footnotetext{
${ }^{26} \mathrm{I}$ say "roughly" because a $1 \mathrm{~g}$ scoop is used to add three equal parts of the material to the extract, the sediments are not weighed to 3 grams.

${ }^{27}$ I used $125 \mathrm{~mm}$ filter paper.
} 


\section{M.A. Thesis - K. Carter; McMaster University - Anthropology}

\section{Replicability}

To measure whether the colorimeter was producing consistent results, I ran a known phosphate standard every ten samples ${ }^{28}$. Over the course of my analysis, the colorimeter measured the standard consistently, so the specific machine I was using did not deviate. LaMotte's Operator's Manual (2016) includes a procedure to standardize the calibration if the user finds that the machine is not performing properly, but this was not applied because of the consistency of the standard test results. Where possible ${ }^{29}$, I also did standard additions as another check. Standard additions are a common method of testing a colorimeter's accuracy. To do this, a sample is run to determine its concentration. Then it is spiked with a known standard and run again. If the colorimeter is reporting properly, the result should be the sum of the original sample concentration and the known standard concentration. For example, if I run a sample that reads at $1200 \mathrm{ppb}$ and subsequently spike it with a known concentration of $1500 \mathrm{ppb}$, then the colorimeter should report a value of $2700 \mathrm{ppb}$.

\subsection{Issues and Potential Sources of Error}

Although this method is straightforward, I did encounter some potential issues. These and my responses did not affect the comparability of my sample results since they were consistent throughout the analyses. It could affect the comparability with other studies if these issues are managed differently ${ }^{30}$. I am presenting these as a pre-emptive caution to record, report, and consider these particulars in any effort to replicate this study.

\section{Mesh Size}

I chose to sift these sediments through $0.6 \mathrm{~mm}$ mesh because the tendency in the archaeological literature is to sort out larger components. Particles less than $2 \mathrm{~mm}$ in size are often deemed sufficient (e.g. Frink and Knudson 2010; Middleton 2004; Misarti et al. 2011; Oonk et al. 2009), but sometimes the $<1 \mathrm{~mm}$ fraction is preferred (e.g. Cook et al. 2005). The LaMotte guidelines do not suggest sifting to any particular grain size. In theory, the fraction size

\footnotetext{
${ }^{28}$ LaMotte (2016) recommends "the user periodically verify the performance of the meter by running standards with a predetermined concentration."

${ }^{29}$ I tried to do this once per site tested, but this was not always possible. The phosphate values for some samples were too high to spike; that is, the addition of the standard would have pushed the sample concentration outside of the colorimeter's range, so it would have been impossible to quantify the result.

30 There are also contextual reasons why samples or studies would not be comparable. Comparing shell midden sites to non-shell midden sites, for example, would be inappropriate.
} 


\section{M.A. Thesis - K. Carter; McMaster University - Anthropology}

should not affect the amount of available $\mathrm{P}$ in a sample ${ }^{31}$, but different extractions do produce varying amounts of $\mathrm{P}$ and some get at more mineral-bound $\mathrm{P}$ than do others ${ }^{32}$ (Holliday 2004:307). I knew that the hydrochloric acid/sulfuric acid extraction I used removed available or extractable $\mathrm{P}$, and although it is likely not strong enough to remove any mineral-bound $\mathrm{P}$, I was not certain. I also knew I had the potential opportunity for multi-element analysis using XRF, where it is often important to use a small size fraction (e.g. Kanthilatha et al. 2014; Oonk and Spijker 2015). By sifting each sample through $0.6 \mathrm{~mm}$ mesh, I removed comparatively large bits of shell and rock, ensuring a relatively uniform grain $\operatorname{size}^{33}$ and reducing future processing time.

\section{Over Range Correction}

The SMART 3 Colorimeter comes pre-calibrated to measure particular elements within a certain range. For phosphate, that range is 0-3000 ppb. If results report over that range, the colorimeter should display an 'over range' message. Although I did have samples that read over range, I actually never saw this message ${ }^{34}$. Instead, the colorimeter displayed a value that was out of range. When I inquired about this, LaMotte said that the colorimeter will still report an estimated value and that this value should be viewed with caution. The suggested procedure for dealing with an over-range value is to dilute the sample and test it again (LaMotte 2016:49). I conducted this procedure using one-half dilution, one-fourth dilution, and one-tenth dilution factors. I experimented with this procedure on multiple samples and although theoretically the results produced are closer to the absolute value of available phosphate in the sample, I could not repeat $^{35}$ the procedure with precision. The process of dilution may have hindered my ability to repeat the test, especially using different dilutions, as small errors in measurement may have been exaggerated.

I chose to use the estimated values produced by the colorimeter in cases where the results were over range. This way, even though the absolute values may not be as precise as those that

\footnotetext{
${ }^{31}$ It could affect the total $\mathrm{P}$, given that a larger proportion of mineral phosphate housed in these larger grains would add to the overall quantity of total $\mathrm{P}$.

${ }^{32}$ See "Numerical Values" section below for a more thorough discussion of this.

${ }^{33}$ The samples were already $<2 \mathrm{~mm}$ in size. I found that visibly the $0.6 \mathrm{~mm}$ mesh reduced the volatility in overall grain size. I did not actually measure the grain size proportions in the $<2 \mathrm{~mm}$ and $<0.6 \mathrm{~mm}$ sediments to compare, but the $0.6 \mathrm{~mm}$ fraction looks comparatively more uniform than the $2.0 \mathrm{~mm}$ fraction, for whatever it is worth.

34 This was just a fault with the colorimeter I was working with. LaMotte said that it was not an issue and that I should simply watch for results reading over $3000 \mathrm{ppb}$, instead of relying on the error message.

35 That is, a single person (myself) using the same sample, with the same instrument in the same lab period.
} 


\section{M.A. Thesis - K. Carter; McMaster University - Anthropology}

fell within range, they were all treated the same; the calibration is estimating the over-range values the same way for all samples. Because I aimed to compare these values with respect to their relative ranks, rather than their exact numeric measurement, the precise values were less important.

Relative comparison of elemental values (i.e. higher or lower than one another or off-site samples) has always been the premise guiding archaeological sediment chemistry, even when higher-resolution methods are employed. A scale of relative comparison within the numerical range of values I observed can be drawn clearly, and meaningful patterns are evident in the results. The strength of this method rests in the relative comparability of the results, not their precise values $^{36}$. Archaeologists are aware of individual contributions of phosphorus to a soil or sediment, but we cannot tease out and quantify them ${ }^{37}$. For this reason, the over-range correction does not impede the relative comparison of results in this study; out of range are still higher than those that are within range.

\section{Numerical Values}

There is also uncertainly around exactly what these phosphate values mean. Holliday et al. (2004) conducted a study in which they compared four different methods for extracting and measuring phosphorus at four different sites. They used a perchloric acid digestion for total P, a sulfuric acid digestion meant for total $\mathrm{P}^{38}$, a hydrochloric acid digestion for extractable $\mathrm{P}$, and a citric acid extraction for available P (Holliday et al. 2004:371). One of the sites they tested with these methods was the shell midden site at British Camp (Stein 1992a). Results from their study showed variation in phosphate values depending on the method used (Holliday et al. 2004:371). They suggest that comparability between different measurements of phosphorus analysis should not be expected. This implies a level of uncertainty surrounding phosphorus values for archaeologists that is unresolved. The literature suggests certain activities that may deposit relatively more phosphorus than other activities (e.g. Cook and Heizer 1965; Holliday and

\footnotetext{
${ }^{36}$ See Chapter 4, p. 49 for a more detailed discussion of this.

${ }^{37}$ That is not to say this should be the goal. The patterns archaeologists see are insightful, we just cannot say where the phosphorus that makes up our numbers comes from, or why that particular amount of phosphorus is being measured.

${ }^{38}$ Although it may not extract all the $\mathrm{P}$ in a sample.
} 
Gartner 2007; Knudson and Frink 2010a; Misarti et al. 2011; Moss 1984) but it remains impossible to know and quantify exactly what those contributions are.

It is also difficult to conceptualize the precision of the colorimeter. Although the SMART3 reports results in parts per billion to the nearest one, the results I generated were not always repeated with that precision ${ }^{39}$. All graphs and charts in this thesis use values rounded to the nearest hundred. I have included both non-rounded and rounded values for all samples in Appendix D: Raw Data.

${ }^{39}$ Occasionally I would scan the same reacted sample twice, just to see what number the colorimeter reported. Sometimes the number would be the same, sometimes it would be off on the tens or ones place by a few parts per billion. 


\section{M.A. Thesis - K. Carter; McMaster University - Anthropology}

\section{Chapter 4: Results}

\subsection{Overview}

As outlined in previous chapters, fish bone densities (FBD) and site area indicate clear patterns of variability in the nature of occupation at different locations. Identified villages presumed to have been occupied for longer periods by more people tend to be larger and have a greater density of fish remains, while small sites presumed to have been occupied for less time or less frequently, likely by smaller groups of people, are smaller and exhibit a much lower fish bone density (Cannon 2013a:25). I expected phosphate values would closely resemble FBD as an independent indicator of occupational intensity. Like FBD, phosphate should be higher at more intensively occupied sites and lower at less intensively occupied sites; that is, it should distinguish a range of residential occupations, from longer-term villages to short-term camps. Overall, results fit expectations. Relative phosphate values reliably indicate varying degrees of occupational intensity among sites. Phosphate can also distinguish sites associated with fish traps.

All the sites tested here are either 'true' shell middens or shell-bearing sites (Lyman 1991:39-41). They all contain different varieties and concentrations of shell, speaking to the variability in the character and formation of these places (Cannon et al. 2008). Descriptions based on differences in shell content (i.e. whether a site can be readily classified as a 'shell midden', 'shell-bearing', or 'non-shell' site) are useful in that they recognize different kinds of deposition at different places. Because each of these sites is the product of different activities and formation histories, there is no expectation that their content and deposition patterns should be the same. Rather, the expectation is precisely the opposite. There should be variability in the content - phosphate included - among all of these sites. I have referred to shell midden and shell-bearing sites collectively for ease of discussion; they are different but comparable.

I begin this chapter by presenting the results of phosphate analysis. I point out trends evident in the data, which are, as expected, variable. I then demonstrate through statistical comparison the relationship between phosphate, fish bone densities and site area. This provides the initial basis for exploring further meaningful comparisons among sites. After assessing how well phosphate fits with previously observed patterns based on FBD and site area, I discuss both the representativeness and generalizability of these results by addressing the following questions: 
(1) is phosphate generally representative of site conditions even if it is only measured from a single location, and (2) can this application be extended beyond the sites tested here?

\subsection{Phosphate Results}

Table 4.1 shows all the phosphate results generated for this thesis as site-level averages (each measured value that I used to generate these averages is listed in Appendix D). I have ordered them from highest to lowest phosphate value. There is a wide range of values from highest to lowest, though most cluster in the middle. Based on previous investigations of the content and character of these sites, phosphate values span the range of expected variability. It is clear from the distribution that phosphate represents a trend in the overall make-up or character of these sites. The expectation was that varying degrees of occupational intensity account for the particular distribution seen here.

TABLE 4.1. List of all sites tested in this study from highest to lowest phosphate content.

\begin{tabular}{|c|c|c|c|c|c|c|}
\hline Site & Site Name/Location & Phosphate (ppb) & Max. $^{1}$ & Min. $^{1}$ & $\mathrm{SD}^{2}$ & $\mathrm{CV}^{2}$ \\
\hline EkSt-1 & Katit & 11400 & 12500 & 9500 & 1323 & 0.12 \\
\hline EITa-21 & Kildidt Lagoon & 9700 & 12000 & 5000 & 2885 & 0.3 \\
\hline ElTb-2 & Spitfire Channel & 7300 & 13200 & 2900 & 4166 & 0.57 \\
\hline EISx-1* & Namu & 6400 & 12300 & 2700 & 2664 & 0.41 \\
\hline EITa-3 & Watt Bay & 6200 & 12200 & 2200 & 3536 & 0.57 \\
\hline EISx-1 & Namu & 5400 & 8900 & 4400 & 1298 & 0.24 \\
\hline ElTb-10 & McNaughton & 5200 & 11600 & 2100 & 2130 & 0.41 \\
\hline EITa-18 & Kildidt Inlet & 5000 & 6100 & 3700 & 899 & 0.18 \\
\hline EISx-3 & Kisameet & 3900 & 7400 & 1400 & 1359 & 0.35 \\
\hline EISx-18 & Fougner Bay & 3800 & 5400 & 2100 & 1103 & 0.29 \\
\hline ElTb-1 & Nulu & 3800 & 5700 & 1200 & 1120 & 0.29 \\
\hline EISx-10 & Fougner Bay & 3800 & 6200 & 2500 & 900 & 0.24 \\
\hline EjSw-1 & Cockmi & 3700 & 6000 & 1400 & 1560 & 0.42 \\
\hline EjSv-10 & Taylor Bay & 3400 & 5200 & 2000 & 1274 & 0.37 \\
\hline EjSv-9 & Taylor Bay & 3200 & 4800 & 900 & 1324 & 0.41 \\
\hline EISx-5 & Windsor Cove & 2800 & 4300 & 1000 & 1308 & 0.46 \\
\hline EISx-8 & Fougner Bay & 1500 & 4500 & 400 & 1407 & 0.96 \\
\hline EjSv-5 & Edna Matthewes Island & 1400 & 1100 & 1800 & 238 & 0.17 \\
\hline EISx-16 & Fougner Bay & 1400 & 3600 & 300 & 1103 & 0.8 \\
\hline EIS $x-4$ & Windsor Cove & 600 & 1600 & 100 & 518 & 0.84 \\
\hline
\end{tabular}




\section{M.A. Thesis - K. Carter; McMaster University - Anthropology}

What is both unclear and impossible to know is the reasons for the precise numerical values that constitute these data. Because there is an expectation for variability in the content of shell middens based on differences in their depositional histories and activities that took place over sometimes thousands of years, attempting to understand these results on an absolute basis i.e. accounting for their precise values - is unprecedented. Individual contributions to phosphate values cannot be isolated or quantified. Although I have presented averages and standard deviations, phosphate values actually become meaningful when they are compared based on their relative positions on a scale.

Drennan (2009) discusses this idea, noting that sometimes variables that seem to be true measurements are actually relative rankings. He uses the example of soil productivity, which can be 'measured' using a formula that takes into account a number of variables that affect soil productivity (e.g. soil depth, capacity for water retention, and nutrient content). These calculations result in an index that consists of numbers. Higher numbers indicate soils that are more productive and lower numbers indicate less productive soils.

"Such scales ... would allow us to say that a rating of 8 means more productive soils than a rating of 4 . They seldom, however, leave us in a position to say that a rating of 8 means soils twice as productive as a rating of 4. It is our inability to make this last statement that keeps such ratings from being true measurements. Instead, they are rankings." (Drennan 2009:223, emphasis his).

Although the phosphate results generated for this thesis turn out to be numbers, they - not unlike values calculated for soil productivity - represent the contribution of multiple variables. As such, the only way to compare them meaningfully is through their relative ranks. To find out if phosphate was a good indicator of occupational intensity, I compared it on a rank order basis to other inferred indicators of occupational intensity.

Like phosphate, it is similarly appropriate to compare site area and fish bone densities on this basis. Shell middens are difficult to separate into original deposition components and - as mentioned in previous chapters - it is impossible to know how frequently people returned to the same places and how long each occupation lasted. 'Site area' is an observation that does not necessarily represent an area of occupation. This variable refers to the cumulative area of multiple occupations over millennia. Although these areas are recorded in the field based on 


\section{M.A. Thesis - K. Carter; McMaster University - Anthropology}

probing of deposits to determine their archaeological extent (Cannon pers. comm.), the entire extent of deposits does not necessarily represent contemporaneous deposition. In this way, like phosphate, individual contributions to site area are not resolved, and comparison based on relative areas is appropriate. The same can be said for fish bone densities, which are calculated from multiple locations across a site. Individual episodes of deposition (i.e. the individual contribution to the density) cannot be resolved. Using rank order turns all three of these characteristics - which are present-day observations - into variables that can be compared meaningfully.

\subsection{Rank Order Correlations}

Non-parametric, rank order statistics do not take into account exact numerical values. Instead, ranks are applied and the differences between those ranks are used to assess whether a significant relationship exists between the variables. I used Spearman's rank order correlations to test whether one variable (phosphate) increased as another variable (FBD or site area) increased. The expectation was that there would be a positive relationship between fish bone density and phosphate, and site area and phosphate. Spearman's rank order correlation does not assume a normal distribution, nor does it rely on variables like means or standard deviations. It is therefore a highly and automatically robust statistic (Drennan 2009:228). This allows for a strong relative comparison considered best for this analysis.

\section{Spearman's Rank Order Correlations}

The initial analyses of phosphate and fish bone densities (Spearman's rho $=0.305, p=$ 0.102) and phosphate and site area (Spearman's rho $=0.072, p=0.385)$ did not show significant correlations. One possible contributing factor was the presence of sites with anomalous values for one or more of the variables. An initial visual assessment of the data showed that some sites did stand out from others for having low fish bone densities and/or small site areas, but also exhibiting very high phosphate values, contrary to expectations.

Based on the possibility that some sites were skewing the results of correlations, I determined the rank orderings of all sites for each individual variable - phosphate, fish bone density, and site area - and compared the differences between ranks to assess if any sites stood out as anomalous. Each site was numbered 1 through 19, from highest to lowest, except in the event of ties. Where ties occurred, I used the mean of the tied values instead (Drennan 
2009:225). For example, ElSx-10, ElTb-1, and ElSx-18 have the same phosphate value. If these three sites could be ordered, they would be 9, 10, and 11 in rank. Instead, they were all assigned a rank of 10 (the mean of 9,10 , and 11).

After ranks were assigned, I calculated the differences between ranks. Subtracting the rank ordering for one variable $(X)$ from the rank ordering of another $(Y)$ provides the difference (d) between rankings (Drennan 2009:225). Table 4.2 shows phosphate rankings $(X)$, fish bone density rankings $(Y)$, and the differences $(d)$ between them. Table 4.3 shows the same calculations for phosphate rankings $(X)$ and site area rankings $(Y)$. Differences in rank are variable among all these sites, but some clearly stood out.

Sites ElTb-2, ElTa-18 and ElTa-21 exhibit the largest differences in rank between phosphate and fish bone density. Sites ElTb-2, ElTa-21, and ElTa-3 show the largest differences in rank between phosphate and site area. Other sites, like EjSv-9, ElTb-1, EjSw-1, and ElSx-5 also exhibited differences that were somewhat large in relation to others.

TABLE 4.2. Differences between phosphate and fish bone density rankings.

\begin{tabular}{|c|c|c|c|c|c|c|}
\hline \multirow[b]{2}{*}{ Site } & \multirow[b]{2}{*}{ Site Name/Location } & \multirow{2}{*}{$\begin{array}{c}\text { Phosphate (ppb) } \\
X\end{array}$} & \multirow{2}{*}{$\begin{array}{c}\text { FBD (NISP/L) } \\
Y\end{array}$} & \multicolumn{2}{|c|}{ RANKINGS } & \multirow{2}{*}{$\begin{array}{c}\text { Difference } \\
\qquad d \\
\end{array}$} \\
\hline & & & & $x$ & $\boldsymbol{Y}$ & \\
\hline EjSv-9 & Taylor Bay & 3200 & 107.2 & 14 & 5 & 9 \\
\hline EITb-1 & Nulu & 3800 & 197.8 & 10 & 2 & 8 \\
\hline EISx-16 & Fougner Bay & 1400 & 66.8 & 17.5 & 11.5 & 6 \\
\hline EISx-3 & Kisameet & 3900 & 146.6 & 8 & 3 & 5 \\
\hline EjSw-1 & Cockmi & 3700 & 85.5 & 12 & 7 & 5 \\
\hline EISx-5 & Windsor Cove & 2800 & 68.5 & 15 & 10 & 5 \\
\hline EjSv-5 & Edna Matthewes Island & 1400 & 62 & 17.5 & 13 & 4.5 \\
\hline EISx-4 & Windsor Cove & 600 & 10.2 & 19 & 16 & 3 \\
\hline EIS $x-10$ & Fougner Bay & 3800 & 71.5 & 10 & 8 & 2 \\
\hline EISx-1 & Namu & 5400 & 114.9 & 5 & 4 & 1 \\
\hline EkSt-1 & Katit & 11400 & 320.9 & 1 & 1 & 0 \\
\hline ElTb-10 & McNaughton & 5200 & 98.7 & 6 & 6 & 0 \\
\hline EIS $x-18$ & Fougner Bay & 3800 & 66.9 & 10 & 11.5 & -1.5 \\
\hline EjSv-10 & Taylor Bay & 3400 & 34.7 & 13 & 16 & -3 \\
\hline EISx-8 & Fougner Bay & 1500 & 2.3 & 16 & 19 & -3 \\
\hline EITa-3 & Watt Bay & 6200 & 69.7 & 4 & 9 & -5 \\
\hline EITb-2 & Spitfire Channel & 7300 & 39 & 3 & 14 & -11 \\
\hline ElTa-18 & Kildidt Inlet & 5000 & 2.9 & 7 & 18 & -11 \\
\hline ElTa-21 & Kildidt Lagoon & 9700 & 3.7 & 2 & 17 & -15 \\
\hline
\end{tabular}


M.A. Thesis - K. Carter; McMaster University - Anthropology

TABLE 4.3. Differences between phosphate and site area rankings.

\begin{tabular}{|c|c|c|c|c|c|c|}
\hline \multirow[b]{2}{*}{ Site } & \multirow[b]{2}{*}{ Site Name/Location } & \multirow{2}{*}{$\begin{array}{c}\text { Phosphate (ppb) } \\
x \\
\end{array}$} & \multirow{2}{*}{$\begin{array}{c}\text { Site Area }\left(\mathrm{m}^{2}\right) \\
Y\end{array}$} & \multicolumn{2}{|c|}{ RANKINGS } & \multirow{2}{*}{$\begin{array}{c}\text { Difference } \\
\qquad d \\
\end{array}$} \\
\hline & & & & $x$ & $Y$ & \\
\hline$\overline{E j S w-1}$ & Cockmi & 3700 & 8590 & 12 & 1 & 11 \\
\hline EIS $x-5$ & Windsor Cove & 2800 & 2190 & 15 & 5 & 10 \\
\hline EjSv-9 & Taylor Bay & 3200 & 2040 & 14 & 6 & 8 \\
\hline EISX-8 & Fougner Bay & 1500 & 930 & 16 & 12 & 4 \\
\hline EjSv-5 & Edna Matthewes Island & 1400 & 530 & 17.5 & 14 & 3.5 \\
\hline EIS $x-1$ & Namu & 5400 & 8100 & 5 & 2 & 3 \\
\hline ElTb-10 & McNaughton & 5200 & 4500 & 6 & 3 & 3 \\
\hline EjSv-10 & Taylor Bay & 3400 & 1300 & 13 & 10 & 3 \\
\hline EIS $x-4$ & Windsor Cove & 600 & 140 & 19 & 16 & 3 \\
\hline EISx-16 & Fougner Bay & 1400 & 300 & 17.5 & 15 & 2.5 \\
\hline EISX-18 & Fougner Bay & 3800 & 1310 & 10 & 8.5 & 1.5 \\
\hline EIS $x-3$ & Kisameet & 3900 & 1630 & 8 & 7 & 1 \\
\hline ElTb-1 & Nulu & 3800 & 1120 & 10 & 11 & -1 \\
\hline ElTa-18 & Kildidt Inlet & 5000 & 1310 & 7 & 8.5 & -1.5 \\
\hline EkSt-1 & Katit & 11400 & 4260 & 1 & 4 & -3 \\
\hline EISx-10 & Fougner Bay & 3800 & 840 & 10 & 13 & -3 \\
\hline ElTa-21 & Kildidt Lagoon & 9700 & 130 & 2 & 17 & -15 \\
\hline ElTb-2 & Spitfire Channel & 7300 & 60 & 3 & 18 & -15 \\
\hline EITa-3 & Watt Bay & 6200 & 50 & 4 & 19 & -15 \\
\hline
\end{tabular}

There are limitations in the comparison of rank order relationships. Although there are ways to deal with rank ties, this type of comparison does not take into account that some subtly 'different' values may not actually be different. For example, site ElSx-18 is $1310 \mathrm{~m}^{2}$. Site EjSv10 is $1300 \mathrm{~m}^{2}$. A number of factors can affect the recorded spatial extent of a site. The difference in area between these sites is subtle and nearly the same. Despite this, EjSv-10 ends up ranked below ElSx-18. In this way, it is important to consider the impact differences in non-tied but largely similar values may have. To account for this, I subjectively decided to place emphasis only on the sites with the three largest differences for each comparison to tease out potential anomalies. I considered any other differences to be the result of expected variation in the sample. On this basis, ElTb-2, ElTa-18, ElTa-21 and ElTa-3 stand out as potential anomalies. All four have very high phosphate values for their size, and all happen to be associated with stone-wall fish traps in their immediate vicinity. To test their impact on the overall strength of correlation between occupational intensity indicators and phosphate values, these sites were removed from the dataset and the Spearman's rank order tests were re-run ${ }^{40}$.

\footnotetext{
${ }^{40}$ See section "Sites in Proximity to Fish Traps" for a discussion of these sites.
} 


\section{M.A. Thesis - K. Carter; McMaster University - Anthropology}

With these four sites removed, there is a strong and statistically significant rank order correlation between phosphate and fish bone densities (Spearman's rho $=0.795, p<0.001$ ). There is also a significant positive correlation between phosphate and site area (Spearman's rho $=0.683, p=0.002$ ). These results demonstrate that phosphate values generally relate positively to other indicators of occupational intensity, but also appear to vary according to the focus of site activity, specifically activities related to the mass harvest and processing of fish.

\subsection{Inferences \& Some Meaningful Comparisons}

The results of the above statistical comparisons establish phosphate as a useful independent measure of 'occupational intensity' - a reflection of the variable duration, scale and frequency of site occupations. This implies that phosphate should reflect the same residential patterns as do fish bone densities and site area. To explore this assumption, I drew categorical comparisons among these variables based on groupings established by Cannon for fish bone density and site area, and those that I observed and defined for phosphate.

Cannon took auger samples from a number of locations at each site (anywhere from one to six locations) and generated a site-level fish bone density. The numbers of fish bones and volumes of matrix used to standardize them can be found in Appendix D. Site-level densities can be found in Table 4.4 below, and in Table 1.1. Based on observed breaks in the data distribution, Cannon (2013a:27) found that sites fell into three groups: $>80$ bones/L, 50-80 bones/L and $<50$ bones/L (see Table 4.4). He also used site area to define two broad categories, either greater than or less than $800 \mathrm{~m}^{2}$.

I observed breaks in the distribution of phosphate values and defined groups. Phosphate values fall into three categories: > 5000 ppb, 2500-4500 ppb, and < $2000 \mathrm{ppb}$ (see Table 4.5). To create these groups, I visually examined and noted breaks in the distribution of phosphate values. These groups were based on the presence of major gaps in values. For example, while all but the lowest phosphate value in the lowest group fall within $100 \mathrm{ppb}$ of one another, the gap between this group and the next highest is $1300 \mathrm{ppb}$. Similarly, while all but the two lowest values in the middle group fall within $500 \mathrm{ppb}$ of one another, the gap between this group and the highest group is $1100 \mathrm{ppb}$. 


\section{M.A. Thesis - K. Carter; McMaster University - Anthropology}

TABLE 4.4. Sites tested in this study grouped based on FBD categories defined by Cannon. Data from Cannon (2000a:734, 2002:320, 2013a:24).

\begin{tabular}{|c|c|c|c|}
\hline & Site & & FBD (NISP/L) \\
\hline \multirow{7}{*}{$\begin{array}{c}>80 \\
\text { bones/L }\end{array}$} & EkSt-1 & (Katit) & 321 \\
\hline & EITb-1 & (Nulu) & 198 \\
\hline & EIS $x-3$ & (Kisameet) & 147 \\
\hline & EIS $x-1$ & (Namu) & 115 \\
\hline & EjSv-9 & & 107 \\
\hline & ElTb-10 & (McNaughton) & 99 \\
\hline & EjSw-1 & (Cockmi) & 86 \\
\hline \multirow{6}{*}{$\begin{array}{c}60-80 \\
\text { bones/L }\end{array}$} & EIS $x-10$ & & 72 \\
\hline & EITa-3 & & 70 \\
\hline & EIS $x-5$ & & 69 \\
\hline & EIS $x-18$ & & 67 \\
\hline & EISX-16 & & 67 \\
\hline & EjSv-5 & & 62 \\
\hline \multirow{6}{*}{$\begin{array}{c}<60 \\
\text { bones/L }\end{array}$} & EITb-2 & & 39 \\
\hline & EjSv-10 & & 35 \\
\hline & EIS $x-4$ & & 10 \\
\hline & EITa-21 & & 4 \\
\hline & EITa-18 & & 3 \\
\hline & EIS $x-8$ & & 2 \\
\hline
\end{tabular}

TABLE 4.5. Showing breaks in phosphate data.

\begin{tabular}{|c|c|c|c|}
\hline & Site & & Phosphate (ppb) \\
\hline \multirow{8}{*}{$>5000 \mathrm{ppb}$} & EkSt-1 & (Katit) & 11400 \\
\hline & ElTa-21 & & 9700 \\
\hline & ElTb-2 & & 7300 \\
\hline & EISx-1* & (Namu) & 6400 \\
\hline & EITa-3 & & 6200 \\
\hline & EISx-1 & (Namu) & 5400 \\
\hline & EITb-10 & (McNaughton) & 5200 \\
\hline & EITa-18 & & 5000 \\
\hline \multirow{8}{*}{$\begin{array}{c}2500- \\
4500 \mathrm{ppb}\end{array}$} & EISx-3 & (Kisameet) & 3900 \\
\hline & EISx-18 & & 3800 \\
\hline & EITb-1 & (Nulu) & 3800 \\
\hline & EISx-10 & & 3800 \\
\hline & EjSw-1 & (Cockmi) & 3700 \\
\hline & EjSv-10 & & 3400 \\
\hline & EjSv-9 & & 3200 \\
\hline & EISx-5 & & 2800 \\
\hline \multirow{4}{*}{$<2000 \mathrm{ppb}$} & EISx-8 & & 1500 \\
\hline & EjSv-5 & & 1400 \\
\hline & EISx-16 & & 1400 \\
\hline & EISx-4 & & 600 \\
\hline
\end{tabular}




\section{M.A. Thesis - K. Carter; McMaster University - Anthropology}

To facilitate comparisons, I designated each of the three categories derived for FBD and phosphate as 'high', 'moderate' and 'low', respective to their values (see Table 4.6). I considered sites that were $<800 \mathrm{~m}^{2}$, low to moderate in FBD, and low to moderate in phosphate to be consistent with expectations based on previously observed patterns. Sites that were $>800 \mathrm{~m}^{2}$, moderate to high in FBD, and moderate to high in phosphate were also consistent. With some exceptions, smaller sites that had low fish bone densities also exhibited low phosphate values. Larger sites with high fish bone densities are higher in phosphate.

TABLE 4.6. Categories used for categorical comparison. Site area and fish bone density categories were defined by Cannon (2013).

Categories for comparison:
\begin{tabular}{c|l|l|l|} 
Site Area & \multicolumn{1}{|c}{ Phosphate } & Fish Bone Density \\
\hline$>800 \mathrm{~m} 2$ & $>5000 \mathrm{ppb}$ & $>80$ bones/L & HIGH \\
$<800 \mathrm{~m} 2$ & $\begin{array}{l}2500-4500 \mathrm{ppb} \\
60-80 \text { bones/L }\end{array}$ & MOD \\
& $<2000 \mathrm{ppb}$ & $<60$ bones/L & LOW \\
\hline
\end{tabular}

Of the nineteen shell midden sites tested here, thirteen fit expectations of variable occupational intensity well. Sites ElTa-3, ElTb-2, ElTa-21, and ElTa-18, which stood out in the previous rank order comparisons, were distinguished again through categorical comparisons. Two further sites (ElSx-8 and EjSv-10) did not stand out using rank order comparisons, but seem anomalous based on this categorical analysis. They are explainable based on previous investigations.

\section{Sites that Fit Expectations}

Site ElSx-4 is a small site with low fish bone density and low phosphate. Sites ElSx-16 and EjSv-5 also exhibit low phosphate, which is consistent with Cannon's (2013a:27) description of these sites as camps. Site ElSx-10 is a base camp, being larger in area than some of the camp sites with similar FBD. It has a moderate phosphate level, which is consistent with this interpretation. Cannon (2013a:27) also describes ElSx-18 and ElSx-5 as similarly intensive base camps. They too have moderate phosphate values.

Larger village sites with moderate to high FBD, like Kisameet (ElSx-3), Katit (EkSt-1), McNaughton (ElTb-10), Namu (ElSx-1), and Cockmi (EjSw-1) all exhibit moderate to high phosphate values. Sites ElTb-1 and EjSv-9 also exhibit this same phosphate pattern, which is consistent with the overall form and inferred intensity of occupation at these sites. Cannon has 
described ElTb-1 as a spring/summer aggregate village and EjSv-9 as a possible base camp. Overall, the phosphate data fit with previously observed patterns and interpretations. They also highlight Cannon's (2013a:25) argument that these sites exist on a continuum of occupational intensity, rather than as strict site types, exhibited by the variability seen in phosphate results, even within the groupings I have observed and defined for use here.

\section{Sites Anomalous in their Size}

Site ElSx-8 covers a relatively large area, yet contains low densities of fish bone and is low in phosphate. Based on its recorded site area, the expectation would be that phosphate values and FBD should be relatively high. However, ElSx-8 consists of irregular patchy deposits distributed over a long linear area (see Figure 4.1). The form and nature of these deposits are inconsistent with intensive occupation (Cannon 2013a:27). Site ElSx-8 is actually a 'small' site spread irregularly over a wide area. The general relationship between phosphates, fish bone density, and site area that exists at other central coast sites therefore holds at ElSx-8 as well.
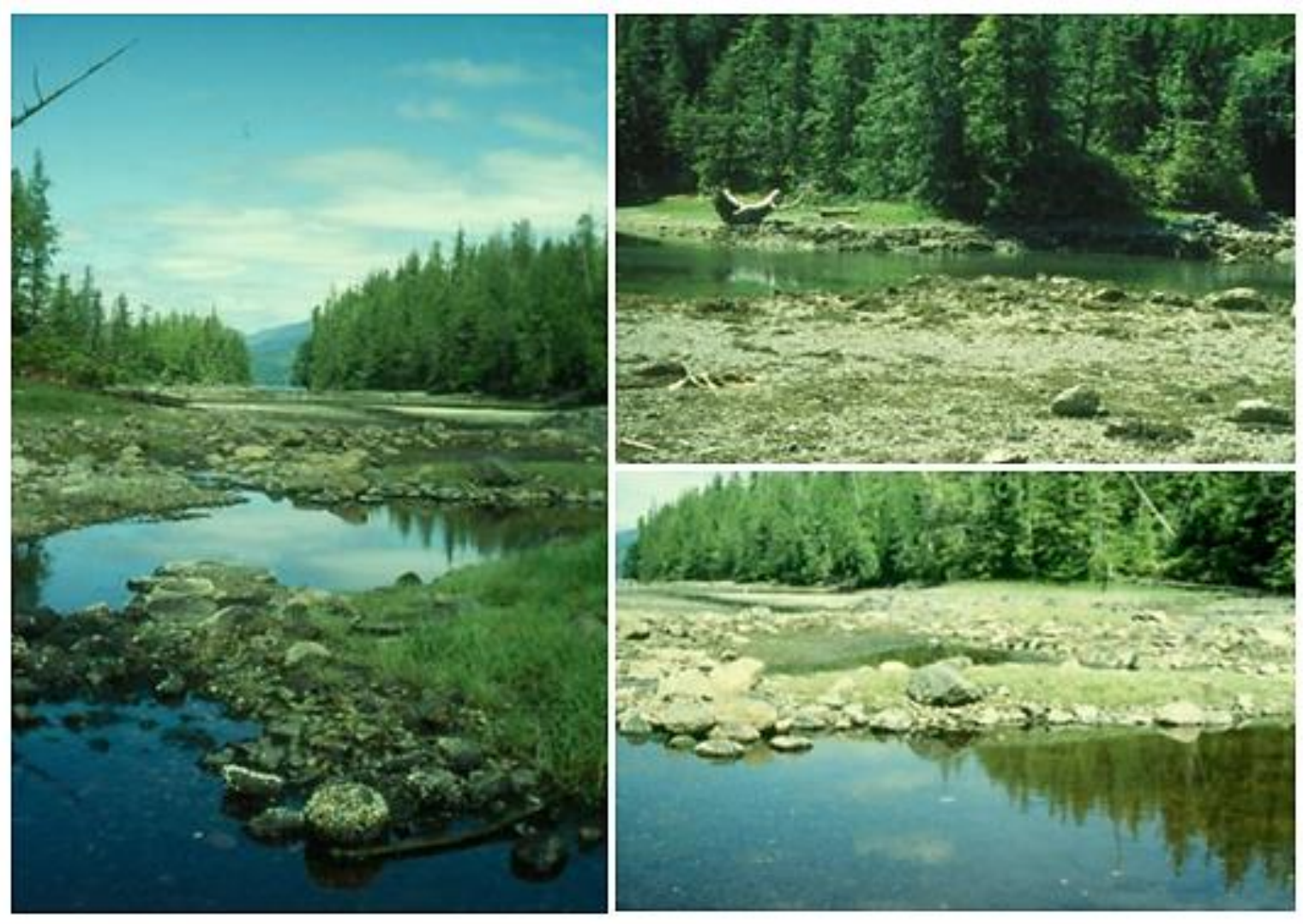

FIGURE 4.1. Photos of ElSx-8 supplied by Aubrey Cannon. 


\section{M.A. Thesis - K. Carter; McMaster University - Anthropology}

Site EjSv-10 is a medium area site located on a small island at the entrance to Taylor Bay. Cannon (2013a:32) notes that this site has a lower density of fish remains, despite its relatively large area. However, the deposition at EjSv-10 is unique in that the surface deposits at the western end of the site, that should have represented terminal occupation, are as old as the basal deposits at the eastern end of the site (Cannon 2013a:32).

"Rather than being a large midden, more akin to major residential sites, EjSv10 is a small camp, which developed at different times in two adjacent locations. The area of the site in each period of occupation was c. 500-700 square meters, which puts it in the same class as some of the other small camps" (Cannon 2013a:33).

In this way, EjSv-10 is also a small site, with low FBD and moderate phosphate values. It fits the pattern otherwise evident on the central coast of British Columbia.

\section{Sites in Proximity to Fish Traps}

- EITa-3 was distinguished based on rank order and categorical comparisons. Despite its small area, fish bone densities and phosphates are moderate and high respectively. This site is associated with a stone fish trap, which accounts for the high number of fish (mostly salmon) remains recovered there (Cannon 2013a:27). Phosphate values seem to speak to activity focus (i.e. salmon harvest), further supporting the interpretation of this site as a specialized salmon processing location.

- ElTb-2 is a small site that exhibits low densities of fish bone and high phosphate values. It was distinguished based on rank-order and categorical comparisons. It is located in proximity to a stone fish trap and an emphasis on fishing at this site was inferred on the basis of an unusual diversity of fish species represented at this site (Cannon 2013a:26). Based on the site's area and inferred emphasis on fishing, phosphates are best interpreted in support of ElTb-2 as a fish harvesting location, and minimally as a specialized encampment.

- ElTa-21 is distinguished based on both categorical and rank order comparisons. This small site is located on a narrow terrace overlooking Kildidt Lagoon and a nearby stone fish trap. It has a low FBD and a very high phosphate value. Cannon (2000a:734) noted 


\section{M.A. Thesis - K. Carter; McMaster University - Anthropology}

that the size of ElTa-21 and the nature of its deposits suggest that it was likely not a major place for habitation and was probably a seasonal fishing camp. The near absence of fish bones may relate to a similar absence of shell and possible preservation effects.

- ElTa-18 is distinguished based on rank order and categorical comparisons. It is a medium-sized site with a moderate fish bone density and a very high phosphate value. It is located across Kildidt Narrows from a fish trap. Its site area is also exaggerated by the inclusion of surface midden deposits that cover a long steep slope down to Kildidt Narrows from the smaller horizontal terrace where the main depth of cultural deposits is located (Cannon 2002:325). It is therefore is not appropriately described as a mediumsized site with respect to its available habitation area.

The basal deposits at ElTa-18 were dated to $9940 \pm 50$ years BP by Cannon (1998:16). McLaren et al. (2015:175), who also obtained an early date at this site, conducted a small-scale coring program in conjunction with excavations at ElTa-18 and suggested an intermittent pattern of site use. Therefore, the area of deposits is exaggerated relative to the area of occupation at any given time, similar to ElSx-8 and EjSv-10. ElTa-18 is actually a series of small site occupations imposed over a somewhat large area, which means that ElTa-18 fits the pattern expected for sites associated with fish traps: low fish bone density, very high phosphate, and small site area.

All four of these sites (ElTa-3, ElTb-2, ElTa-21, and ElTa-18) are associated with fish traps and seem to have their own unique phosphate signature. They are small in area, very high in phosphate, and, with one exception, low in fish bone density. At least at these four sites, there is a strong relationship between small sites associated with fish traps and phosphate concentrations. This is consistent with studies by Knudson and Frink (e.g. 2010b), who investigated the chemical signatures of contemporary fisheries activities. They showed that fish processing areas were high in a number of elements, phosphorus included. Small sites with phosphate signatures that seem anomalously high for their size may instead be indicative of intensive fish harvesting and processing activities.

In addition to these indicators, all of these sites also have low shell content. Figure 4.2 shows variation in the overall shell content of some of the sites examined in this study. The 


\section{M.A. Thesis - K. Carter; McMaster University - Anthropology}

abundance of shell is related to the intensity of shellfish gathering and variability in physical and chemical decay of shell in site deposits (Cannon et al. 2008).

Because shell content is partly a measure of the intensity of shell harvest and consumption, relatively less shell may indicate non-intensive harvest of shellfish. Or, that shellfish harvest was not a focus of activity at places with little shell. The four sites distinguished by phosphate and associated with fish traps have the lowest amount of shell in the Namu vicinity and adjacent outer coast tested by Cannon et al. (2008), although other sites tested in this thesis also have little shell. This suggests that shellfish harvest was not a focus of activity at these places and further speaks to the likelihood that they were specialized fish processing locations, rather than places where multiple economic activities were ongoing.

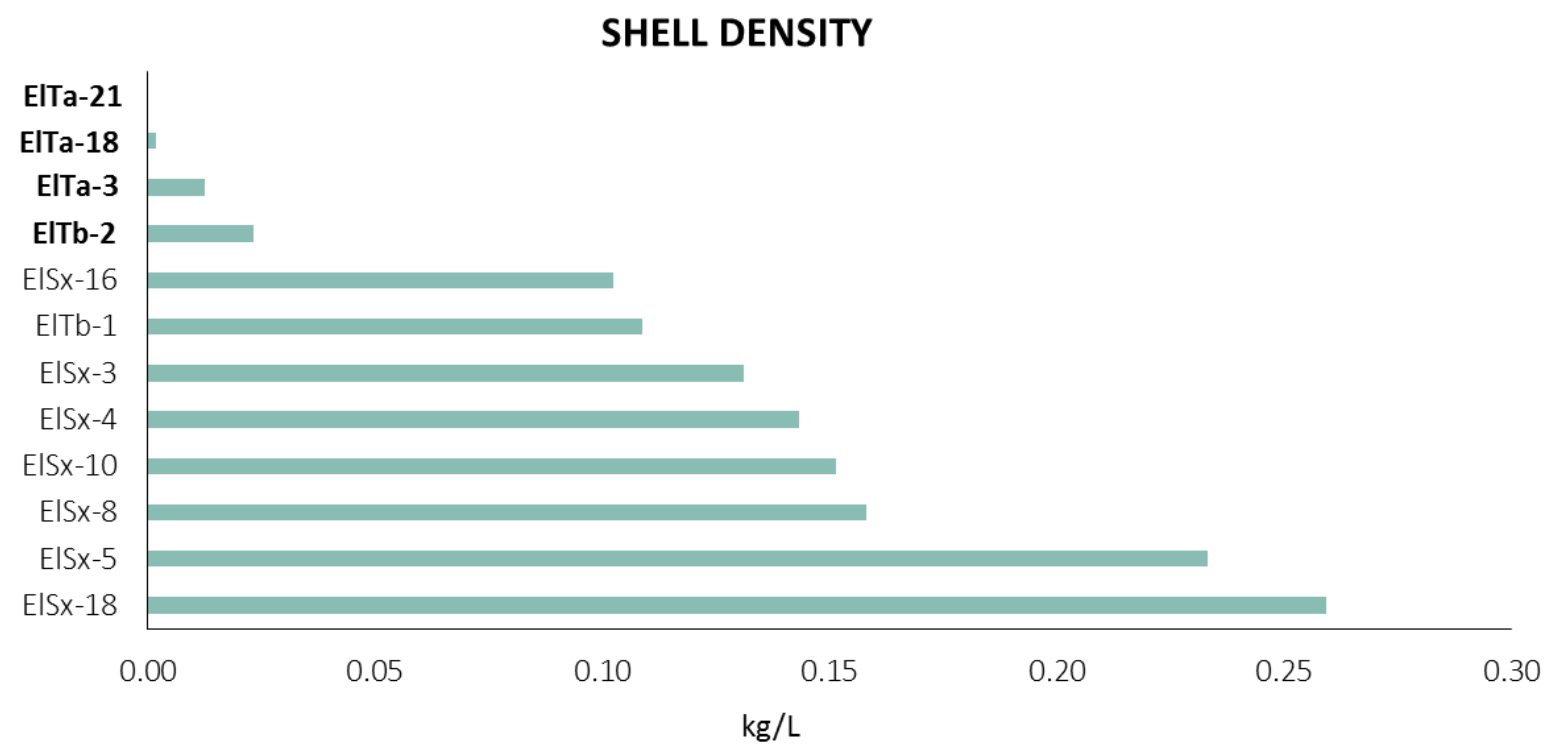

FIGURE 4.2. Showing variability in shell content at twelve central coast sites, with ElTa-21, ElTa-18, ElTa-3 and ElTb-2 highlighted. Values represent shell content in weight ( $\mathrm{kg})$, standardized by volume (L). Data are from Cannon et al. (2008).

Phosphate, therefore, can speak to some degree to the focus of activity at particular places. In addition, expecting small shell midden sites located in proximity to fish traps to have a high concentration of phosphate would be reasonable. Figure 4.3 represents all of the information expressed in the above results. It shows clearly the six sites that fall out of the otherwise somewhat linear relationship between fish bone densities and phosphate, demonstrating that as fish bone densities increase, phosphate increases. This chart provides a 
visualization for the relationship between fish bone density, site area, and phosphate as measures of occupational intensity, and how when combined they highlight both specialized sites and broader relationships among all.

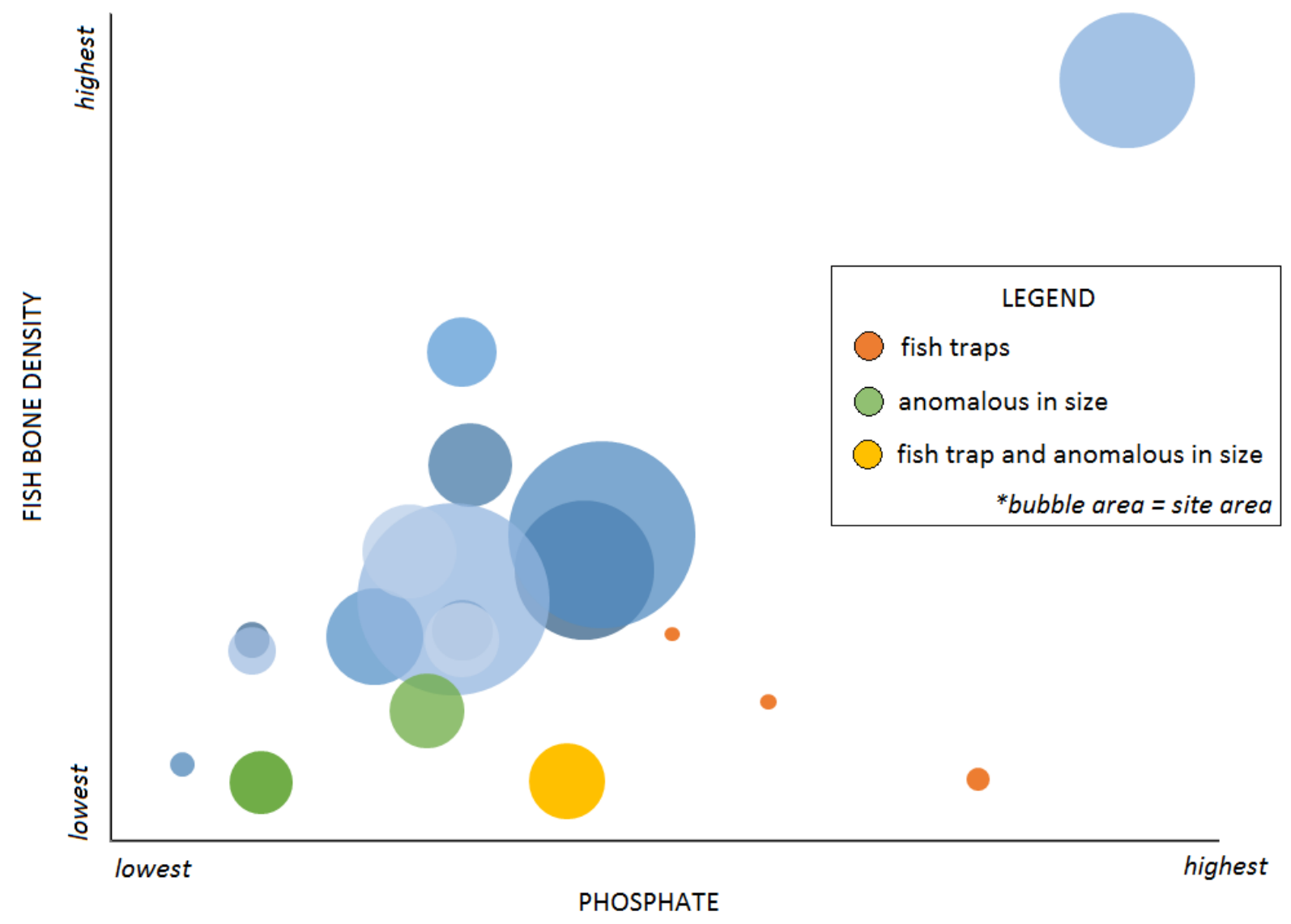

FIGURE 4.3. Showing the somewhat linear relationship between phosphate, fish bone density and site area, and how the anomalous sites fall out of the pattern in particular ways.

\section{Summary of Trends}

Overall, there is a range of variation in phosphate content among these nineteen shell midden sites that can be attributed in part to variability in occupational intensity based on strong and statistically significant Spearman's rank order correlations. Anomalously high phosphate values that do not fit this more general pattern show that phosphate may be a useful indicator of fish processing activities, which is further demonstrated by comparisons using 'high', 'moderate' and 'low' categories. With the exception of some anomalies distinguished through these analyses, which are easily explained, all sites tested here fit with patterns in settlement variety on the central coast of British Columbia. 


\section{M.A. Thesis - K. Carter; McMaster University - Anthropology}

Phosphate analysis helps to demonstrate further that some sites were occupied with a similar intensity, yet none is precisely the same. Phosphate, fish bone density, and site area are three independent measures of occupational intensity that, when considered together, not only show that these sites fall along a continuum, but also that their position on a scale results from different combinations of variables. To exemplify this, I took the rank ordering from the three variables considered here - phosphate, fish bone density, and site area - and summed them. I imagined that totaling all ranks would produce a value that simultaneously takes into account all three variables. I then ordered all of the sites based on the sum of their rankings to produce a sort of index of occupational intensity for these sites, demonstrating a continuum. Table 4.7 shows the results of this exercise.

TABLE 4.7. Rank orderings for phosphate, fish bone density, and site area, and their sums. Showing sites on a scale or continuum.

\begin{tabular}{|c|c|c|c|c|c|c|}
\hline \multirow[b]{2}{*}{ Site } & \multirow[b]{2}{*}{ Site Name/Location } & \multicolumn{3}{|c|}{ RANKINGS } & \multirow[b]{2}{*}{ Sum } & \\
\hline & & Phosphate & FBD & Site Area & & \\
\hline EkSt-1 & Katit & 1 & 1 & 4 & 6 & MORE \\
\hline EISx-1 & Namu & 5 & 4 & 2 & 11 & OCCUPIED \\
\hline ElTb-10 & McNaughton & 6 & 6 & 3 & 15 & \\
\hline EISx-3 & Kisameet & 8 & 3 & 7 & 18 & \\
\hline EjSw-1 & Cockmi & 12 & 7 & 1 & 20 & \\
\hline ElTb-1 & Nulu & 10 & 2 & 11 & 23 & \\
\hline EjSv-9 & Taylor Bay & 14 & 5 & 6 & 25 & \\
\hline EISx-5 & Windsor Cove & 15 & 10 & 5 & 30 & \\
\hline EISx-18 & Fougner Bay & 10 & 11.5 & 8.5 & 30 & \\
\hline EISx-10 & Fougner Bay & 10 & 8 & 13 & 31 & \\
\hline ElTa-3 & Watt Bay & 4 & 9 & 19 & 32 & \\
\hline EITa-18 & Kildidt Inlet & 7 & 18 & 8.5 & 33.5 & \\
\hline ElTb-2 & Spitfire Channel & 3 & 14 & 18 & 35 & \\
\hline EITa-21 & Kildidt Lagoon & 2 & 17 & 17 & 36 & \\
\hline EjSV-10 & Taylor Bay & 13 & 16 & 10 & 39 & \\
\hline EISx-16 & Fougner Bay & 17.5 & 11.5 & 15 & 44 & \\
\hline EjSv-5 & Edna Matthewes Island & 17.5 & 13 & 14 & 44.5 & \\
\hline EISX-8 & Fougner Bay & 16 & 19 & 12 & 47 & $\begin{array}{l}\text { LESS } \\
\text { INTENSIVELY }\end{array}$ \\
\hline EISx-4 & Windsor Cove & 19 & 16 & 16 & 51 & $\nabla$ OCCUPIED \\
\hline
\end{tabular}

This table shows three main things. First, it is clear that different combinations of these three variables result in the 'sum'. Different combinations of people and the frequency and 


\section{M.A. Thesis - K. Carter; McMaster University - Anthropology}

duration of their activity at these places produced the variables measured here, so it makes sense that this would be the case. Second, Cannon (2013a) notes that these sites fall along a continuum; this table provides one way to visualize this by combining phosphate with other variables. The colours assigned to the 'sum' column in this table are conditional on the values. The darker the colour the higher the value, and presumably the more intensively occupied the site. This gradient highlights how difficult it would be to determine where to draw distinctions, speaking to the fact that categories - which are useful tools for comparison - are descriptions. Third and last, this table shows that the overall trend evident when these three variables are combined fits well with what is understood about these sites. Longer-term villages line up on the "more intensively occupied' end of the scale, and shorter-term camp sites line up on the 'less intensively occupied' end. Phosphate can be usefully integrated with existing data, and has the further value of distinguishing some sites (e.g. fish processing locations).

\subsection{Representativeness: Beyond a Single Location}

All of the phosphate values used in this thesis come from a single auger location per site. Each auger location was chosen based on site characteristics and the likelihood that it was representative of the full site. Because I used one location to draw generalizations about entire sites, it was critical to assess this assumption. To do this I addressed the question: Is phosphate generally representative of site conditions if it is only measured from a single location?

To answer this question, I made one overarching assumption: if the auger location chosen for analysis were representative of site conditions, then phosphate concentrations from that location would also be representative of the site's phosphate conditions. This reasoning is based in part on the ubiquity of phosphate relative to other sample components that are more volatile in their distribution. If comparatively more volatile sample constituents are representative of full site conditions, it follows that phosphate, as a more ubiquitous component of the same sample, would also be representative.

Fish bones are an example of an auger sample component that have greater potential variability in their patterns of deposition, as the product of individual events of processing, consumption and discard, compared to phosphates, which are potentially deposited more evenly as a result of their association with a wider range and greater number of contributory events. I hypothesized that if the fish bone density at a single auger location is the same as the fish bone 


\section{M.A. Thesis - K. Carter; McMaster University - Anthropology}

density for the whole site based on multiple auger locations, then that specific location likely represents the whole site's deposition. Multiple auger locations are sufficient for generalizing about a whole site (Cannon 2000a), so if a single location reflects those multiple locations, then it too is representative of the whole site.

To test this hypothesis, Spearman's rank order correlations were run to compare the fish bone densities from individual auger locations ${ }^{41}$ with the fish bone densities generated for entire sites ('site-level') $)^{42}$. Results show a strong and statistically significant correlation between fish bone densities from individual auger locations and site-level fish bone densities (Spearman's rho $=0.763, p=0.003$ ). This suggests that the auger locations chosen for this analysis represent full site conditions.

So fish bone densities from a single auger location are representative of the fish bone densities for an entire site. It follows that phosphate is also representative given its ubiquity relative to fish bone. This initial test on its own suggests that phosphate from a single auger location represents the site's phosphate conditions, but I chose to explore this question further using phosphate data. I hypothesized that if phosphate from an auger location were representative of site conditions, then the strongest positive correlations should come from comparisons with the site-level fish bone densities. Similarly, if phosphate from a single auger location were not representative of the site's phosphate conditions, then it would be most strongly correlated with its exact sample context - that is, the precise $7-15 \mathrm{~cm}$ vertical interval from which it was derived.

To test this, I used 'site-level' fish bone densities as a proxy for the whole site, fish bone densities calculated for individual auger locations, and fish bone densities from specific 7-15 cm samples used in the phosphate analysis. If phosphate is representative of whole site conditions, it should correlate most strongly with site-level fish bone densities (those generated for the whole site), and least strongly with fish bone densities from corresponding contexts (the same $7-15 \mathrm{~cm}$ sample from which phosphate was derived). The correlation between phosphate values and fish bone densities from a single auger location should fall somewhere in-between.

\footnotetext{
41 These fish bone densities are unpublished. Aubrey Cannon provided the data used to generate these values.

${ }^{42}$ At some sites, only one auger location was sampled. These sites were not included in this analysis.
} 


\section{M.A. Thesis - K. Carter; McMaster University - Anthropology}

To explore this hypothesis, three Spearman's rank order tests were conducted ${ }^{43}$. The first test compared site-level fish bone densities with phosphate. Results show a strong and statistically significant relationship between these variables (Spearman's rho $=0.785, p<0.001$ ). The second test compared fish bone densities from individual auger locations with phosphate. Results also show a strong and statistically significant relationship between these variables (Spearman's rho $=0.753, p=0.001$ ). The third test compared phosphate and fish bone densities using densities generated from exact sample contexts.

Before presenting results of the third test, further explanations are warranted. As mentioned, I tested individual auger samples representing approximately 7-15 cms of deposits for phosphate. For the most part, I tested every sample from each auger location for phosphate, but in some cases I tested every other sample in the interest of time. This meant that I could compare phosphate and fish bone densities in their 'exact contexts'. In the test for individual auger locations, phosphate values representing the auger location were compared against the fish bone density generated using all samples from that location; in this 'exact context' test, I generated a new fish bone density using only the 7-15 cm samples that were tested for their phosphate values. Again, because I used every sample to generate phosphate values in some cases and every other sample in others, the fish bone density dataset used for this third test consists in part of values generated using every other sample, and in part from values generated using every sample. Results from this test show a strong and statistically significant correlation between phosphate and fish bone density (Spearman's rho $=0.726, p=0.001$ ).

Table 4.8 lists all of results from the three tests presented above. As expected, site-level comparisons between phosphate and fish bone density exhibited the strongest correlation. This suggests that phosphate values from single auger locations are representative of full site conditions. The specific auger location comparison produced comparatively weaker correlations (although they are still strong and significant); the exact sample correlation was even weaker than the auger location correlation.

\footnotetext{
${ }^{43}$ The anomalous fish trap sites were not included in this analysis.
} 


\section{M.A. Thesis - K. Carter; McMaster University - Anthropology}

TABLE 4.8. Results of three Spearman's rank order tests.

\begin{tabular}{|l|rr|}
\hline & Spearman's rho & Sig. $(p)$ \\
\hline Site-level comparisons & $\mathbf{0 . 7 8 5}$ & $<\mathbf{0 . 0 0 1}$ \\
Auger-averaged comparisons & $\mathbf{0 . 7 3 4}$ & $\mathbf{0 . 0 0 1}$ \\
Exact context comparisons & $\mathbf{0 . 7 2 6}$ & $\mathbf{0 . 0 0 1}$ \\
\hline
\end{tabular}

\section{Representativeness of Every Other Sample}

To further evaluate the reliability of results based on every other auger sample from particular auger locations, I generated new phosphate averages and fish bone densities using every other sample from all sites. If phosphate from every other sample is as representative as phosphate from every sample, this correlation should be as strong as the correlation between phosphate and auger-averaged fish bone densities. Results from this test show a strong and statistically significant correlation between phosphate and fish bone density (Spearman's rho = $0.729, p=0.001$ ). This result is similar to the auger location comparisons (refer to Table 4.8). These results show that using every other sample (when samples are taken at approximately 7-15 $\mathrm{cm}$ intervals) is sufficient for drawing conclusions about relative phosphate values. It is not necessary to test every sampled context to generate comparable site level results, though that may be necessary to identify significant horizontal variability in phosphate values.

\subsection{Generalizability: Beyond British Columbia's Central Coast}

I had an opportunity to examine matrix from two shell midden sites located in Prince Rupert Harbour (PRH), on the north coast of British Columbia (see Figure 4.4). This allowed me to address the question of whether this method can be extended beyond the sites tested from the central coast. Although both the central coast and the northern coast are similar in their environments, there was precedent for this test based on differences in observed fish bone densities between central and northern coast sites (Brewster and Martindale 2011). This test was conducted to examine whether the phosphate values from these two PRH sites fit with expectations based on the results from the central coast. That is, would a smaller, potentially less intensively occupied PRH site exhibiting a lower FBD produce a lower phosphate value than a site presumed to be more intensively occupied and exhibiting a higher FBD? Although both PRH sites exhibited low to moderate FBD by central coast standards, both are considered to be intensively occupied residential settlements (i.e. villages). Although based on only three samples per site, the site with the lower fish bone density exhibited a phosphate value of $2200 \mathrm{ppb}$. The 


\section{M.A. Thesis - K. Carter; McMaster University - Anthropology}

site with the higher fish bone density exhibited a value of $5300 \mathrm{ppb}$, which falls within the range found for village sites on the central coast.

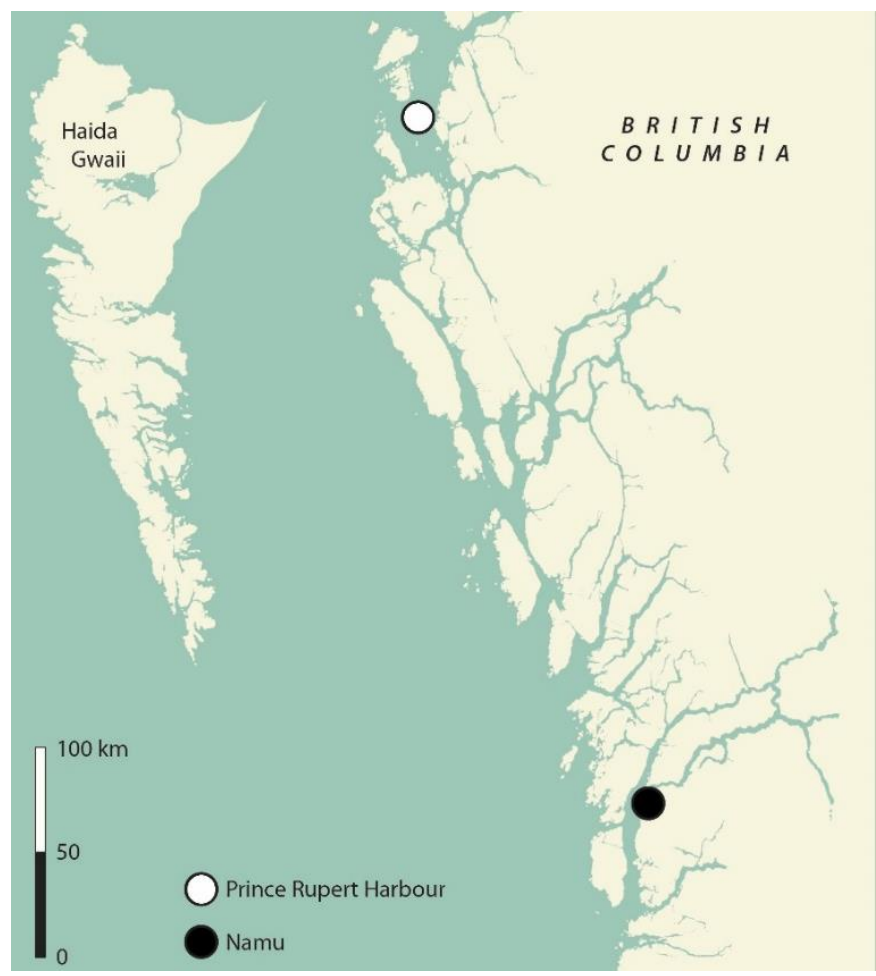

FIGURE 4.4. Map showing location of the PRH in relation to Namu and the central coast. Map by Mary Lynn Tobiasz

These results show that this application of phosphate analysis can be extended beyond the central coast. It may be applicable to all shell midden sites, but certainly seems generally applicable on the Pacific Northwest Coast.

\subsection{XRF Analysis}

In addition to colorimetry, XRF analysis was conducted on sediments from nine of the nineteen sites tested in this thesis (Carter et al. 2015). To do this, an ITRAX core scanner, which is instrumentation for high-resolution core analysis, was used to analyze small quantities of fine $(<0.6 \mathrm{~mm})$ shell midden matrix. Samples were tested for multiple elements, phosphorus included. One of the goals of this study was to compare high-resolution XRF against lowresolution colorimetry as two different measures of $\mathrm{P}$ content.

\section{Methods}

To do this, a sample tray that contained a row of $1 \mathrm{~cm}$ by $1 \mathrm{~cm}$ cubes separated by plastic partitions, was used. I filled each cube with sediment from a unique sample, which resulted in an 
approximation of a sediment profile for each site tested (see Figure 4.5). This involved dampening existing dried sediments with distilled water in order to pack them into the cubes. The auger location tested at each of these sites was the same location I tested for phosphate.

After the sediment trays were filled, they were run through the ITRAX core scanner. The scanner was set to collect data at 500 micron increments, which meant that approximately 20 readings were taken per sample. Each reading is the total number of counts taken at an integration time of 15 seconds (i.e. the reading is the number of counts detected over a period of 15 seconds). These data were not calibrated to a concentration. Instead, the counts - which provide the relative proportions of elements in a sample were used. A photograph was also documented alongside the chemical data. Data for multiple sites and numerous elements were produced overnight.

Because the sample tray consists of plastic partitions, it was first important to remove any data generated for the plastic that separates each sediment sample. Fortunately, these data are very high-resolution and patterns in dips and peaks are easily discernible. I was readily able to distinguish the chemical signature of the plastic from the shell midden matrix, usually by a stark drop in the concentration of numerous elements. This allowed me to segregate the approximately 20 readings per sediment sample, discard the irrelevant plastic data, and generate an average value for each element for each sample.

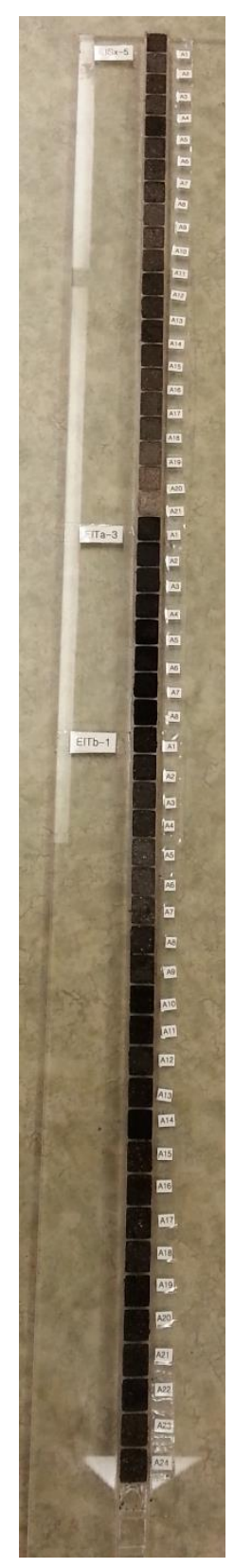

FIGURE 4.5. Example of filled XRF sample tray.

\section{Colorimetry vs. XRF}

As a way to simultaneously assess phosphorus (total) as a measure of occupational intensity and to compare low-resolution colorimetry with high-resolution XRF, some of the phosphate results I generated for this thesis were compared against phosphorus values generated using the core scanner. XRF generated phosphorus values were also compared against fish bone densities. Figure 4.6 shows how closely these three variables - FBD, phosphate, and phosphorus - correspond at ElSx-3, as an example. This demonstrates the wider validity of using phosphorus 
concentrations to measure the form and intensity of site occupation, and the parallels between these high- and low-resolution techniques.

\section{EISx-3, Auger C}

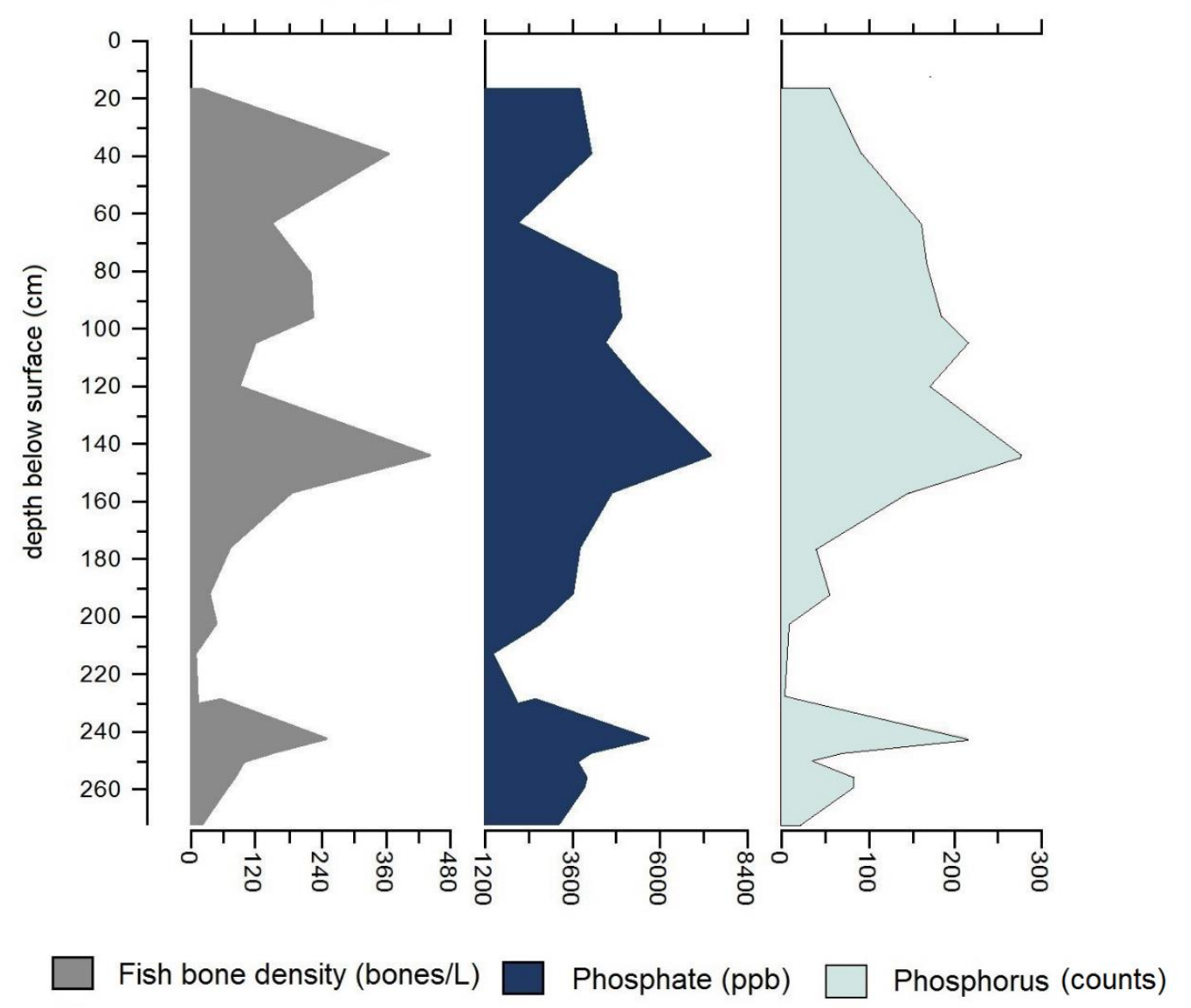

FIGURE 4.6. Showing comparisons between fish bone density, phosphate (colorimeter) and phosphorus (XRF) at ElSx-3.

Although measures of total $\mathrm{P}$ and available $\mathrm{P}$ are not necessarily comparable (see Chapter 2), in this case the XRF measures of total $P$ reflect patterns in the available $P$ measurements obtained using the colorimeter. The profiles do not exactly mirror one another, but they are very close. This suggests that perhaps most of the phosphorus that exists in these shell midden samples is available P. The mineral component of shell midden sites is small and consists mostly of inputs from fire cracked rock or sand brought onto the site and incorporated into the midden, and even those components are not high in mineral phosphate. The concentration of available $\mathrm{P}$ matches well with the relative proportions of phosphorus measured using XRF. 


\section{M.A. Thesis - K. Carter; McMaster University - Anthropology}

From these preliminary XRF analyses, available $\mathrm{P}$ measures and total $\mathrm{P}$ measures seem comparable. Because the profiles are not exact, site-level comparisons probably remain most appropriate, though trends through both vertical profiles are also clear. Although I cannot make meaningful inferences about the dips and peaks in these profiles based on these comparisons, at a minimum their general correspondence shows that these two methods are comparable in this context.

\subsection{Results Summary}

The following lists the results of this thesis:

- Phosphate is a useful indicator of occupational intensity, established by strong and statistically significant Spearman's rank order correlations between phosphate values and other indicators (FBD and site area) used to infer occupational intensity.

- Rank-order comparisons show that phosphate, in addition to being a useful measure of occupational intensity, can distinguish fish processing locations.

- Large sites with high fish bone densities that were likely occupied for long periods by many people exhibit high phosphate values.

- Small sites with low fish bone densities that were likely occupied for short periods by few people exhibit low phosphate values.

- Sites that do not fit this pattern are explainable. For example, shell midden sites associated with fish traps are small, often have relatively few fish remains, but are very high in phosphate.

- Phosphate values from a single auger location are generally representative of a whole site's phosphate conditions.

- This application is generalizable beyond the sites tested here. It may be applicable to all shell middens, but is minimally applicable to other parts of the NWC.

- XRF analysis of shell midden sediments for total phosphorus shows a strong correspondence with phosphate values obtained using the colorimeter, suggesting that (1) both measurements of $\mathrm{P}$ reflect patterns in occupational intensity, (2) the P content of shell midden sites - at least in this study - consists largely of available P, and (3) that these two measures are likely comparable in this context, though that may not always be the case at other types of sites.

These results indicate that phosphate analysis is useful for characterizing the residential nature of shell midden sites and addressing activity foci at intensive fish processing locations. They also show that a single auger location is sufficient for drawing generalizations about a whole site, and 
M.A. Thesis - K. Carter; McMaster University - Anthropology

that this application has the potential to be extended beyond the sites that were the focus of this study. 


\section{M.A. Thesis - K. Carter; McMaster University - Anthropology}

\section{Chapter 5: Discussion and Conclusions}

In this chapter, I examine the findings of this study within the broader context of central coast settlement patterns, landscape studies, and chemical analysis of sediments in archaeological contexts. My goal is to integrate phosphate studies within a broader central coast narrative. I then move to discuss some specific implications this thesis has for archaeology as a discipline, highlighting what I think are its unique contributions and limitations. I then turn to discuss my thoughts on future research on this topic, before presenting the overall conclusions.

\subsection{Within a Settlement Narrative for the British Columbian Central Coast}

Settlement patterns on the Northwest Coast have been derived largely from ethnography, which projects into the past an understanding of economic/procurement strategies based on seasonal movements (e.g. Ames 1981; Fladmark 1975). In this particular instance, it means a cycle of large winter village occupations, followed by spring-summer aggregations, and movement finally to small fish procurement locations before returning to the winter village. Put so simply, this interpretation obscures the variability and particularity of different histories and circumstances that may result in a different - or at least more complex - story.

Writing and researching the more complex reality of Northwest Coast settlement history is challenging because shell midden stratigraphy is complicated and logistical constraints on traditional excavations in remote contexts such as the central coast are not conducive to multisite investigations (Cannon 2000a:726; Hester and Conover 1970), yet an understanding of regional settlement history cannot be based on investigations of a single midden site. This was the initial basis for Cannon's core and auger sampling approach to regional investigation (Cannon 2000a, 2000b, 2002, 2013a). This approach has facilitated the development of an initial narrative of central coast settlement. My goal was to assess the potential role of phosphate analysis in further supporting and clarifying this emerging overview of regional settlement history.

Bailey (2007:205) talks about the visibility and invisibility of activities to archaeologists based on repetition and revisiting or reuse. The material manifestations of everyday life are only visible if sufficient deposition exists to see them; that is, if people were active at a particular place often and intensively enough that their materials have accumulated to the point of 


\section{M.A. Thesis - K. Carter; McMaster University - Anthropology}

remaining persistent. Persistence and visibly have been implicit themes of this thesis. In order for phosphate values to mean anything in relation to a real, regional pattern, I am relying directly on the persistence of phosphate as an archaeological indicator of past activities, and people's persistent use of places in the landscape and the accumulation of phosphate that results. The results of my analysis show that phosphate, fish bone density, and site area are mutually valid indicators of a continuum of occupational intensity.

The coarse resolution of shell midden deposits precludes any discussion of individuals or recognizable social groupings. The palimpsest nature of shell middens means that deposits are physically blended, and that "individuals ... very quickly disappear from view" (Bailey 2008:21). Instead of finely resolved chronologies or groupings, we instead work with obscured accumulations. Bailey (2008:21) writes about different ways archaeologists have aimed to "put the individual back into the archaeological picture". He notes that one strategy is to ignore the problem of coarse resolution and continue to explain patterns in the archaeological record in a more traditional way. But "this is scarcely sustainable when the mismatch between such concepts and the scale and resolution of the archaeological record ... has become so obvious" (Bailey 2008:21). Instead, it is key to remember and recognize that individuals, who may not be explicitly in the forefront of investigations, are paramount to the patterning we observe at a large scale - however accumulated and fragmented. In Bailey’s (e.g. 2008:22) view, these accumulations offer a different perspective.

In Chapter 1, I mentioned that time-perspectivism has influenced my understanding of shell middens and phosphate analysis at this scale. The idea that different scales bring into focus different patterns and processes is exemplified by conceptualizing phosphate through comparison with a more finely resolved context where it is typically employed. As mentioned in Chapter 1, the phosphate analysis I employed in this thesis can be compared to activity area analysis at a single component site. Archaeologists chemically analyzing house floors, for example, identify concentrated areas of use from which they can draw inferences about how people lived in a space. In the same way, a regional analysis of multiple shell midden sites across a large and variable landscape renders concentrated use areas visible. Where this approach differs from others is in its ability to speak to variability in the magnitude of those concentrations. Rather than addressing spatial distribution and areal extent alone, we are also able to see the degree to which 


\section{M.A. Thesis - K. Carter; McMaster University - Anthropology}

people used certain site locations in relation to others. We see the intensity of activity at different places, within a patterned, regional distribution and a much larger spatial landscape.

The narrative for the central coast of British Columbia is one of variability and continuity in subsistence economies, and relationships among places over at least 7000 years at a local and regional scale. The economic history of the Namu site is easily the most archaeologically wellknown within the region (e.g. Cannon 1991; Carlson 1979, 1996). Multiple lines of evidence have come together to show a particular history of periodic decline and recovery of the local Namu salmon fishery, that peaked at 6000-4000 years ago, reached maximum decline by 2000 years ago, and returned to levels of peak productivity once again by 1000 cal years BP (Cannon 1991). Further evidence shows that this pattern of decline and subsequent recovery of the Namu salmon fishery was not simply an overall trend. Rather, the fishery experienced periodic failures during its trending decline, although it is impossible to know the precise details of these failures or their eventful nature due to a coarse temporal resolution (Cannon et al. 2011:61).

In addition to fisheries studies, scholars have also investigated patterns in shellfish harvest (Cannon and Burchell 2009; Cannon et al. 2008), the distribution of red ochre (MacDonald et al. 2011; Macdonald et al. 2013), and the presence or absence of parasites and their implications for health (Bathurst 2005a, 2005b). These ubiquitous residues of daily activity, along with fish bone densities and now, phosphate, speak as reliable indicators of occupational intensity and the seasonal and locational focus on particular activities. They also add specific nuances to patterns of site occupation.

At the very least, phosphate corresponds with what was already known about the history of these places. Overall, winter and seasonal villages contain high concentrations of phosphate and small, short-term encampments contain low levels. But because phosphate is an indicator of numerous activities simultaneously, it can also speak to trends in occupational intensity regardless of fish bone deposition. This means that it can potentially add some clarity to the relative relationships between sites that may remain slightly ambiguous.

Sites ElSx-5, ElSx-10 and ElSx-18 all contain fish bones, but the density and the variety of the remains are relatively low. All of these sites have been described as similarly intensive residential base-camps (Cannon 2013a:27). The pattern or variability in their relative intensities, however, could not be determined (Cannon 2013a:26). Sites ElSx-10 and ElSx-18 exhibit similar 


\section{M.A. Thesis - K. Carter; McMaster University - Anthropology}

phosphate values, whereas ElSx-5 is slightly higher. When all three variables - phosphate, fish bone density, and site area - are integrated, they show that all three of these sites fall in the middle of the overall range of variability in the intensity of site occupations on the central coast, and were occupied to essentially the same degree of intensity. In this case, integrating phosphate does not necessarily add clarity as much as it adds additional evidence that these sites were occupied to a similar degree of intensity. Phosphate is an ideal indicator of occupational intensity in that it simultaneously represents multiple activities, though it cannot be used to identify any one activity in particular.

The exception to that statement would be at fish-trap sites, where phosphate is clearly useful as an indicator of a specific single activity: fish harvest and processing. It is not that phosphate 'detects' fish processing activities at this scale, since clearly fish were harvested and processed at a number of places explored here; it is that phosphate can readily distinguish the strong chemical signal of this single, focussed activity. Phosphate has added to an understanding of these sites that was not previously clear based on fish bone deposition alone. Site ElTa-18, for example, is located across Kildidt Narrows from a stone fish trap. Although the trap is proximate to the site, it is not in direct association (i.e. not located on the site's beachfront) and it remained unclear what the focus of activity was at this location, except to say that the nature of its deposits were inconsistent with intensive occupation (Cannon 2013:27). Similarly, ElTa-21 is in the general proximity of a fish trap feature, but the site matrix yielded almost no direct evidence of fishing in the form of fish bones. The phosphate signature, however, was consistent with intensive fish processing at this location. Site ElTb-2 did contain a wide variety of fish remains, yet a low density and was inferred as a likely candidate for fish harvest and processing, but the phosphate results point even more strongly toward this interpretation.

Regional settlement patterns on the central coast of British Columbia are variable. They contain a range of different occupations that materially represent varying numbers of people who lived at these places and conducted different activities for different amounts of time. By considering all of these variables together as 'occupational intensity,' a pattern that sees sites existing on a continuum from more intensively occupied to less intensively occupied emerges. Phosphate integrates well with existing indicators of this pattern. Phosphate can also help to 


\section{M.A. Thesis - K. Carter; McMaster University - Anthropology}

clarify, to some degree, ambiguities in the relative occupational intensity among different places based on other indicators.

\subsection{Implications for the Discipline}

I have anchored this discussion around three main themes: (1) shell midden archaeology, (2) the archaeological analysis of soil or sediment, and (3) regional settlement studies. I review the ways my thesis aligns with each of these themes as presented in the background, assess results in light of this background, and outline some of the contributions of this thesis.

\section{What Phosphate means for Shell Midden Archaeology}

Archaeologists studying shell midden sites explore a range of interests globally. Some of these interests include understanding site formation through sampling and building chronologies, and determining the nature of occupations, including site function and the scale of activity (Ascher 1959; Bailey 1977; Lyman 1991; Martindale et al. 2009; Stein et al. 2003). One critical debate seems to surround the build-up or formation of middens: to what extent did they result from residential activities over time? Or were they ritual constructions? (Luby et al. 2006; Marquardt 2010). Phosphate analysis, combined with other indicators, can contribute to this debate by helping to determine the relative scale of a range of residential activity.

As results from the central coast of British Columbia show, phosphate analysis is useful for distinguishing between long-term residential sites and shorter-term occupations. It can also distinguish fish processing locations. Although all of the sites tested here are considered residential, theoretically phosphate analysis should help to resolve that question in places where the residential nature of shell middens remains unclear. If shell midden sites are the product of incremental build-up over time from day-to-day activity, they should consistently contain higher phosphate levels than midden sites deliberated 'constructed' for ritual or other purposes over shorter periods of time.

Further, although the results presented here are site-level averages that speak to broader, long-term trends, I did observe some variability in phosphate profiles from the top of some sites to the bottom. This means that the application of phosphate analysis at shell middens could speak to questions concerning variability in site use over time, though I did not specifically explore this potential. 


\section{M.A. Thesis - K. Carter; McMaster University - Anthropology}

On the Northwest Coast, there is a mixed history of approaches to shell middens. My thesis aligns most closely with those who conduct detailed analysis of midden components and deposition. Results from this thesis show that phosphate analysis as applied on the central coast of British Columbia can be extended to other parts of the Northwest Coast. From the Prince Rupert harbour study I referred to in Chapter 4, there is basis to suggest that sites in other regions of the NWC may fall within the same range of values as the central coast. Although the study was very small in scale it clearly showed that larger villages located in two different NWC settings show a similar phosphate signature. The same is true for smaller camps. I expect this same pattern to exist regardless of where sites are located on the NWC. However, this conjecture is based only on two sites from a region other than the central coast, so my ability to speak to this idea is currently limited.

In sum, some implications of phosphate analysis for the study of shell midden sites include (1) addressing the residential nature of site occupation, potentially in contribution to a larger global debate on the formation of middens as ritual or residential, (2) the potential for intra-site temporal analysis, and (3) providing an additional archaeological indicator for understanding shell midden formation. The specific methods I have employed here are additionally appealing in their economic accessibility.

Phosphate values, as a proxy for human activity, provide a way to evaluate independently patterns that are indicated in other data. In combination with those data, results of phosphate analysis can help to resolve ambiguities in site use patterns. Phosphate analysis can also stand alone as a relatively reliable indicator of intensities of residential occupation. The sampling requirements for phosphate are also even less than those needed for the recovery and analysis of fish bone. A regional program of site sampling for phosphate analysis potentially could sample an even larger number of sites in much less time than was required for Cannon's program of core and auger sampling.

Phosphate also seems to be more widely comparable than other indicators. In Prince Rupert Harbour, for example, sites expected to have similar fish bone densities to sites on the central coast do not. Regardless of differences in fish bone deposition or preservation, fish bone densities between these two regions cannot be compared on the same scale (Brewster and Martindale 2011). The relative occupational intensity of PRH sites cannot be compared with that 


\section{M.A. Thesis - K. Carter; McMaster University - Anthropology}

of sites on the central coast based on fish bone densities, but they can be meaningfully compared using phosphate. Although very preliminary, the results presented in this thesis show that something about the nature of phosphate - whether it be its relative ubiquity or preservation speaks more widely and potentially more consistently to occupational intensity than do fish bone densities. Again, phosphate cannot speak to specific trends in regional fisheries, it is a good proxy for human activity, and a broadly applicable indicator of occupational intensity.

\section{What Phosphate Analysis of Shell Midden Sediments means for Archaeological Soil and Sediment Chemistry}

Although some of the earliest applications of sediment chemistry in archaeological research were conducted at shell midden or mound sites (Cook and Treganza 1947), the chemical analysis of midden sediments remains rare relative to its application in other contexts, and it is certainly not routine. By taking an established archaeological technique and applying it to a different context, I have aimed to extend the premise of archaeological sediment chemistry with an application at a scale of analysis that is not typically investigated.

Soil and sediment chemistry in archaeology traditionally involve horizontal, spatial analyses, usually of activity areas. Archaeologists have typically continued on the same trajectories of investigation, applying higher-resolution techniques to answer these questions in new ways. The basis of this thesis rests necessarily in the well-established context for British Columbia's central coast. In that way, these results cannot stand alone. However, following from the works of people like Parnell et al. (2001) and Schlezinger and Howes (2000), I think the results of this thesis show that applying soil/sediment chemistry in archaeology does not have to be just about activity area analysis or site detection or delineation. And perhaps phosphate may be useful for characterizing sites in this context on its own. Extending this particular project to include more sites would be one way of testing this. I would be comfortable drawing generalizations about less well-known sites based on phosphate if values fell within the range for the central coast. I am unsure whether the results of this thesis are generalizable beyond shell middens, but I do think a similar technique could be explored for other types of sites.

In terms of phosphorus (P) analysis specifically, this thesis speaks further to the debate surrounding the efficacy of available $\mathrm{P}$ versus total $\mathrm{P}$ for archaeologists. As I mentioned in Chapter 2, it seems that no particular measure of phosphorus is inherently appropriate for 


\section{M.A. Thesis - K. Carter; McMaster University - Anthropology}

archaeologists. In this study, available phosphate values matched patterns evident in other indicators (i.e. site area and fish bone density). This suggests that the decision to test for available $\mathrm{P}$ or total $\mathrm{P}$ should be an educated choice based on resolution and cost-effectiveness. Comparisons between XRF measures of phosphorus and colorimetric measures of phosphate suggest that the majority of $\mathrm{P}$ in shell midden sediments is likely available $\mathrm{P}$. The methods employed here may be limited in their ability to answer different kinds of questions, but they proved appropriate for this specific application.

\section{How Phosphate speaks to Long-term Histories and Regional Archaeologies}

Although the scale of this analysis precludes a discussion of individual site histories, this thesis has aligned with broader goals in settlement pattern research that aim to understand regional systems. It adds to understanding of NWC settlement patterning by contributing to ongoing work that has integrated a well-known site into a broader context, characterized by locally specific variability and long-term continuities (Cannon 2000a, 2000b, 2002; Cannon et al. 2011; Cannon 2013a). Phosphate has proven to be another variable indicator of residential activity that is a cumulative representation of multiple activities (see Table 2.2). In this thesis, phosphate was usefully integrated with existing indicators, further demonstrating a patterned settlement

distribution consisting of a range of variably intense occupations. Results of this thesis align well with the current state of knowledge surrounding settlement patterns on the central coast of British Columbia. They have also provided a basis to consider future research on this topic and application.

\subsection{Future Directions}

Based on my experience with this research and the implications it has for the discipline as a whole, I have developed a number of potential areas for future inquiry. My goal in this section is to highlight these, and to explain why I think they would be worthy of investigation.

First, based on this study, I think that a broader regional analysis of phosphate on the Northwest Coast would make an important contribution to existing understandings both of NWC settlements systems, and to how archaeologists utilize elemental analysis. In the same way that areas of concentrated use becomes clear as we 'zoom out', patterns at an even larger scale may become evident if we make even broader comparisons. We may have the potential to see the 


\section{M.A. Thesis - K. Carter; McMaster University - Anthropology}

long-term manifestation of a system of settlement that speaks to both change and continuity at a coast-wide scale.

Second, I think multi-element analysis of fine-fraction shell midden material would be a promising application. Although not presented as a component of this thesis, preliminary XRF results show variability in elements other than phosphorus at shell midden sites. There is the potential for addressing variability in vertical profiles, in addition to spatial variation among sites. Multi-element analysis could add a temporal dimension that is lacking from the phosphate analysis applied in this thesis. Based on the association of multiple elements with different activities and archaeological contexts, it may also have the potential to speak to activity foci in more detail than the research conducted here by bringing together numerous potential elemental indicators. Elements other than phosphorus that are ubiquitously distributed within shell middens may serve as further indicators of occupational intensity. Potassium, for example, is a good contender, as wood ash - a large component of shell middens - is high in potassium. In these ways, considering a variety of elements may simultaneously show site-specific trends, and regional residential patterns, potentially at multiple scales.

Third, I think this application could be extended to other coastal areas. Based on the initial results of PRH sites analysis, further application in that region would help to resolve the issue of variability in the intensity and focus of site occupation, which is at present unclear based on the fish bone densities observed in auger samples (Brewster and Martindale 2011).

Fourth and last, I think the comparison of elemental concentrations with other microarchaeological indicators would be interesting. Densities of fish bone provided a comparative indicator for phosphate. Presumably, other relatively small and ubiquitous indicators would provide new insights and, as Cannon (2013a:33) has noted, parasite eggs and small granules of ochre show this potential. Cannon (e.g. 2011) has characterized the nature of fisheries on the central coast of British Columbia by investigating vertebrate faunal content in numerous ways. This has not only provided insights into variability in the intensity of different occupations, but also into more site-specific fisheries (Cannon et al. 2011). This simultaneously speaks to a larger settlement pattern, and a localized economy. Densities of plant remains, if preserved, would hypothetically paint a similar picture based on a different aspect of subsistence. This would 


\section{M.A. Thesis - K. Carter; McMaster University - Anthropology}

provide an expanded depiction of patterns in subsistence settlement, and potentially another indicator of occupational intensity.

Phytoliths in particular, if they preserve in NWC shell middens, may show interesting patterns. I bring up phytoliths because they are siliceous and probably do not experience the same fate as other plant remains, like seeds, which tend to preserve better in shell middens when they are charred or in wet environments (Cannon pers. comm.). Phyoliths are also very small so they - like fish bones and phosphates - would likely be distributed ubiquitously. Although some plants are important foodstuffs, others are not. That said, a site-level comparison using relative abundances of standardized phytolith densities might show interesting patterns, especially under the assumption that sites would experience similar deposition of non-food-related plant materials during times when sites were not being used, and otherwise in shell middens which are entirely the product of human deposition.

Further, because plants have a tendency to 'interact' with people (i.e. some encroach on open land unless they are managed, while others are important foods), comparing vertical phytolith distributions against heightened or lowered elemental concentrations may be useful for identifying intermittencies in occupation. It would be interesting to see if plotting 'non-food' abundances against 'food-plant' abundances on a vertical profile would correlate in any way with elevated or depleted elements.

\subsection{Conclusions}

This thesis has explored phosphate as an independent indicator of occupational intensity at shell midden sites on the central coast of British Columbia. At the outset, my expectation was that phosphate, as a good general indicator of human occupation and an abundant and persistent element, would reflect the nature of occupations at different locations. A further expectation was that differences in occupational intensity accounted for the particular distribution of phosphate values seen among the sites tested here.

Initial correlations established that variability in phosphate is a measure of occupational intensity, and subsequent analyses drew more meaningful comparisons between phosphate, fish bone density, and site area. This showed that phosphate could distinguish a range of occupations, from long-term residential sites to short-term camps. Phosphate is therefore a useful means to 


\section{M.A. Thesis - K. Carter; McMaster University - Anthropology}

characterize residential shell midden sites based on their relationship on a scale. It can also distinguish fish trap sites, and add interpretive clarification to some sites. These site-level characterizations translate into a comparative description of sites that is useful for understanding settlement patterns on a regional scale. Phosphate was effectively integrated with existing data. This application also shows promise for extension beyond the central coast of British Columbia.

In this thesis, I have aimed to contribute to the discourse surrounding shell midden content and form. Results have served to add phosphate to the list of indicators that are useful for informing about histories of shell midden formation. I have also aimed to add to the literature on the elemental analysis of archaeological soils and sediments. By investigating phosphate at shell middens for regional comparisons, this work has extended an existing technique to a new context, where archaeologists have yet to employ it routinely. Using a low-cost analytical method means that this application can easily be applied in other contexts. 


\section{References Cited}

Abrahams, Peter W., Jane A. Entwistle, and R.A. Dodgshon

2010 The Ben Lawers Historic Landscape Project: Simultaneous Multi-element Analysis of Former Settlement and Arable Soils by X-ray Fluorescence Spectrometry. Journal of Archaeological Method and Theory 17(3): 231-248.

Acheson, Steven R.

1995 In the Wake of the Iron People: a Case for Changing Settlement Strategies Among the Kunghit Haida. The Journal of the Royal Anthropological Institute 1(2): 273-299.

Ames, Kenneth M.

1981 The Evolution of Social Ranking on the Northwest Coast of North America. American Antiquity 46(4): 789-805.

Arrhenius, Olaf

1934 Fosfathalten I Skanska Jordar. Sveriges Geologiska Undersokning 28: 1-30.

1963 Investigation of soil from old Indian sites. Ethnos 28: 122-136.

Ascher, Robert

1959 A Prehistoric Population Estimate Using Midden Analysis and Two Population Models. Southwestern Journal of Anthropology 15(2): 168-178.

\section{Bailey, Geoff N.}

1977 Shell Mounds, Shell Middens, and Raised Beaches in the Cape York Peninsula. Mankind 11: $132-143$.

2007 Time perspectives, palimpsests and the archaeology of time. Journal of Anthropological Archaeology 26(2): 198-223.

2008 Time Perspectivism: Origins and Consequences. In Time in Archaeology: Time Perspectivism Revisited, edited by Simon Holdaway and LuAnn Wandsnider, pp. 14-30. The University of Utah Press, Salt Lake City. 
Bailey, Kathleen M.

2006 Effects of ground-water flow on soil chemistry, nutrient availability and plant species distributions in four New York State fens. Ph.D. Dissertation, Cornell University.

Bathurst, Rhonda R.

2005a Archaeological evidence of intestinal parasites from coastal shell middens. Journal of Archaeological Science 32: 115-123.

2005b Health and Settlement Implications of Parasites from Pacific Northwest Coast Archaeological Sites. Ph.D. Dissertation, Department of Anthropology, McMaster University.

Bethell, Philip, and Ian Máté

1989 The Use of Soil Phosphate Analysis in Archaeology: A Critique. In Scientific Analysis in Archaeology and its Interpretation, edited by Julian Henderson, pp. 1-29. Oxford University Committee for Archaeology.

Birch, Jennifer

2016 Interpreting Iroquoian site structure through geophysical prospection and soil chemistry: Insights from a coalescent community in Ontario, Canada. Journal of Archaeological Science: Reports 8: 102-111.

Brewster, Natalie, and Andrew Martindale

2011 An Archaeological History of Holocene Fish Use in the Dundas Island Group, British Columbia. In The Archaeology of North Pacific Fisheries, edited by Madonna L. Moss and Aubrey Cannon, pp. 247-264. University of Alaska Press, Fairbanks.

Brück, Joanna, and Melissa Goodman

1999 Making places in the prehistoric world: themes in settlement archaeology. UCL Press.

Burchell, Meghan

2013 Shellfish Harvest on the Coast of British Columbia: The Archaeology of Settlement and Subsistence through High-resolution Stable Isotope Analysis and Sclerochronology. Ph.D. Dissertation, Department of Anthropology, McMaster University. 
Burchell, Meghan, Aubrey Cannon, Nadine Hallmann, Henry P. Schwarcz, and Bernd R. Schöne

2013a Refining Estimates for the season of shellfish collection on the pacific northwest coast: Applying high-resolution stable oxygen isotope analysis and sclerochronology. Archaeometry 55(2): 258-276.

Burchell, Meghan, Aubrey Cannon, Nadine Hallmann, Henry P. Schwarcz, and Bernd R. Schöne

$2013 \mathrm{~b}$ Inter-site variability in the season of shell fish collection on the central coast of British Columbia. Journal of Archaeological Science 40(1): 626-636.

Cannon, Aubrey

1991 The Economic Prehistory of Namu. Archaeology Press, Simon Fraser University, Burnaby, British Columbia.

1997 Permit Report - 1996-115: Investigations at ElSx-3, ElSx-6, ElSx-8, ElSx-10, ElSx-16, ElTa-3, ElTa-25, and EkSx-12. Report on file with Archaeology Branch. Victoria, BC.

1998a Contingency and Agency in the Growth of Northwest Coast Maritime Economies. Arctic Anthropology 35(1): 57-67.

1998b Permit Report - 1997-163: Investigations at ElSx-4, ElSx-5, ElSx-6, ElSx-8, ElSx-10, ElSx-18, ElTa-18, ElTa-21, ElTb-1, and ElTb-2. Vol. 1. Report on file with Archaeology Branch. Victoria, BC.

2000a Assessing Variability in Northwest Coast Salmon and Herring Fisheries: Bucket-Auger Sampling of Shell Midden Sites on the Central Coast of British Columbia. Journal of Archaeological Science 27(8): 725-737.

2000b Settlement and Sea-Levels on the Central Coast of British Columbia: Evidence from Shell Midden Cores. American Antiquity 65(1): 67-77.

2002 Sacred Power and Seasonal Settlement on the Central Northwest Coast. In Beyond Foraging and Collecting, edited by Ben Fitzhugh and Junko Habu, pp. 311-338. Kluwer Academic/Plenum Publishers, New York, Boston, Dordrecht, London, Moscow.

2013a Revealing the Hidden Dimensions of Pacific Northwest Coast Shell Middens. In Shell Energy: Mollusc Shells as Coastal Resources, edited by Geoffrey N. Bailey, Karen Hardy, and Abdoulaye Camara, pp. 21-34. Oxbow Books.

2013b Report on Archaeological Investigations in Rivers Inlet under Permit 2005-204. Report on file with Archaeology Branch. Victoria, BC. 
Cannon, Aubrey, and Meghan Burchell

2009 Clam growth-stage profiles as a measure of harvest intensity and resource management on the central coast of British Columbia. Journal of Archaeological Science 36(4): 10501060 .

2016 Reconciling oxygen isotope sclerochronology with interpretations of millennia of seasonal shellfish collection on the Pacific Northwest Coast. Quaternary International. In press.

Cannon, Aubrey, Meghan Burchell, and Rhonda R. Bathurst

2008 Trends and Strategies in Shellfish Gathering on the Pacific Northwest Coast of North America. In Early Human Impact on Megamolluscs, edited by A. Antczak and R. Cipriani, pp. 7-22. Archaeopress, Oxford, England.

Cannon, Aubrey, and Dongya Y. Yang

2009 Early Storage and Sedentism on the Pacific Northwest Coast: Ancient DNA Analysis of Salmon Remains from Namu, British Columbia. American Antiquity 71(1): 123-140.

Cannon, A., Dongya Y. Yang, and Camilla Speller

2011 Site-specific Salmon Fisheries on the Central Coast of British Columbia. In The Archaeology of North Pacific Fisheries, edited by Madonna L. Moss and Aubrey Cannon, pp. 57-74. University of Alaska Press, Fairbanks.

Carlson, Roy L

1979 The Early Period on the Central Coast of British Columbia. Canadian Journal of Archaeology 3(3): 211-228.

1996 Early Namu. In Early Human Occupation in British Columbia, edited by Roy L. Carlson and Luke Dala Bona, pp. 83-102. UBC Press.

Carter, Kari, Aubrey Cannon, and Eduard Reinhardt

2015 High-resolution XRF analysis of bulk shell midden matrix using a core scanner. Poster presented at the 47th Annual Meeting of the Canadian Archaeological Association, St. John's.

Conover, Kathryn Jane

1972 Archaeological Sampling at Namu: A Problem in Settlement Reconstruction. Ph.D. Dissertation, Department of Anthropology, University of Colorado. 
1978 Matrix Analyses. In Studies in Bella Bella Prehistory, edited by James J. Hester and Sarah M. Nelson, pp. 67-100. Department of Archaeology, Simon Fraser University, Burnaby, British Columbia.

Conway, J.S.

1983 An investigation of soil phosphorus distribution within occupation deposits from a Romano-British hut group. Journal of Archaeological Science 10(2): 117-128.

Cook, S.R., A.S. Clarke, and M.G. Fulford

2005 Soil geochemistry and detection of early Roman precious metal and copper alloy working at the Roman town of Calleva Atrebatum (Silchester, Hampshire, UK). Journal of Archaeological Science 32(5): 805-812.

Cook, S.F.

1946 A Reconsideration of Shellmounds with Respect to Population and Nutrition. Society for American Archaeology 12(1): 50-53.

Cook, S.F. F, and A.E. Treganza

1948 The Quantitative Investigation of Aboriginal Sites: Complete Excavation with Physical and Archaeological Analysis of a Single Mound. Society for American Archaeology 13(4): 287-297.

Cook, S.F., and R.F. Heizer

1965 Studies on the Chemical Analysis of Archaeological Sites. University of California Press.

Cook, S.F., and A.E. Treganza

1947 The Quantitative Investigation of Aboriginal Sites: Comparative Physical and Chemical Analysis of Two California Indian Mounds. Society for American Archaeology 13(2): 135-141.

Dalan, Rinita A., John M. Musser Jr., and Julie K. Stein

1992 Geophysical Exploration of the Shell Midden. In Deciphering a Shell Midden, edited by Julie K. Stein, pp. 43-58. Academic Press, San Diego. 
Dietz, Eugene F.

1957 Phosphorus Accumulation in Soil of an Indian Habitation Site. American Antiquity 22(4): 405-409.

Drennan, Robert D.

2009 Statistics for Archaeologists: A Common Sense Approach. 2nd ed. Springer, New York.

Eberl, Markus, Marco Álvarez, and Richard E. Terry

2012 Chemical Signatures of Middens at a Late Classic Maya Residential Complex, Guatemala. Geoarchaeology 27(5): 426-440.

Eddy, Frank W., and Harold E. Dregne

1964 Soil Tests on Alluvial and Archaeological Deposits, Navajo Reservoir District. El Palacio 71(4): 5-21.

Eidt, Robert C.

1973 A Rapid Chemical Field Test for Archaeological Site Surveying. American Antiquity 38(2): 206-210.

1977 Detection and Examination of Anthrosols by Phosphate Analysis. Science 197: 13271333.

Entwistle, Jane A., Peter W. Abrahams, and R.A. Dodgshon

2000 The Geoarchaeological Significance and Spatial Variability of a Range of Physical and Chemical Soil Properties from a Former Habitation Site, Isle of Skye. Journal of Archaeological Science 27(4): 287-303.

Fladmark, Knut

1975 A Paleoecological Model for Northwest Coast Prehistory. Mercury Series, No. 43. National Museums of Canada, Ottawa, Ontario, Canada.

Ford, Pamela J.

1989 Archaeological and Ethnographic Correlates of Seasonality: Problems and Solutions on the Northwest Coast. Canadian Journal of Archaeology 13: 133-150. 
1992 Interpreting the Grain Size Distributions of Archaeological Shell. In Deciphering a Shell Midden, pp. 283-324. Academic Press, San Diego.

Frink, Liam, and Kelly J. Knudson

2010 Using Ethnoarchaeology and Soils Chemistry to Examine Fisheries on the Arctic Alaskan Coast. North American Archaeologist 31(2): 221-247.

Gifford, E.W.

1916 Composition of California Shellmounds. University of California Publications in American Archaeology and Ethnology 12(1): 1-29.

Greenlee, Diana M.

1992 Effects of Recovery Techinques and Postdepositional Environment on Archaeological Wood Charcoal Assemblages. In Deciphering a Shell Midden, edited by Julie K. Stein, pp. 261-289. Academic Press, San Diego.

\section{Griffith, M. A.}

1980 A pedological investigation of an archaeological site in Ontario, Canada, I. An examination of the soils in and adjacent to a former village. Geoderma 24(4): 327-336.

Ham, Leonard C.

1982 Seasonality, Shell Midden Layers, and Coast Salish Subsistence at the Crescent Beach Site, DgRr 1. Ph.D. Dissertation, Department of Anthropology and Sociology, University of British Columbia.

Hamond, F.W.

1983 Phosphate analysis of archaeological sediments. In Landscape Archaeology in Ireland, edited by T. Reeves-Smyth and F. Hamond, pp. 47-80. BAR British Series 116, Oxford, UK.

Hester, James J.

1978 The Bella Bella Prehistory Project. In Studies in Bella Bella Prehistory, edited by James J Hester and Sarah M Nelson, pp. 1-11. Department of Archaeology, Simon Fraser University, Burnaby, British Columbia. 


\section{M.A. Thesis - K. Carter; McMaster University - Anthropology}

Hester, James J., and Kathryn Jane Conover

1970 Ecological Sampling of Middens on the Northwest Coast. Northwest Anthropological Research Notes 4(2): 137-151.

Holliday, Vance T.

2004 Soils in Archaeological Research. Oxford University Press, New York.

Holliday, Vance T., and William G. Gartner

2007 Methods of soil P analysis in archaeology. Journal of Archaeological Science 34(2): 301333.

Holliday, Vance T., Julie K. Stein, and William G. Gartner

2004 Appendix 3: Variability of Soil Lab Procedures and Results. In Soils in Archaeological Research, edited by Vance T. Holliday, pp. 363-374. Oxford University Press, Oxford, England.

Johnson, A.H.

1956 Examination of Soil from Corrimony Chambered Cairn, Glenurquhardt, Inverness-shire, with special reference to phosphate content. Appendix II. In Excavations in passagegraves and ring cairns of the Clava Group. Proceedings of the Society of Antiquaries of Scotland, edited by S. Piggott, pp. 173-207.

Kanthilatha, Nelum, William Boyd, and Nigel Chang

2014 Multi-element characterization of archaeological floors at the prehistoric archaeological sites at Ban Non Wat and Nong Hua Raet in Northeast Thailand. Quaternary International: 1-13. In press.

Knudson, Kelly J., and Liam Frink

2010a Soil chemical signatures of a historic sod house: Activity area analysis of an arctic semisubterranean structure on Nelson Island, Alaska. Archaeological and Anthropological Sciences 2(4): 265-282.

2010b Ethnoarchaeological analysis of Arctic fish processing: chemical characterization of soils on Nelson Island, Alaska. Journal of Archaeological Science 37(4): 769-783. 
Knudson, Kelly J., Lisa Frink, Brian W. Hoffman, and T.Douglas Price

2004 Chemical characterization of Arctic soils: activity area analysis in contemporary Yup'ik fish camps using ICP-AES. Journal of Archaeological Science 31(4): 443-456.

Kowalewski, Stephen A.

2008 Regional settlement pattern studies. Journal of Archaeological Research 16(3): 225-285.

LaMotte

2016 SMART3 Colorimeter: Operator's Manual

Lorch, W.

1940 Die siedlungsgeographische phosphatemethode. Die Naturwissenschaften 28: 633-640.

Lubos, Carolin, Stefan Dreibrodt, and André Bahr

2016 Analysing spatio-temporal patterns of archaeological soils and sediments by comparing pXRF and different ICP-OES extraction methods. Journal of Archaeological Science: Reports 9: 44-53.

Luby, Edward M., Clayton D. Drescher, and Kent G. Lightfoot

2006 Shell Mounds and Mounded Landscapes in the San Francisco Bay Area: An Integrated Approach. The Journal of Island and Coastal Archaeology 1(2): 191-214.

Lyman, R.L.

1991 Prehistory of the Oregon Coast: The Effects of Excavation Strategies and Assemblage Size on Archaeological Inquiry. Academic Press, San Diego.

MacDonald, B. L., R.G.V Hancock, A. Cannon, F. McNeill, R. Reimer, and A. Pidruczny

2013 Elemental Analysis of Ochre Outcrops in Southern British Columbia, Canada. Archaeometry 55(6): 1020-1033.

MacDonald, Brandi Lee

2008 Ochre Procurement and Distribution on the Central Coast of British Columbia. M.A. Thesis, Department of Anthropology, McMaster University. 
MacDonald, Brandi Lee, Ronald G.V. Hancock, Aubrey Cannon, and Alice Pidruczny

2011 Geochemical Characterization of Ochre from Central Coastal British Columbia, Canada. Journal of Archaeological Science 38: 3620-3630.

Manzanilla, Linda, and Luis Barba

1990 The Study of Activities in Classic Households: Two Case Studies from Coba and Teotihuacan. Ancient Mesoamerica 1: 41-49.

Marquardt, William H.

2010 Shell Mounds in the Southeast: Middens, Monuments, Temple Mounds, Rings, or Works? American Antiquity 75(3): 551-570.

Martindale, Andrew, Bryn Letham, Duncan McLaren, David Archer, Meghan Burchell, and Bernd R. Schöne

2009 Mapping of subsurface shell midden components through percussion coring: examples from the Dundas Islands. Journal of Archaeological Science 36(7): 1565-1575.

McKechnie, Iain M.P.

2013 An Archaeology of Food and Settlement on the Northwest Coast. University of British Columbia.

McLaren, Duncan, Farid Rahemtulla, Gitla (Elroy White), and Daryl Fedje

2015 Prerogatives, Sea Level, and the Strength of Persistent Places: Archaeological Evidence for Long-Term Occupation of the Central Coast of British Columbia. BC Studies 187: $155-191$.

Middleton, W. D.

2004 Identifying chemical activity residues on prehistoric house floors: A methodology and rationale for multi-elemental characterization of a mild acid extract of anthropogenic sediments. Archaeometry 46(1): 47-65. 
Middleton, William D, Luis Barba, Alessandra Pecci, James H Burton, Agustin Ortiz, Laura Salvini, and Roberto Rodriguez Suárez

2010 The Study of Archaeological Floors: Methodological Proposal for the Analysis of Anthropogenic Residues by Spot Tests, ICP-OES, and GC-MS. Journal of Archaeological Method and Theory 17: 183-208.

Middleton, William D., and Douglas T. Price

1996 Identification of Activity Areas by Multi-element Characterization of Sediments from Modern and Archaeological House Floors Using Inductively Coupled Plasma-atomic Emission Spectroscopy. Journal of Archaeological Science 23(5): 673-687.

Milek, Karen B., and Howell M. Roberts

2013 Integrated geoarchaeological methods for the determination of site activity areas: A study of a Viking Age house in Reykjavik, Iceland. Journal of Archaeological Science 40(4): 1845-1865.

Misarti, Nicole, Bruce P. Finney, and Herbert Maschner

2011 Reconstructing site organization in the eastern Aleutian Islands, Alaska using multielement chemical analysis of soils. Journal of Archaeological Science 38(7): 1441-1455.

Mitchell, Donald H, and Leland Donald

1988 Archaeology and the Study of Northwest Coast Economies. In Research in Economic Anthropology, Supplement 3: Prehistoric Economies on the Northwest Coast, edited by Issac Barry, pp. 293-351. JAI Press Inc., Greenwich, CT.

Moss, Madonna L.

1984 Phosphate analysis of archaeological sites, Admiralty Island, southeast Alaska. Syesis 17: 95-100.

Nelson, Margaret A.

1992 Shell Midden Deposits and the Archaeobotanical Record. In Deciphering a Shell Midden, edited by Julie K. Stein, pp. 239-256. Academic Press, San Diego. 
Oonk, S., C.P. Slomp, D.J. Huisman, and S.P. Vriend

2009 Effects of site lithology on geochemical signatures of human occupation in archaeological house plans in the Netherlands. Journal of Archaeological Science 36(6): 1215-1228.

Oonk, Stijn, and Job Spijker

2015 A supervised machine-learning approach towards geochemical predictive modelling in archaeology. Journal of Archaeological Science 59: 80-88.

Parnell, J. Jacob, Richard E. Terry, and Charles Golden

2001 Using in-field phosphate testing to rapidly identify middens at Piedras Negras, Guatemala. Geoarchaeology 16(8): 855-873.

Parnell, J. Jacob, Richard E Terry, and Payson Sheets

2002 Soil Chemical Analysis of Ancient Activities in Ceren, El Salvador: A Case Study of a Rapidly Abandoned Site. Latin American Antiquity 13(3): 331-342.

Parnell, Jacob, Richard E. Terry, and Zachary Nelson

2002 Soil Chemical Analysis Applied as an Interpretive Tool for Ancient Human Activities in Piedras Negras, Guatemala. Journal of Archaeological Science 29: 379-404.

Parsons, Jeffrey R.

1972 Archaeological Settlement Patterns. Annual Review of Anthropology 1(1): 127-150.

Percy, R.

1974 The Prehistoric Culture Sequence at Crescent Beach, British Columbia. M.A. Thesis, Department of Archaeology, Simon Fraser University.

Provan, D.M.J.

1971 Soil Phosphate Analysis as a Tool in Archaeology. Norwegian Archaeological Review 4: 37-50.

Sanchez, A., M.L. Canabate, and R. Lizcano

1996 Phosphorus Analysis at Archaeological Sites: An Optimization of the Method and Interpretation of the Results. Archaeometry 38(1): 151-164. 
Schlezinger, David R., and Brian L. Howes

2000 Organic Phosphorus and Elemental Ratios as Indicators of Prehistoric Human Occupation. Journal of Archaeological Science 27(6): 479-492.

Stein, Julie K.

1986 Coring Archaeological Sites. American Antiquity 51(3): 505-527.

1987 Deposits for Archaeologists. Advances in Archaeological Method and Theory 11: 337395.

1992a Deciphering a Shell Midden. Academic Press.

1992b Sediment Analysis of the British Camp Shell Midden. In Deciphering a Shell Midden, edited by Julie K. Stein, pp. 135-160. Academic Press, San Diego.

2008 Geoarchaeology and Archaeostratigraphy: View from a Northwest Coast Shell Midden. In Case Studies in Environmental Archaeology, edited by Elizabeth J. Reitz, Margaret C. Scarry, and Sylvia J. Scudder, pp. 61-80. Springer.

Stein, Julie K., Jennie N. Deo, and Laura S. Phillips

2003 Big Sites - Short Time: Accumulation Rates in Archaeological Sites. Journal of Archaeological Science 30(3): 297-316.

Stein, Julie K., Kimberly D. Kornbacher, and Jason L. Tyler

1992 British Camp Shell Midden Stratigraphy. In Deciphering a Shell Midden, edited by Julie K. Stein, pp. 95-132. Academic Press, San Diego.

Sullivan, K.A., and L. Kealhofer

2004 Identifying activity areas in archaeological soils from a colonial Virginia house lot using phytolith analysis and soil chemistry. Journal of Archaeological Science 31(12): 16591673.

Terry, Richard E., Perry J. Hardin, Stephen D. Houston, Sheldon D. Nelson, Mark W. Jackson, and Jared Carr

2000 Quantitative Phosphorus Measurement: A Field Test Procedure for Archaeological Site Analysis at Piedras Negras, Guatemala. Geoarchaeology: An International Journal 15(2): 151-166. 
Tobiasz, Mary Lynn

2015 Prehistoric Settlement Patterns on the Central Coast of British Columbia. M.A. Thesis, Department of Anthropology, McMaster University.

Trace, Andrew Alexander

1981 An Examination of the Locarno Beach Phase as Represented at the Crescent Beach Site, DgRr 1, British Columbia. M.A. Thesis, Department of Archaeology, Simon Fraser University.

Trant, Andrew J., Wiebe Nijland, Kira M. Hoffman, Darcy L. Mathews, Duncan McLaren, Trisalyn A. Nelson, and Brian M. Starzomski

2016 Intertidal resource use over millennia enhances forest productivity. Nature Communications 7: 1-8.

Trigger, Bruce G.

1967 Settlement Archaeology. Its Goals and Promise. American Antiquity 32(2): 149-160.

Vyncke, K., P. Degryse, E. Vassilieva, and M. Waelkens

2011 Identifying domestic functional areas. Chemical analysis of floor sediments at the Classical-Hellenistic settlement at Düzen Tepe (SW Turkey). Journal of Archaeological Science 38(9): 2274-2292.

Waselkov, Gregory A.

1987 Shellfish Gathering and Shell Midden Archaeology. Advances in Archaeological Method and Theory 10: 93-210.

Whittaker, Fran H., and Julie K. Stein

1992 Shell Midden Boundaries in Relation to Past and Present Shorelines. In Deciphering a Shell Midden, edited by Julie K. Stein, pp. 25-40. Academic Press, San Diego.

Willey, G.R.

1953 Prehistoric Settlement Patterns in the Viru Valley, Peru. Bureau of American Ethnology Bulletin 155: 453.

1956 Prehistoric Settlement Patterns in the New World. Wenner-Gren Foundation, New York. 


\section{Appendix A. Prince Rupert Harbour Analysis}

As discussed in Chapter 4, I had the opportunity to conduct phosphate analysis on sediments from Prince Rupert Harbour (PRH) using the colorimeter. Brewster and Martindale (2011) conducted a program of core- and auger-sampling at a number of sites on the Dundas Islands just west of Prince Rupert following Cannon's (2000a, 2000b) methods. They analyzed fish bones in the same manner and found that fish bone densities (NISP/L) were much lower than those observed on the central coast (Brewster and Martindale 2011:259). Similar low values have been recorded for sites within PRH recently tested by Martindale (Cannon pers. comm.). Major villages and other residential sites in PRH that would be expected to exhibit high densities on the central coast instead had densities comparable to small camps.

Brewster and Martindale (2011:259) conclude that fewer fish remains were deposited in PRH sites compared to other places on the coast. But presumably, PRH sites are similar to central coast sites in the relative intensity of their residential activities. As an independent measure of occupational intensity, it follows that phosphate values from PRH should fall within the same range of values evident for the central coast, regardless of fish bone densities.

To test this, two sites were chosen from PRH. One site (GbTo-4) is a larger site, which is considered a major village, and which exhibited the highest FBD of the PRH sites recently sampled by Martindale; the other (GbTo-1) is a smaller site that exhibited a relatively low FBD value. If GbTo-4 is similar in its residential intensity to village sites on the central coast, it should have a relatively high phosphate value. If GbTo-1 falls within the range for the central coast, it would have a relatively lower phosphate value. Table A1 shows the results of these tests.

Table A1. Results of PRH phosphate analysis

\begin{tabular}{c|rr} 
Site & Sample depth $(\mathrm{cm})$ & Phosphate $(\mathrm{ppb})$ \\
\hline GbTo-4 & $30-45$ & 5676 \\
& $150-175$ & 4322 \\
& $250-265$ & 6034 \\
& AVERAGE & 5344 \\
GcTo-1 & AVERAGE ROUNDED & $\mathbf{5 3 0 0}$ \\
\cline { 2 - 3 } & $60-75$ & 2219 \\
& $165-180$ & 3604 \\
& $255-270$ & 716 \\
& AVERAGE & 2180 \\
& AVERAGE ROUNDED & $\mathbf{2 2 0 0}$ \\
\hline
\end{tabular}




\section{M.A. Thesis - K. Carter; McMaster University - Anthropology}

As expected GbTo-4 has a higher phosphate value than GcTo-1. Site GbTo-4 also falls within the range expected for central coast villages, ranking between McNaughton (ElTb-10) and Namu (ElSx-1). Site GcTo-1 falls within the range expected for central coast camp sites, ranking between ElSx-8 and ElSx-5, which are a camp and a base camp, respectively.

These results suggest that the intensity of residential activity among PRH sites is distributed in a manner similar to what has been observed on the central coast, and that phosphate analysis can address questions of occupational intensity in $\mathrm{PRH}$, and presumably elsewhere on the Northwest Coast, though it cannot speak to the broader question of differences in fish bone density between the central coast and PRH.

\section{References Cited (Appendix A)}

Brewster, Natalie, and Andrew Martindale

2011 An Archaeological History of Holocene Fish Use in the Dundas Island Group, British Columbia. In The Archaeology of North Pacific Fisheries, edited by Madonna L. Moss and Aubrey Cannon, pp. 247-264. University of Alaska Press, Fairbanks.

Cannon, Aubrey

2000a Assessing Variability in Northwest Coast Salmon and Herring Fisheries: Bucket-Auger Sampling of Shell Midden Sites on the Central Coast of British Columbia. Journal of Archaeological Science 27(8): 725-737.

2000b Settlement and Sea-Levels on the Central Coast of British Columbia: Evidence from Shell Midden Cores. American Antiquity 65(1): 67-77. 


\section{Appendix B. LaMotte SMART3 Colorimeters put to the test}

I had the opportunity to run samples on a second colorimeter located at Sustainable Archaeology McMaster (SAM). My goal was simply to see if this colorimeter would reproduce the same values I had obtained using the one located in the Fisheries Archaeology Lab (FAL). Because I had recently run the PRH samples at the FAL, I chose these to run again at SAM. Results came out as expected. Both colorimeters produced very comparable - if not identical site-level values (see Table B1). In addition, results from site GbTo-4 are over range (see Chapter 3 for a discussion of this). As mentioned in Chapter 3, I did not correct for over range values. Instead, I used the estimated value generated by the colorimeter. Despite some inconsistencies in the values reported for GbTo-4, all samples still fall in the same rank order based on the results from either colorimeter. These results further indicate that this application is most useful for site-level, rank order comparisons and that the results generated for this thesis can be replicated using another colorimeter.

TABLE B1. Comparison of PRH results using two different colorimeters.

\begin{tabular}{|rrr|rrr|}
\hline \multicolumn{2}{|c}{ GbTo-4 } & \multicolumn{3}{c|}{ GcTo-1 } \\
Sample Depth $(\mathrm{cm})$ & \multicolumn{2}{c}{ Phosphate $(\mathrm{ppb})$} & & \multicolumn{2}{c|}{ Phosphate $(\mathrm{ppb})$} \\
\cline { 2 - 6 } \cline { 5 - 6 } & FAL & SAM & Sample Depth $(\mathrm{cm})$ & FAL & SAM \\
\hline $30-45$ & 5676 & 5649 & $60-75$ & 2219 & 2434 \\
$150-175$ & 4322 & 4225 & $165-180$ & 3604 & 3536 \\
$250-265$ & 6034 & 5659 & $255-270$ & 716 & 729 \\
AVERAGE & 5344 & 5178 & AVERAGE & 2180 & 2233 \\
AVERAGE ROUNDED & $\mathbf{5 3 0 0}$ & $\mathbf{5 2 0 0}$ & AVERAGE ROUNDED & $\mathbf{2 2 0 0}$ & $\mathbf{2 2 0 0}$ \\
\hline
\end{tabular}




\section{Appendix C. Major Shell Midden Constituents: How much phosphate?}

Shell middens are composed largely of shell, ash, charcoal and fire-cracked rock, among other constituents. As a rough way to assess potentially how much available phosphate some of these individual constituents contribute to shell midden matrix, I tested wood ash from a fireplace, charcoal from Namu, and crushed clam and mussel shell using the colorimeter. I also tested a $100 \%$ dried salmon dog treat. By testing all of these potential phosphate contributors, I aimed to get an idea of which, if any, constituents contributed more phosphate than others. Of course, this experiment is only a very rough indication of a few potential contributors. That said, my expectation was that the dog treat would be highest in phosphate, being dried meat. Phosphorus is also associated with hearths, so it was possible that charcoal would be high as well. Table D1 shows the results of this experiment.

As expected, the dried salmon exhibited the highest available phosphate value. The charcoal also read very high. As indicated in Table C1, the mussel shell I crushed was taken from a restaurant and contained bits of dried mussel. Although marine shells are different in their chemical composition, the heightened phosphate value of the mussel shell compared to the clam shell may be due to the presence of meat. Also, the dried salmon did read highest, though I hesitate to suggest that the result has much bearing. This phosphate test is designed for use with soils and sediments, not dried meat protein. Unanticipated reactions occurred during both the extraction procedure and subsequent test for phosphate. During the extraction, much of the dried, ground salmon dissolved in the solution, making it rather gelatinous. The filtering took much longer as a result. Once the phosphate acid reagent was added to the reagent blank, the liquid yellowed and coagulated (not unlike the reaction that occurs when one intentionally sours milk for biscuits...). Clearly, this does not happen with soils or sediments. The phosphate reducing reagent did not physically change the solution and the anticipated blue colour did form, indicating the presence of phosphate, but it was concentrated on the curdled protein. Swirling the sample before placing it into the colorimeter for reading was sufficient to break up the coagulation, but the solution remained clouded. I cannot say that the result from this test is comparable to any others. 


\section{M.A. Thesis - K. Carter; McMaster University - Anthropology}

Table C1. Results of experimental analysis of individual midden 'constituents'.

\begin{tabular}{lrrl}
\multicolumn{1}{c}{ Constituent } & $\begin{array}{c}\text { Phosphate } \\
(\mathrm{ppb})\end{array}$ & $\begin{array}{r}\text { Phosphate } \\
(\mathrm{ppb}) \text { RND }\end{array}$ & Notes \\
\hline wood ash & 1717 & $\mathbf{1 7 0 0}$ & From a modern fire place \\
crushed clam shell & 111 & $\mathbf{1 0 0}$ & Archaeological clam shell from exposure \\
crushed mussel shell & 942 & $\mathbf{9 0 0}$ & $\begin{array}{l}\text { Modern mussel shell from restaurant; not cleaned } \\
\text { (small bits of meat still attached when ground) }\end{array}$ \\
charcoal & 11390 & $\mathbf{1 1 4 0 0}$ & $\begin{array}{l}\text { Pure archaeological charcoal; floated. Likely floated } \\
\text { uither } \mathrm{CCl}_{4} \text { or } \mathrm{ZnCl}_{2} \text {. May also have been floated }\end{array}$ \\
dried salmon & 12421 & $\mathbf{1 2 4 0 0}$ & Dog treat made of '100\% salmon'
\end{tabular}

The charcoal sample tested here was from column samples collected at Namu in 1970 and floated in 1976. Although I do not know how the sample was floated, popular chemicals used for floatation at the time include carbon tetrachloride $\left(\mathrm{CCl}_{4}\right)$ and zinc chloride $\left(\mathrm{ZnCl}_{2}\right)$; salt water $(\mathrm{NaCl})$ can also be used (Watson 1976). Based on this quick review it does not seem that any chemicals used for floatation would add phosphate to the chemical signature, but I cannot be certain.

At the very least, this experiment shows that not all contributors to shell midden matrix are equal. Some contribute more phosphate than others. Phosphate was present in all the samples tested, indicating further that it is a measure of multiple activities simultaneously. Interestingly, charcoal contains very high amounts of phosphate, even compared to wood ash (though, see disclaimer above). Perhaps charcoal is the main contributor to the phosphate signature in hearth areas. For the purposes of this research, fish and charcoal seem to be large contributors of available phosphate, which is consistent with the results presented in this thesis - high phosphate values were observed at fish processing sites and large villages, which probably concentrated more fish and charcoal relative to other locations.

\section{References Cited (Appendix C)}

Watson, Patty Jo

1976 In Pursuit of Prehistoric Subsistence: A Comparative Account of some Contemporary Floatation Techniques. Midcontinental Journal of Archaeology 1(1): 77-100. 
M.A. Thesis - K. Carter; McMaster University - Anthropology

\section{Appendix D: Raw Data}

\begin{tabular}{|c|c|c|c|c|c|c|c|}
\hline Site & Site Name/Location & $\begin{array}{c}\text { Site Area } \\
\left(\mathrm{m}^{2}\right)\end{array}$ & Auger & $\begin{array}{l}\text { Depth }^{1} \\
\text { (cm bs) }\end{array}$ & $\begin{array}{l}\text { Phosphate } \\
\text { (ppb) }\end{array}$ & $\begin{array}{l}\text { Fish Bones } \\
\text { (NISP) }\end{array}$ & $\begin{array}{c}\text { Vol. }<2 \mathrm{~mm} \\
(\mathrm{~mL})\end{array}$ \\
\hline EISx-1 & Namu & 8100 & $\mathrm{~A} 1$ & 74 & 6840 & $70^{*}$ & $225^{*}$ \\
\hline EISX-1 & Namu & 8100 & $\mathrm{~A} 2$ & 80 & 4728 & $6^{*}$ & $170^{*}$ \\
\hline EISx-1 & Namu & 8100 & $A 3$ & 99 & 4634 & $67^{*}$ & $175^{*}$ \\
\hline EISX-1 & Namu & 8100 & A4 & 109 & 4810 & $12^{*}$ & $150^{*}$ \\
\hline EISx-1 & Namu & 8100 & A5 & 125 & 4647 & $9^{*}$ & $200^{*}$ \\
\hline EISx-1 & Namu & 8100 & A6 & 137 & 4816 & $15^{*}$ & $190^{*}$ \\
\hline EISx-1 & Namu & 8100 & A7 & 143 & 4498 & $40^{*}$ & $235^{*}$ \\
\hline EISx-1 & Namu & 8100 & A8 & 150 & 5174 & $23^{*}$ & $225^{*}$ \\
\hline ElSx-1 & Namu & 8100 & A9 & 162 & 5736 & $34^{*}$ & $225^{*}$ \\
\hline EISx-1 & Namu & 8100 & A10 & 171 & 5816 & $49^{*}$ & $250^{*}$ \\
\hline EISx-1 & Namu & 8100 & A11 & 183 & 5363 & $26^{*}$ & $350^{*}$ \\
\hline EISx-1 & Namu & 8100 & A12 & 196 & 5835 & $34^{*}$ & $190^{*}$ \\
\hline EISX-1 & Namu & 8100 & A13 & 210 & 4640 & $47^{*}$ & $195^{*}$ \\
\hline EISX-1 & Namu & 8100 & A14 & 221 & 5071 & $84^{*}$ & $265^{*}$ \\
\hline EISX-1 & Namu & 8100 & A15 & 236 & 4420 & $25^{*}$ & $220^{*}$ \\
\hline EISX-1 & Namu & 8100 & A16 & 244 & 5073 & $8^{*}$ & $235^{*}$ \\
\hline EISx-1 & Namu & 8100 & A17 & 254 & 6321 & $10^{*}$ & $230^{*}$ \\
\hline EISx-1 & Namu & 8100 & A18 & 267 & 5565 & $4^{*}$ & $225^{*}$ \\
\hline EISx-1 & Namu & 8100 & A19 & 284 & 4722 & $13^{*}$ & $185^{*}$ \\
\hline EISx-1 & Namu & 8100 & $\mathrm{~A} 20$ & 308 & 7383 & $17^{*}$ & $170^{*}$ \\
\hline EISx-1 & Namu & 8100 & $\mathrm{~A} 21$ & 311 & 5084 & $66^{*}$ & $245^{*}$ \\
\hline EISX-1 & Namu & 8100 & A22 & 328 & 8871 & $54^{*}$ & $250^{*}$ \\
\hline EISX-1 & Namu & 8100 & A23 & 343 & 8711 & $11^{*}$ & $315^{*}$ \\
\hline EISX-1 & Namu & 8100 & A24 & 359 & 3680 & $110^{*}$ & $385^{*}$ \\
\hline ElSx-1 & Namu & 8100 & A25 & 357 & 3851 & $33^{*}$ & $260^{*}$ \\
\hline EISX-1 & Namu & 8100 & A26 & 361 & 4425 & $5^{*}$ & $210^{*}$ \\
\hline EISX-1 & Namu & 8100 & AVERAGE & --- & 5412 & --- & --- \\
\hline ElSx-1 & Namu & 8100 & FS10.1 & ?? & 5547 & ?? & ?? \\
\hline ElSx-1 & Namu & 8100 & FS10.2 & ?? & 3013 & ?? & ?? \\
\hline EISx-1 & Namu & 8100 & FS10.5 & ?? & 2701 & ?? & ?? \\
\hline EISx-1 & Namu & 8100 & FS10.6 & ?? & 6785 & ?? & ?? \\
\hline EISx-1 & Namu & 8100 & FS10.7 & ?? & 12259 & ?? & ?? \\
\hline EISx-1 & Namu & 8100 & FS10.8 & ?? & 6249 & ?? & ?? \\
\hline EISx-1 & Namu & 8100 & FS10.9 & ?? & 5974 & ?? & ?? \\
\hline ElSx-1 & Namu & 8100 & FS10.10 & ?? & 6299 & ?? & ?? \\
\hline ElSx-1 & Namu & 8100 & FS10.11 & ?? & 10408 & ?? & ?? \\
\hline EISX-1 & Namu & 8100 & FS10.12 & ?? & 6758 & ?? & $? ?$ \\
\hline ElSx-1 & Namu & 8100 & FS10.13 & ?? & 8976 & ?? & ?? \\
\hline ElSx-1 & Namu & 8100 & FS10.15 & ?? & 4347 & ?? & ?? \\
\hline EISX-1 & Namu & 8100 & FS10.16 & ?? & 6448 & ?? & ?? \\
\hline EISx-1 & Namu & 8100 & FS10.17 & ?? & 4244 & ?? & ?? \\
\hline EISx-1 & Namu & 8100 & AVERAGE & --- & 6429 & --- & --- \\
\hline
\end{tabular}


M.A. Thesis - K. Carter; McMaster University - Anthropology

\begin{tabular}{|c|c|c|c|c|c|c|c|}
\hline Site & Site Name/Location & $\begin{array}{c}\text { Site Area } \\
\left(\mathrm{m}^{2}\right)\end{array}$ & Auger & $\begin{array}{l}\text { Depth }^{1} \\
\text { (cm bs) }\end{array}$ & $\begin{array}{c}\text { Phosphate } \\
\text { (ppb) }\end{array}$ & $\begin{array}{c}\text { Fish Bones } \\
\text { (NISP) }\end{array}$ & $\begin{array}{c}\text { Vol. }<2 \mathrm{~mm} \\
\quad(\mathrm{~mL})\end{array}$ \\
\hline EISx-3 & Kisameet & 1630 & $\mathrm{C} 1$ & 17 & 3826 & 3 & 150 \\
\hline EISx-3 & Kisameet & 1630 & $\mathrm{C} 2$ & 39 & 4088 & 73 & 200 \\
\hline EISx-3 & Kisameet & 1630 & $\mathrm{C} 3$ & 63 & 2095 & 46 & 310 \\
\hline EISx-3 & Kisameet & 1630 & $\mathrm{C} 4$ & 81 & 4781 & 55 & 250 \\
\hline EISx-3 & Kisameet & 1630 & C5 & 96 & 4919 & 53 & 235 \\
\hline EISx-3 & Kisameet & 1630 & C6 & 105 & 4467 & 26 & 215 \\
\hline EISX-3 & Kisameet & 1630 & $\mathrm{C7}$ & 120 & 5483 & 15 & 165 \\
\hline EISx-3 & Kisameet & 1630 & $\mathrm{C} 8$ & 144 & 7384 & 110 & 250 \\
\hline EIS $x-3$ & Kisameet & 1630 & $\mathrm{C9}$ & 157 & 4703 & 26 & 140 \\
\hline EISx-3 & Kisameet & 1630 & C10 & 176 & 3808 & 7 & 95 \\
\hline EISX-3 & Kisameet & 1630 & C11 & 192 & 3577 & 4 & 125 \\
\hline EISx-3 & Kisameet & 1630 & C12 & 202 & 2720 & 5 & 110 \\
\hline EISx-3 & Kisameet & 1630 & C13 & 213 & 1428 & 1 & 120 \\
\hline EISx-3 & Kisameet & 1630 & C14 & 230 & 2123 & 2 & 160 \\
\hline EISX-3 & Kisameet & 1630 & C15 & 229 & 2621 & 4 & 75 \\
\hline EISx-3 & Kisameet & 1630 & C16 & 242 & 5701 & 97 & 390 \\
\hline EISX-3 & Kisameet & 1630 & C17 & 247 & 4112 & 13 & 85 \\
\hline EISX-3 & Kisameet & 1630 & C18 & 250 & 3660 & 12 & 125 \\
\hline EISX-3 & Kisameet & 1630 & C19 & 256 & 3988 & 12 & 150 \\
\hline EISX-3 & Kisameet & 1630 & C2O & 259 & 3925 & 14 & 200 \\
\hline EISx-3 & Kisameet & 1630 & C21 & 272 & 3213 & 6 & 270 \\
\hline EISX-3 & Kisameet & 1630 & AVERAGE & --- & 3934 & --- & --- \\
\hline EISx-4 & Windsor Cove & 140 & A1 & 54 & 1614 & 0 & 215 \\
\hline EISx-4 & Windsor Cove & 140 & $\mathrm{~A} 2$ & 71 & 704 & 2 & 165 \\
\hline EISx-4 & Windsor Cove & 140 & A3 & 92 & 732 & 10 & 430 \\
\hline EISx-4 & Windsor Cove & 140 & A4 & 106 & 724 & 6 & 240 \\
\hline EISx-4 & Windsor Cove & 140 & A5 & 133 & 250 & 4 & 300 \\
\hline EISx-4 & Windsor Cove & 140 & A6 & 152 & 177 & 1 & 390 \\
\hline EISx-4 & Windsor Cove & 140 & A7 & 161 & 112 & 2 & 240 \\
\hline EISx-4 & Windsor Cove & 140 & AVERAGE & --- & 616 & --- & --- \\
\hline EISx-5 & Windsor Cove & 2190 & A1 & 53 & 2531 & 8 & 350 \\
\hline EISX-5 & Windsor Cove & 2190 & $\mathrm{~A} 2$ & 69 & 1102 & 52 & 340 \\
\hline EISx-5 & Windsor Cove & 2190 & A3 & 75 & 1837 & 11 & 285 \\
\hline EISx-5 & Windsor Cove & 2190 & A4 & 76 & 2047 & 29 & 355 \\
\hline EISx-5 & Windsor Cove & 2190 & A5 & 81 & 3705 & 5 & 390 \\
\hline EISx-5 & Windsor Cove & 2190 & A6 & 94 & 3258 & 18 & 345 \\
\hline EISx-5 & Windsor Cove & 2190 & A7 & 111 & 4337 & 18 & 285 \\
\hline EISx-5 & Windsor Cove & 2190 & A8 & 120 & 3396 & 6 & 200 \\
\hline EISx-5 & Windsor Cove & 2190 & A9 & 131 & 1637 & 6 & 225 \\
\hline EISx-5 & Windsor Cove & 2190 & A10 & 147 & 1087 & 2 & 275 \\
\hline EISx-5 & Windsor Cove & 2190 & A11 & 163 & 1033 & 13 & 270 \\
\hline EISx-5 & Windsor Cove & 2190 & A12 & 189 & 3676 & 81 & 530 \\
\hline EIS $x-5$ & Windsor Cove & 2190 & A13 & 202 & 2576 & 53 & 480 \\
\hline EISx-5 & Windsor Cove & 2190 & A14 & 222 & 2891 & 39 & 440 \\
\hline
\end{tabular}


M.A. Thesis - K. Carter; McMaster University - Anthropology

\begin{tabular}{|c|c|c|c|c|c|c|c|}
\hline Site & Site Name/Location & $\begin{array}{c}\text { Site Area } \\
\left(\mathrm{m}^{2}\right)\end{array}$ & Auger & $\begin{array}{l}\text { Depth }^{1} \\
\text { (cm bs) }\end{array}$ & $\begin{array}{c}\text { Phosphate } \\
\text { (ppb) }\end{array}$ & $\begin{array}{c}\text { Fish Bones } \\
\text { (NISP) }\end{array}$ & $\begin{array}{c}\text { Vol. }<2 \mathrm{~mm} \\
(\mathrm{~mL})\end{array}$ \\
\hline EISx-5 & Windsor Cove & 2190 & A15 & 242 & 6368 & 58 & 340 \\
\hline EISx-5 & Windsor Cove & 2190 & A16 & 258 & 3985 & 52 & 350 \\
\hline EISx-5 & Windsor Cove & 2190 & A17 & 268 & 3874 & 19 & 275 \\
\hline EISx-5 & Windsor Cove & 2190 & A18 & 289 & 3302 & 15 & 200 \\
\hline EISx-5 & Windsor Cove & 2190 & A19 & 306 & 2511 & 29 & 440 \\
\hline EISx-5 & Windsor Cove & 2190 & A20 & 320 & 2690 & 20 & 700 \\
\hline EISx-5 & Windsor Cove & 2190 & A21 & 341 & 1340 & 14 & 365 \\
\hline EISX-5 & Windsor Cove & 2190 & AVERAGE & --- & 2818 &.-- & $-\cdots$ \\
\hline EISx-8 & Fougner Bay & 930 & A1 & 46 & 3410 & 0 & 165 \\
\hline EISx-8 & Fougner Bay & 930 & $\mathrm{~A} 2$ & 65 & 4545 & 0 & 200 \\
\hline EISx-8 & Fougner Bay & 930 & A3 & 78 & 355 & 1 & 200 \\
\hline EISx-8 & Fougner Bay & 930 & A4 & 87 & 508 & 0 & 130 \\
\hline EISx-8 & Fougner Bay & 930 & A5 & 99 & 525 & 3 & 115 \\
\hline EISx-8 & Fougner Bay & 930 & A6 & 112 & 1267 & 3 & 265 \\
\hline EISx-8 & Fougner Bay & 930 & A7 & 122 & 1358 & 0 & 200 \\
\hline EISx-8 & Fougner Bay & 930 & A8 & 134 & 1421 & 0 & 125 \\
\hline EISx-8 & Fougner Bay & 930 & A9 & 143 & 759 & 0 & 125 \\
\hline EISx-8 & Fougner Bay & 930 & A10 & 148 & 478 & 0 & 25 \\
\hline EISx-8 & Fougner Bay & 930 & AVERAGE & --- & 1463 & --- & --- \\
\hline EISx-10 & Fougner Bay & 840 & D1 & 55 & 6181 & 16 & 325 \\
\hline EISx-10 & Fougner Bay & 840 & D2 & 72 & 4452 & 24 & 220 \\
\hline EISx-10 & Fougner Bay & 840 & D3 & 84 & 4191 & 9 & 230 \\
\hline EISx-10 & Fougner Bay & 840 & D4 & 102 & 3731 & 14 & 225 \\
\hline EISx-10 & Fougner Bay & 840 & D5 & 121 & 4553 & 43 & 300 \\
\hline EISx-10 & Fougner Bay & 840 & D6 & 130 & 3668 & 15 & 215 \\
\hline EISx-10 & Fougner Bay & 840 & D7 & 139 & 3636 & 17 & 215 \\
\hline EISx-10 & Fougner Bay & 840 & D8 & 149 & 4161 & 1 & 175 \\
\hline EIS $x-10$ & Fougner Bay & 840 & D9 & 161 & 3438 & 7 & 200 \\
\hline EISx-10 & Fougner Bay & 840 & D10 & 178 & 2502 & 5 & 190 \\
\hline EISx-10 & Fougner Bay & 840 & D11 & 198 & 3332 & 8 & 225 \\
\hline EISx-10 & Fougner Bay & 840 & D12 & 223 & 2993 & 19 & 220 \\
\hline EISx-10 & Fougner Bay & 840 & D13 & 238 & 2940 & 2 & 140 \\
\hline EISx-10 & Fougner Bay & 840 & D14 & 252 & 3455 & 28 & 235 \\
\hline EISX-10 & Fougner Bay & 840 & AVERAGE & --- & 3802 & $-\cdots$ & --- \\
\hline EISx-16 & Fougner Bay & 300 & A1 & 33 & 3624 & 1 & 175 \\
\hline EISx-16 & Fougner Bay & 300 & $\mathrm{~A} 2$ & 52 & 1611 & 52 & 135 \\
\hline EISx-16 & Fougner Bay & 300 & A3 & 68 & 1111 & 11 & 100 \\
\hline EISx-16 & Fougner Bay & 300 & A4 & 85 & 1061 & 6 & 190 \\
\hline EISx-16 & Fougner Bay & 300 & A5 & 114 & 2649 & 5 & 175 \\
\hline EISx-16 & Fougner Bay & 300 & A6 & 120 & 1093 & 20 & 200 \\
\hline EISx-16 & Fougner Bay & 300 & A7 & 129 & 290 & 9 & 145 \\
\hline EISx-16 & Fougner Bay & 300 & A8 & 142 & 343 & 3 & 100 \\
\hline EISx-16 & Fougner Bay & 300 & A9 & 168 & 666 & 6 & 300 \\
\hline EISx-16 & Fougner Bay & 300 & AVERAGE & --- & 1383 & --- & --- \\
\hline
\end{tabular}


M.A. Thesis - K. Carter; McMaster University - Anthropology

\begin{tabular}{|c|c|c|c|c|c|c|c|}
\hline Site & Site Name/Location & $\begin{array}{c}\text { Site Area } \\
\left(\mathrm{m}^{2}\right)\end{array}$ & Auger & $\begin{array}{l}\text { Depth }^{1} \\
\text { (cm bs) }\end{array}$ & $\begin{array}{c}\text { Phosphate } \\
\text { (ppb) }\end{array}$ & $\begin{array}{c}\text { Fish Bones } \\
\text { (NISP) }\end{array}$ & $\begin{array}{c}\text { Vol. }<2 \mathrm{~mm} \\
\quad(\mathrm{~mL})\end{array}$ \\
\hline EISX-18 & Fougner Bay & 1310 & A1 & 42 & 2115 & 1 & 70 \\
\hline EISx-18 & Fougner Bay & 1310 & A3 & 80 & 5143 & 16 & 300 \\
\hline EISX-18 & Fougner Bay & 1310 & A5 & 108 & 5381 & 32 & 310 \\
\hline EISx-18 & Fougner Bay & 1310 & A7 & 135 & 4228 & 32 & 170 \\
\hline EISx-18 & Fougner Bay & 1310 & A9 & 173 & 3041 & 6 & 150 \\
\hline EISX-18 & Fougner Bay & 1310 & A11 & 205 & 3133 & 1 & 125 \\
\hline EISX-18 & Fougner Bay & 1310 & A13 & 236 & 3446 & 16 & 120 \\
\hline EISx-18 & Fougner Bay & 1310 & A15 & 264 & 3574 & 43 & 95 \\
\hline EISX-18 & Fougner Bay & 1310 & AVERAGE & --- & 3758 & --- & --- \\
\hline EITa-3 & Watt Bay & 50 & A1 & 45 & 4032 & 1 & 225 \\
\hline EITa-3 & Watt Bay & 50 & $\mathrm{~A} 2$ & 58 & 2198 & 7 & 220 \\
\hline EITa-3 & Watt Bay & 50 & A3 & 70 & 4109 & 9 & 210 \\
\hline EITa-3 & Watt Bay & 50 & A4 & 84 & 4162 & 20 & 315 \\
\hline EITa-3 & Watt Bay & 50 & A5 & 95 & 8251 & 29 & 240 \\
\hline EITa-3 & Watt Bay & 50 & A6 & 102 & 4801 & 1 & 250 \\
\hline EITa-3 & Watt Bay & 50 & A7 & 115 & 10212 & 39 & 260 \\
\hline EITa-3 & Watt Bay & 50 & A8 & 128 & 12202 & 31 & 245 \\
\hline ElTa-3 & Watt Bay & 50 & AVERAGE & $-\cdots$ & 6246 & $-\cdots$ & --- \\
\hline EITa-18 & Kildidt Inlet & 1310 & B1 & 52 & 6070 & 0 & 445 \\
\hline EITa-18 & Kildidt Inlet & 1310 & B3 & 91 & 3717 & 0 & 635 \\
\hline EITa-18 & Kildidt Inlet & 1310 & B5 & 119 & 4554 & 0 & 475 \\
\hline EITa-18 & Kildidt Inlet & 1310 & B7 & 143 & 4567 & 0 & 375 \\
\hline EITa-18 & Kildidt Inlet & 1310 & B9 & 169 & 5867 & 2 & 215 \\
\hline EITa-18 & Kildidt Inlet & 1310 & B11 & 204 & 5334 & 0 & 640 \\
\hline ElTa-18 & Kildidt Inlet & 1310 & AVERAGE & --- & 5018 & -- & -- \\
\hline EITa-21 & Kildidt Lagoon & 130 & A1 & 24 & 5017 & 0 & 310 \\
\hline EITa-21 & Kildidt Lagoon & 130 & $\mathrm{~A} 2$ & 38 & 8081 & 0 & 315 \\
\hline EITa-21 & Kildidt Lagoon & 130 & A3 & 53 & 12027 & 6 & 350 \\
\hline EITa-21 & Kildidt Lagoon & 130 & A4 & 69 & 12532 & 6 & 395 \\
\hline EITa-21 & Kildidt Lagoon & 130 & A5 & 81 & 8843 & 0 & 430 \\
\hline EITa-21 & Kildidt Lagoon & 130 & A6 & 82 & 11405 & 1 & 290 \\
\hline EITa-21 & Kildidt Lagoon & 130 & AVERAGE & --- & 9651 & $-\cdots$ & --- \\
\hline ElTb-1 & Nulu & 1110 & A1 & 51 & 4475 & 8 & 355 \\
\hline ElTb-1 & Nulu & 1110 & $\mathrm{~A} 2$ & 64 & 3974 & 30 & 270 \\
\hline ElTb-1 & Nulu & 1110 & A3 & 76 & 4360 & 6 & 225 \\
\hline ElTb-1 & Nulu & 1110 & A4 & 87 & 5227 & 58 & 205 \\
\hline ElTb-1 & Nulu & 1110 & A5 & 110 & 4163 & 21 & 150 \\
\hline ElTb-1 & Nulu & 1110 & A6 & 118 & 3958 & 35 & 210 \\
\hline ElTb-1 & Nulu & 1110 & A7 & 129 & 3249 & 25 & 145 \\
\hline ElTb-1 & Nulu & 1110 & A8 & 140 & 3457 & 22 & 275 \\
\hline ElTb-1 & Nulu & 1110 & A9 & 146 & 4990 & 30 & 300 \\
\hline ElTb-1 & Nulu & 1110 & A10 & 164 & 3691 & 89 & 640 \\
\hline ElTb-1 & Nulu & 1110 & A11 & 172 & 4463 & 45 & 280 \\
\hline ElTb-1 & Nulu & 1110 & A12 & 174 & 3010 & 64 & 225 \\
\hline
\end{tabular}


M.A. Thesis - K. Carter; McMaster University - Anthropology

\begin{tabular}{|c|c|c|c|c|c|c|c|}
\hline Site & Site Name/Location & $\begin{array}{l}\text { Site Area } \\
\left(\mathrm{m}^{2}\right)\end{array}$ & Auger & $\begin{array}{l}\text { Depth }^{1} \\
\text { (cm bs) }\end{array}$ & $\begin{array}{c}\text { Phosphate } \\
\text { (ppb) }\end{array}$ & $\begin{array}{l}\text { Fish Bones } \\
\text { (NISP) }\end{array}$ & $\begin{array}{c}\text { Vol. }<2 \mathrm{~mm} \\
(\mathrm{~mL})\end{array}$ \\
\hline ElTb-1 & Nulu & 1110 & A13 & 192 & 1827 & 46 & 185 \\
\hline ElTb-1 & Nulu & 1110 & A14 & 213 & 4530 & 267 & 620 \\
\hline ElTb-1 & Nulu & 1110 & A15 & 224 & 1204 & 38 & 410 \\
\hline ElTb-1 & Nulu & 1110 & A16 & 235 & 2953 & 121 & 340 \\
\hline ElTb-1 & Nulu & 1110 & A17 & 247 & 3231 & 49 & 300 \\
\hline ElTb-1 & Nulu & 1110 & A18 & 254 & 4072 & 47 & 335 \\
\hline ElTb-1 & Nulu & 1110 & A19 & 264 & 4442 & 89 & 390 \\
\hline ElTb-1 & Nulu & 1110 & $\mathrm{~A} 20$ & 274 & 5724 & 57 & 325 \\
\hline ElTb-1 & Nulu & 1110 & A21 & 286 & 4002 & 50 & 380 \\
\hline ElTb-1 & Nulu & 1110 & $\mathrm{~A} 22$ & 308 & 4673 & 62 & 420 \\
\hline ElTb-1 & Nulu & 1110 & A23 & 321 & 1505 & 70 & 380 \\
\hline ElTb-1 & Nulu & 1110 & A24 & 345 & 4492 & 103 & 380 \\
\hline ElTb-1 & Nulu & 1110 & AVERAGE & --- & 3820 & --- & --- \\
\hline ElTb-2 & Spitfire Channel & 60 & $\mathrm{~A} 1$ & 27 & 6755 & 2 & 275 \\
\hline ElTb-2 & Spitfire Channel & 60 & $\mathrm{~A} 2$ & 40 & 12105 & 0 & 210 \\
\hline ElTb-2 & Spitfire Channel & 60 & $\mathrm{~A} 3$ & 58 & 13170 & 1 & 320 \\
\hline ElTb-2 & Spitfire Channel & 60 & A4 & 75 & 11296 & 17 & 300 \\
\hline ElTb-2 & Spitfire Channel & 60 & A5 & 85 & 1846 & 7 & 155 \\
\hline ElTb-2 & Spitfire Channel & 60 & A6 & 96 & 3763 & 57 & 260 \\
\hline ElTb-2 & Spitfire Channel & 60 & A7 & 115 & 2924 & 8 & 465 \\
\hline ElTb-2 & Spitfire Channel & 60 & A8 & 124 & 5750 & 4 & 215 \\
\hline ElTb-2 & Spitfire Channel & 60 & A9 & 130 & 8402 & 1 & 290 \\
\hline ElTb-2 & Spitfire Channel & 60 & AVERAGE & --- & 7335 & --- & --- \\
\hline EITb-10 & McNaughton & 4500 & A1 & 24 & 7028 & 0 & 325 \\
\hline ElTb-10 & McNaughton & 4500 & $\mathrm{~A} 2$ & 34 & 4745 & 3 & 500 \\
\hline EITb-10 & McNaughton & 4500 & $\mathrm{~A} 3$ & 44 & 6951 & 33 & 600 \\
\hline ElTb-10 & McNaughton & 4500 & A4 & 54 & 11568 & 118 & 750 \\
\hline EITb-10 & McNaughton & 4500 & A5 & 70 & 5336 & 35 & 1375 \\
\hline EITb-10 & McNaughton & 4500 & A6 & 80 & 6645 & 10 & 850 \\
\hline EITb-10 & McNaughton & 4500 & A7 & 88 & 9805 & 46 & 750 \\
\hline EITb-10 & McNaughton & 4500 & A8 & 98 & 9157 & 140 & 825 \\
\hline EITb-10 & McNaughton & 4500 & A9 & 110 & 4598 & 112 & 875 \\
\hline EITb-10 & McNaughton & 4500 & A10 & 120 & 3751 & 7 & 675 \\
\hline EITb-10 & McNaughton & 4500 & A11 & 130 & 3669 & 59 & 650 \\
\hline EITb-10 & McNaughton & 4500 & A12 & 138 & 8134 & 528 & 550 \\
\hline EITb-10 & McNaughton & 4500 & A13 & 146 & 4682 & 133 & 500 \\
\hline EITb-10 & McNaughton & 4500 & A14 & 154 & 4557 & 28 & 450 \\
\hline EITb-10 & McNaughton & 4500 & A15 & 168 & 4605 & 54 & 650 \\
\hline EITb-10 & McNaughton & 4500 & A16 & 172 & 4976 & 65 & 575 \\
\hline ElTb-10 & McNaughton & 4500 & A17 & 182 & 6290 & 39 & 575 \\
\hline EITb-10 & McNaughton & 4500 & A18 & 190 & 3931 & 14 & 500 \\
\hline EITb-10 & McNaughton & 4500 & A19 & 206 & 5107 & 23 & 400 \\
\hline EITb-10 & McNaughton & 4500 & A20 & 220 & 4660 & 21 & 400 \\
\hline EITb-10 & McNaughton & 4500 & $\mathrm{~A} 21$ & 230 & 4725 & 49 & 375 \\
\hline
\end{tabular}


M.A. Thesis - K. Carter; McMaster University - Anthropology

\begin{tabular}{|c|c|c|c|c|c|c|c|}
\hline Site & Site Name/Location & $\begin{array}{l}\text { Site Area } \\
\left(\mathrm{m}^{2}\right)\end{array}$ & Auger & $\begin{array}{l}\text { Depth }^{1} \\
\text { (cm bs) }\end{array}$ & $\begin{array}{c}\text { Phosphate } \\
\text { (ppb) }\end{array}$ & $\begin{array}{l}\text { Fish Bones } \\
\text { (NISP) }\end{array}$ & $\begin{array}{c}\text { Vol. }<2 \mathrm{~mm} \\
(\mathrm{~mL})\end{array}$ \\
\hline ElTb-10 & McNaughton & 4500 & A22 & 238 & 5264 & 41 & 325 \\
\hline ElTb-10 & McNaughton & 4500 & A23 & 244 & 5529 & 13 & 450 \\
\hline ElTb-10 & McNaughton & 4500 & A24 & 256 & 6424 & 29 & 450 \\
\hline ElTb-10 & McNaughton & 4500 & A25 & 266 & 3920 & 12 & 250 \\
\hline ElTb-10 & McNaughton & 4500 & A26 & 274 & 3512 & 4 & 325 \\
\hline ElTb-10 & McNaughton & 4500 & $\mathrm{~A} 27$ & 284 & 3882 & 24 & 450 \\
\hline ElTb-10 & McNaughton & 4500 & A28 & 294 & 3046 & 38 & 425 \\
\hline ElTb-10 & McNaughton & 4500 & A29 & 302 & 3765 & 23 & 425 \\
\hline ElTb-10 & McNaughton & 4500 & A30 & 310 & 3282 & 40 & 350 \\
\hline ElTb-10 & McNaughton & 4500 & A31 & 316 & 2554 & 21 & 450 \\
\hline ElTb-10 & McNaughton & 4500 & A32 & 320 & 2702 & 33 & 575 \\
\hline ElTb-10 & McNaughton & 4500 & A33 & 330 & 2067 & 8 & 650 \\
\hline ElTb-10 & McNaughton & 4500 & AVERAGE & --- & 5178 & --- & --- \\
\hline EkSt-1 & Katit & 4260 & $\mathrm{C} 1$ & 25 & 11748 & 7 & 388 \\
\hline EkSt-1 & Katit & 4260 & $\mathrm{C} 2$ & 45 & 10866 & 24 & 267 \\
\hline EkSt-1 & Katit & 4260 & C3 & 74 & 11407 & 66 & 416 \\
\hline EkSt-1 & Katit & 4260 & C4 & 93 & 10482 & 43 & 260 \\
\hline EkSt-1 & Katit & 4260 & $\mathrm{C} 5$ & 112 & 10930 & 152 & 362 \\
\hline EkSt-1 & Katit & 4260 & C6 & 124 & 10491 & 122 & 318 \\
\hline EkSt-1 & Katit & 4260 & $\mathrm{C7}$ & 140 & 12434 & 99 & 359 \\
\hline EkSt-1 & Katit & 4260 & C8 & 152 & 9458 & 90 & 424 \\
\hline EkSt-1 & Katit & 4260 & C9 & 166 & 12492 & 41 & 390 \\
\hline EkSt-1 & Katit & 4260 & C10 & 178 & 10640 & 57 & 389 \\
\hline EkSt-1 & Katit & 4260 & C11 & 184 & 14839 & 19 & 339 \\
\hline EkSt-1 & Katit & 4260 & $\mathrm{C} 12$ & 194 & 11008 & 24 & 304 \\
\hline EkSt-1 & Katit & 4260 & $\mathrm{C} 13$ & 204 & 11744 & 13 & 851 \\
\hline EkSt-1 & Katit & 4260 & AVERAGE & --- & 11426 & --- & --- \\
\hline EjSw-1 & Cockmi & 8590 & $\mathrm{~A} 2$ & 22 & 2858 & 12 & 266 \\
\hline EjSw-1 & Cockmi & 8590 & A4 & 70 & 1750 & 6 & 231 \\
\hline EjSw-1 & Cockmi & 8590 & A6 & 92 & 3449 & 11 & 304 \\
\hline EjSw-1 & Cockmi & 8590 & A8 & 115 & 3634 & 23 & 348 \\
\hline EjSw-1 & Cockmi & 8590 & A10 & 144 & 2982 & 14 & 244 \\
\hline EjSw-1 & Cockmi & 8590 & A12 & 176 & 3134 & 16 & 271 \\
\hline EjSw-1 & Cockmi & 8590 & A14 & 188 & 5064 & 54 & 282 \\
\hline EjSw-1 & Cockmi & 8590 & A16 & 231 & 4540 & 26 & 233 \\
\hline EjSw-1 & Cockmi & 8590 & A18 & 255 & 4162 & 20 & 231 \\
\hline EjSw-1 & Cockmi & 8590 & A20 & 282 & 5101 & 15 & 348 \\
\hline EjSw-1 & Cockmi & 8590 & A22 & 305 & 7467 & 17 & 346 \\
\hline EjSw-1 & Cockmi & 8590 & A24 & 319 & 6012 & 25 & 355 \\
\hline EjSw-1 & Cockmi & 8590 & A26 & 346 & 4087 & 26 & 344 \\
\hline EjSw-1 & Cockmi & 8590 & A28 & 372 & 2694 & 9 & 356 \\
\hline EjSw-1 & Cockmi & 8590 & A30 & 384 & 2555 & 9 & 313 \\
\hline EjSw-1 & Cockmi & 8590 & A32 & 404 & 1442 & 2 & 277 \\
\hline EjSw-1 & Cockmi & 8590 & A34 & 416 & 2408 & 0 & 165 \\
\hline EjSw-1 & Cockmi & 8590 & AVERAGE & --- & 3726 & --- & --- \\
\hline
\end{tabular}


M.A. Thesis - K. Carter; McMaster University - Anthropology

\begin{tabular}{|c|c|c|c|c|c|c|c|}
\hline Site & Site Name/Location & $\begin{array}{c}\text { Site Area } \\
\left(\mathrm{m}^{2}\right)\end{array}$ & Auger & $\begin{array}{l}\text { Depth }^{1} \\
\text { (cm bs) }\end{array}$ & $\begin{array}{c}\text { Phosphate } \\
\text { (ppb) }\end{array}$ & $\begin{array}{c}\text { Fish Bones } \\
\text { (NISP) }\end{array}$ & $\begin{array}{c}\text { Vol. }<2 \mathrm{~mm} \\
(\mathrm{~mL})\end{array}$ \\
\hline$\overline{E j S v-5}$ & Darby Channel & 540 & $A 2$ & 68 & 1501 & 3 & 556 \\
\hline EjSv-5 & Darby Channel & 530 & A4 & 94 & 1797 & 44 & 356 \\
\hline EjSv-5 & Darby Channel & 530 & A6 & 107 & 1380 & 9 & 323 \\
\hline EjSv-5 & Darby Channel & 530 & A8 & 124 & 1645 & 3 & 367 \\
\hline EjSv-5 & Darby Channel & 530 & A10 & 137 & 1399 & 6 & 282 \\
\hline EjSv-5 & Darby Channel & 530 & A12 & 153 & 1375 & 35 & 334 \\
\hline EjSv-5 & Darby Channel & 530 & A14 & 164 & 1226 & 34 & 371 \\
\hline EjSv-5 & Darby Channel & 530 & A16 & 178 & 1124 & 28 & 438 \\
\hline EjSv-5 & Darby Channel & 530 & A18 & 191 & 1090 & 17 & 332 \\
\hline EjSv-5 & Darby Channel & 530 & A20 & 209 & 1689 & 4 & 196 \\
\hline EjSv-5 & Darby Channel & 530 & AVERAGE & --- & 1423 & $-\cdots-$ & $-\cdots-$ \\
\hline EjSv-9 & Taylor Bay & 2040 & A2 & 37 & 852 & 7 & 415 \\
\hline EjSv-9 & Taylor Bay & 2040 & A4 & 81 & 2931 & 27 & 326 \\
\hline EjSv-9 & Taylor Bay & 2040 & A6 & 114 & 4051 & 29 & 329 \\
\hline EjSv-9 & Taylor Bay & 2040 & A8 & 138 & 4751 & 39 & 326 \\
\hline EjSv-9 & Taylor Bay & 2040 & A10 & 173 & 3347 & 9 & 296 \\
\hline EjSv-9 & Taylor Bay & 2040 & A12 & 221 & 3377 & 25 & 286 \\
\hline EjSv-9 & Taylor Bay & 2040 & AVERAGE & --- & 3218 & --- & --- \\
\hline EjSv-10 & Taylor Bay & 1300 & D1 & 84 & 2002 & 11 & 510 \\
\hline EjSv-10 & Taylor Bay & 1300 & D3 & 104 & 3695 & 8 & 304 \\
\hline EjSv-10 & Taylor Bay & 1300 & D5 & 127 & 4659 & 15 & 369 \\
\hline EjSv-10 & Taylor Bay & 1300 & D7 & 150 & 5212 & 5 & 342 \\
\hline EjSv-10 & Taylor Bay & 1300 & D9 & 178 & 4232 & 10 & 318 \\
\hline EjSv-10 & Taylor Bay & 1300 & D11 & 206 & 4385 & 13 & 345 \\
\hline EjSv-10 & Taylor Bay & 1300 & D13 & 229 & 2579 & 7 & 279 \\
\hline EjSv-10 & Taylor Bay & 1300 & D15 & 254 & 2055 & 3 & 409 \\
\hline EjSv-10 & Taylor Bay & 1300 & D17 & 268 & 2010 & 3 & 207 \\
\hline EjSv-10 & Taylor Bay & 1300 & AVERAGE & --- & 3425 & --- & --- \\
\hline \multicolumn{8}{|c|}{$\begin{array}{l}{ }^{1} \text { Depths are the top of each sample. E.g. EjSv-10, D1 is } 84-103 \mathrm{~cm} \text { below surface. } \\
\text { samples from Namu are actually from Auger E; fish bones from Auger A were not } \\
\text { ger A was chosen for phosphate analysis because it was the only auger where fine } \\
\text { natrix was retained for analysis. Auger E is located in proximity to Auger A and fish } \\
\text { bones are considered representative. }\end{array}$} \\
\hline
\end{tabular}

